Benchmark Data for a

Large Reprocessing Plant

for Evaluation of Advanced

Data Analysis Algorithms

and Safeguards System Design

Tom L. Burr

C. Alton Coulter

Larry E. Wangen

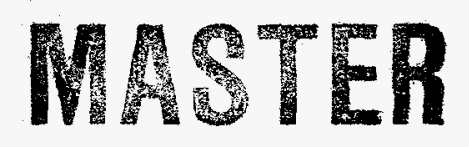

DISTARUTION OF THS DOCUMENT IS UNIMTED 


\section{DISCLAIMER}

This report was prepared as an account of work sponsored by an agency of the linited States Government. Neither the United States Government nor any agency thereof, nor any of their employees, make any warranty, express or implied, or assumes any legal liability or responsibility for the accuracy, completeness, or usefulness of any information, apparatus, product, or process disclosed, or represents that its use would not infringe privately owned rights. Reference herein to any specific commercial product, process, or service by trade name, trademark, manufacturer, or otherwise does not necessarily constitute or imply its endorsement, recommendation, or favoring by the United States Goverument or any agency thereof. The views and opinions of authors expressed herein do not necessarily state or reflect those of the United States Government or any agency thereof. 


\section{DISCLAIMER}

Portions of this document may be illegible electronic image products. Images are produced from the best available original document. 


\section{Contents}

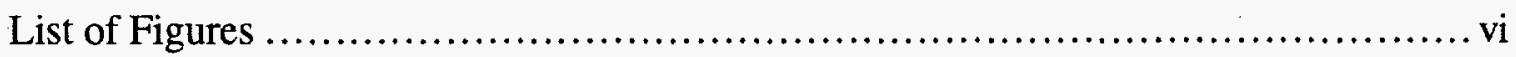

List of Tables..................................................................... vii

Abstract ....................................................................... 1

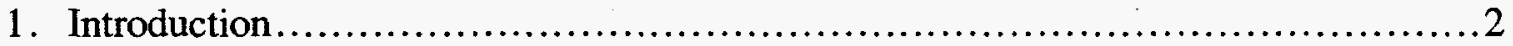

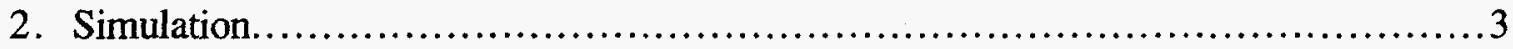

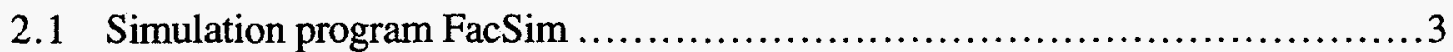

2.2 Adding measurement uncertainties to the simulated results .....................4

2.3 Diversion scenarios studies .............................................. 4

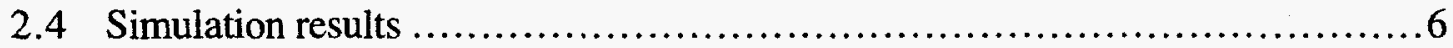

2.5 Implications for detectability of diversion strategies ..................... 32

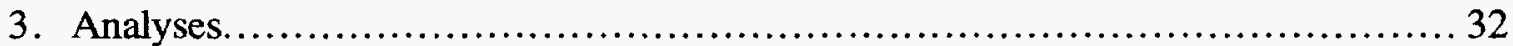

3.1 General approach to the analyses...................................... 32

3.2 Data expected ........................................................ 33

3.3 Measurement error modeling........................................... 34

3.4 Estimated loss detection probabilities .................................... 35

3.4.1 Three-tank system, $V_{8 \mathrm{~kg}}$ and $\mathrm{M}_{8 \mathrm{~kg}}$ cases......................... 36

3.4.1.1 Protracted loss using SM data .......................... 37

3.4.1.2 Abrupt loss using SM data............................. 38

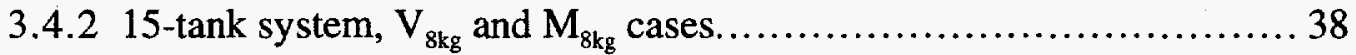

3.4.2.1 Protracted loss using SM data ............................... 38

3.4.2.2 Abrupt loss using SM data............................... 40

3.4.3 15-tank system, abrupt removal of less than $V_{8 \mathrm{~kg}}$ or $\mathrm{M}_{8 \mathrm{~kg}}$ cases........ 40

3.5 Analyses: summary and conclusions................................... 42

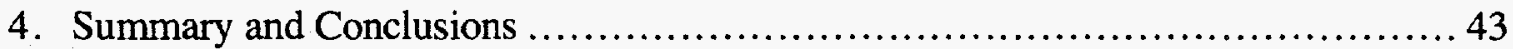

References................................................................ 43

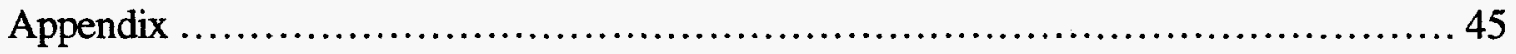




\section{List of Figures}

Fig. 1. Case 1a. 0.83-liter (0.1\%) diversion without replacement from the output accountability vessel $1441 \mathrm{~V} 70$ when the volume was nominally static...............7

Fig. 2. Case 1c. 2.49-liter (0.3\%) diversion without replacement from the output accountability vessel $1441 \mathrm{~V} 70$ when the volume was nominally static...............8

Fig. 3. Case 1e. 2.4-liter (0.3\%) diversion without replacement from control vessel 1421 V73 when the volume was nominally static

Fig. 4. Case 1f. 8.3-liter (1.0\%) diversion without replacement from the output accountability vessel $1441 \mathrm{~V} 70$ when the volume was nominally static.............. 10

Fig. 5. Case 1h. 8.0-liter (1.0\%) diversion without replacement from control vessel 1421 V73 when the volume was nominally static.............................. 11

Fig. 6. Case 1i. 41.5-liter (5.0\%) diversion without replacement from the output accountability vessel $1441 \mathrm{~V} 70$ when the volume was nominally static............. 12

Fig. 7. Case 1k. 125 -liter (5.0\%) diversion without replacement from control vessel 1211 V55 when the volume was nominally static.............................. 13

Fig. 8. Case 2a. 0.83-liter $(0.1 \%)$ diversion with replacement from the output accountability vessel $1441 \mathrm{~V} 70$ when the volume was nominally static.............. 14

Fig. 9. Case 2c. 2.49-liter (0.3\%) diversion with replacement from the output accountability vessel $1441 \mathrm{~V} 70$ when the volume was nominally static.............. 15

Fig. 10. Case 2d.4-liter (0.3\%) diversion with replacement from control vessel 1421 V73 when the volume was nominally static............................... 16

Fig. 11. Case 2e. 8.3-liter (1.0\%) diversion with replacement from output accountability vessel $1441 \mathrm{~V} 70$ when the volume was nominally static............. 17

Fig. 12. Case 2f. 25 -liter (1.0\%) diversion with replacement from control vessel $1211 \mathrm{~V} 55$ when the volume was nominally static.............................. 18

Fig. 13. Case 2g. 41.5-liter (5.0\%) diversion with replacement from output accountability vessel $1441 \mathrm{~V} 70$ when the volume was nominally static.............. 19

Fig. 14. Case 2h. 40-liter (1.0\%) diversion with replacement from buffer vessel 1421 V71 when the volume was nominally static............................ 20

Fig. 15. Case 3a-1 . 0.83-liter (0.1\%) diversion without replacement from output accountability vessel 1441 V70 during an input transfer.......................... 21

Fig. 16. Case 3a-2. 0.83-liter $(0.1 \%)$ diversion without replacement from output accountability vessel 1441 V70 during an input transfer........................... 22

Fig. 17. Case 3b-1. 2.49-liter (0.3\%) diversion without replacement from output accountability vessel 1441 V70 during an input transfer.......................... 23

Fig. 18. Case 3c-2. 8.0-liter (1.0\%) diversion without replacement from buffer vessel 1421 V71 during an output transfer. ............................................. 24

Fig. 19. Case 3d-2. 125-liter (5.0\%) diversion without replacement from control vessel

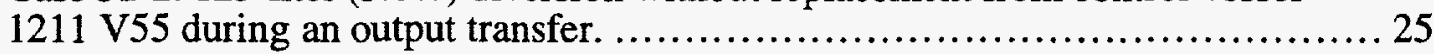

Fig. 20. Case 4a-1. 0.83-liter (0.1\%) diversion with replacement from output accountability vessel 1441 V70 during an input transfer.......................... 26

Fig. 21. Case 4b-1. 2.49-liter (0.3\%) diversion with replacement from output accountability vessel 1441 V70 during an input transfer......................... 27

Fig. 22. Case 4c-1. 8.0-liter (1.0\%) diversion with replacement from control vessel 1421 V73 during an output transfer. ......................................... 28

Fig. 22. Case 4d-a. 981-liter (5.0\%) diversion with replacement from receiving vessel 1211 V10 during an output transfer............................................... 29

Fig. 24. Case $4 \mathrm{~d}-2 \mathrm{~b}$. Volume plots for vessels 1211 V 10 and 1211 V11 during the diversion with replacement defined in Figure 23.............................. 30

Fig. 25. Case 4d-2c. Plutonium inventory plots for vessels 1211 R20 and 1211 R30 following the diversion with replacement shown in Figure $23 \ldots \ldots \ldots \ldots \ldots \ldots \ldots . \ldots . \ldots 31$

Fig. 26. Approximately 4 days of level readings at $\sim 4$ minute intervals for the first 9 tanks.. 33 


\section{List of Tables}

Table 1. Diversion scenarios simulated in this study ..................................5

Table 2. Tanks $1-3, B C$ results given for $n=0.1^{*}$ nships, $n=0.2^{*}$ nships,

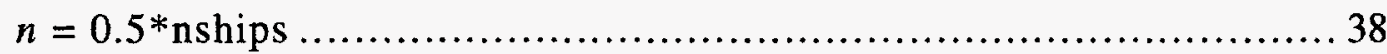

Table 3. Tanks $1-15, \sigma_{\mathrm{R}}=0.1 \%$ relative, $\mathrm{BC}$ results for $n=0.1{ }^{*}$ nships, $0.2 *$ nships, $0.5^{*}$ nships............................................. 39

Table 4. Tanks $1-15, \sigma_{\mathrm{R}}=0.2 \%$ relative, $\mathrm{BC}$ results for $n=0.1^{*} \mathrm{nships}$, $0.2 *$ nships, $0.5 *$ nships

Table 5. Tanks $1-15, \sigma_{\mathrm{R}}=0.5 \%$ relative, $\mathrm{BC}$ results for $n=0.1^{*}$ nships, $0.2 *$ nships, $0.5 *$ nships 39

Table 6. Abrupt volume loss, $\sigma_{\mathrm{R}}=\sigma_{\mathrm{S}}=0.1 \%$ during wait modes ..................... 40

Table 7. Abrupt mass loss, $\sigma_{\mathrm{R}}=\sigma_{\mathrm{S}}=0.1 \%$ during wait modes ........................ 41

Table 8. Abrupt volume loss, $\sigma_{\mathrm{R}}=\sigma_{\mathrm{S}}=0.1 \%$ during transfer modes $\ldots \ldots \ldots \ldots \ldots \ldots \ldots \ldots . \ldots \ldots$

Table 9. Abrupt mass loss, $\sigma_{\mathrm{R}}=\sigma_{\mathrm{S}}=0.1 \%$ during transfer modes................... 41

Table 10. Abrupt volume loss, $\sigma_{\mathrm{R}}=\sigma_{\mathrm{S}}=0.3 \%$ during transfer modes..................41

Table 11. Abrupt mass loss, $\sigma_{\mathrm{R}}=\sigma_{\mathrm{S}}=0.3 \%$ during transfer modes $\ldots \ldots \ldots \ldots \ldots \ldots \ldots \ldots . \ldots 2$

Table 12. Loss detection probabilities....................................... 42 


\title{
Benchmark Data for a Large Reprocessing Plant for Evaluation of Advanced Data Analysis Algorithms and Safeguards System Design
}

\author{
by \\ Tom L. Burr, C. Alton Coulter, and Larry E. Wangen
}

\begin{abstract}
This report describes the simulation and analysis of solution level and density (L,D) in all key main process tanks in a large reprocessing plant. In addition, initial provisions were made to include temperature $(\mathrm{T})$ data in the analysis at a later time. FacSim, a simulation program developed at Los Alamos, was used to generate simulated process operating data for the Rokkasho Reprocessing Plant (RRP) that is now under construction in Japan. Both normal facility operation and more than thirty abruptdiversion scenarios were modeled over 25-day periods of simulated operation beginning with clean startup of the facility. The simulation tracked uranium, plutonium (both +3 and +4 oxidation states), $\mathrm{HNO}_{3}$, diluent, and tributyl phosphate from the input accountability vessel to the plutonium output accountability vessel, with the status of each process vessel and many pipes recorded at intervals of approximately four minutes. These data were used to determine solution volume and density values in each process vessel as a function of time.

For the analysis portion of the task, specialized code developed at Los Alamos was used. This code first finds and classifies all key tank events so that each section of each tank's history can be identified as either a wait or a transfer mode. The key analyses then involve using historical data and/or measurement error models to initially label each mode as normal or abnormal. All initially labeled abnormal modes (such as a large-volume shipper-receiver difference during a tank-to-tank transfer) are considered to be abrupt anomalies. All initially labeled normal modes are further scrutinized in aggregate over time to monitor for protracted anomalies, such as trickle losses. We apply traditional statistical decision theory in conjunction with measurement error models to detect both abrupt and protracted anomalies. Our measurement error models suggest that a large fraction of the total measurement error will involve the systematic errors related to the volume measurements. Therefore, we show the possibility of
\end{abstract}


reducing the effect of systematic volume errors by using solution monitoring (SM) data to calculate bias corrections. We divide the effort into 4 activities for a 3-tank system and for a more realistic 15-tank system. We say a tank is in wait mode if its level change is due only to measurement error. Otherwise, a tank is in transfer mode. The 4 activities are (1) monitor each tank for volume loss during each wait mode, (2) monitor each tank for mass loss during each wait mode, (3) monitor each tank for volume loss during each transfer mode, and (4) monitor each tank for mass loss during each transfer mode. The success of this effort will depend largely on the performance of our proposed bias corrections to the volume measurements. To apply bias corrections, we require that some reasonable number of transfers (for example, 20) is known to have no true loss. The effectiveness of the technique will depend on the relative sizes of the random and systematic errors involved because the main outcome is a reduction of the systematic error variances. If there are large variations in true (legitimate) temporary losses such as pipe holdup that would add to the random error variance in our model, then the effectiveness will be reduced. Even in such cases we will show that there can be improved protracted loss detection, and there will definitely be improved abrupt loss detection. Though we can easily show the benefits of using SM data for analyses similar to those presented here, we acknowledge that because of the large throughput expected at RRP, even the most optimistic estimates suggest that protracted loss detection for a trickle diversion will not meet the detection goal.

\section{Introduction}

The purposes of this task were

1. to use simulation to generate benchmark data sets representing the time-dependent operating parameters of a large reprocessing plant under both nominal and anomalous operating conditions, and

2. to use the benchmark data sets to test various data analysis methods being explored under Member State Support Programmes.

The simulations were performed with an enhanced version of the program FacSim developed at Los Alamos National Laboratory. Through the courtesy of Japan Nuclear Fuel Limited* (JNFL) it was possible to use the original design of the Rokkasho Reprocessing Plant (RRP) as the basis for the simulations. Analyses of the simulation results were performed at Los Alamos National

\footnotetext{
*See the Appendix, which contains a copy of a letter from Mr. Itsuo Kadokura, Manager of the Safeguards Planning Division, Safeguards Department, of JNFL. 
Laboratory and at the University of Glasgow. This report describes the simulations and the data analyses performed at Los Alamos National Laboratory.

\section{Simulation}

\subsection{Simulation program FacSim}

The simulation program FacSim was previously used to simulate operation of RRP for the purpose of determining material throughputs and in-process inventories. A description of FacSim and of the simulation results are given in the report "Near-Real-Time Accounting (NRTA) as a Safeguards Measure at the Rokkasho Reprocessing Plant" [1]. Because a copy of this report has been provided to the International Atomic Energy Agency (IAEA) by JNFL, we shall not repeat the complete description of FacSim here.

However, in the previous simulations only uranium and plutonium amounts and solution volumes were calculated. Because the analysis methods tested in the current task examine both solution volumes and solution densities, it was necessary to expand the set of solution components tracked in the simulation beyond this minimal set. The solution components used in the current study were the following:

Aqueous: $\quad \mathrm{H}_{2} \mathrm{O}, \mathrm{HNO}_{3}, \mathrm{Pu}^{\mathrm{III}}, \mathrm{Pu}^{\mathrm{Iv}}, \mathrm{U}$;

Organic: diluent, TBP, $\mathrm{HNO}_{3}, \mathrm{Pu}^{\mathrm{iv}}, \mathrm{U}$, where $\mathrm{Pu}^{\mathrm{III}}$ and $\mathrm{Pu}^{\mathrm{IV}}$ designate the +3 and +4 oxidation states of plutonium. Empirical expressions for the densities of the aqueous and organic solutions as functions of the quantities of these constituents were developed by beginning with the density expressions given by W. S. Groenier in the Technical Manual for SEPHIS MOD4, Version 2.11 [2]. The numerical coefficients in the expressions given by Groenier were then adjusted somewhat to obtain better agreement with the solution densities specified in the RRP flowsheets. The resulting expressions for aqueous and organic solution densities are probably accurate only to a few percent, but variations in the calculated densities due to changes in solution constituents are of the correct size to indicate the effectiveness of analysis methods in detecting anomalous changes in density.

The initial work plan anticipated that solution temperatures would be incorporated into the calculation, but for several reasons-including lack of required process information-this was not done.

In pulsed columns there is some transfer of nitrate ions $\left(\mathrm{HNO}_{3}^{-}\right)$between the aqueous and organic phases independently of the transfer of nitrate ions complexed with $\mathrm{U}$ and $\mathrm{Pu}$. This noncomplexed nitrate ion transfer was neglected because the representation of pulsed columns used by FacSim does not contain enough detail to allow its calculation. This omission has no significant effect on the detectability of the material diversions that were simulated. 


\subsection{Adding measurement uncertainties to the simulated results}

Measurements of solution levels and densities in reprocessing plants are subject to errors from a number of sources, and data analysis algorithms attempt to detect anomalies that may be partially masked by the effect of these errors. The volume/level and density values calculated by FacSim do not, of course, contain these errors. It was decided that for the purposes of the present task the FacSim values should be recorded as calculated rather than having noise added to the values after the fact, and measurement error would be added to the values calculated by FacSim in the process of performing data analysis. This is a more flexible approach, because an analysis algorithm can be tested repeatedly on the same simulation data set with different amounts of measurement error added to the simulated values on successive trials.

\subsection{Diversion scenarios studied}

All simulations covered a period of 24 days beginning with clean startup. For the last 11 days of simulated operation, when the facility was essentially at "steady state," process vessel and pipe contents were recorded at intervals of 3.75 minutes ( $1 / 16$ hour). The four following different types of "abrupt" diversions were considered:

1. diversion without replacement from a tank with static volume,

2. diversion with replacement from a tank with static volume,

3. diversion without replacement from a tank during a solution transfer, and

4. diversion with replacement from a tank during a solution transfer.

Twenty-seven diversion scenarios were considered, with eight of these simulated twice using different diversion initiation times for a total of 35 cases. These 35 cases are

- 11 diversions of type 1 , which we shall designate by $1 \mathrm{a}, 1 \mathrm{~b}, \ldots, 1 \mathrm{k}$;

- 8 diversion $s$ of type 2 , which we shall designate by $2 \mathrm{a}, \ldots, 2 \mathrm{~h}$;

- 4 diversions of type 3 , each initiated with two different initial sets of random numbers, which we shall designate by $3 \mathrm{a}-1,3 \mathrm{a}-2, \ldots, 3 \mathrm{~d}-2$; and

- 4 diversions of type 4 , each initiated with two different sets of random numbers, which we shall designate by $4 \mathrm{a}-1,4 \mathrm{a}-2, \ldots, 4 \mathrm{~d}-2$.

In each case a single diversion occurred during the final eleven days of simulated operation at a time that was randomly selected within an appropriate time interval (during a period of static volume or during a transfer, as appropriate). In every case diverted material flowed to an undeclared tank through an undeclared pipe; and in the case of diversion with replacement, the makeup solution flowed from an undeclared source tank through an undeclared source pipe. The amount of solution diverted was calculated as a fraction of the tank's active volume, with the fraction ranging in size from $0.1 \%$ to $5.0 \%$. In most cases the diversion was assumed to occur from the output 
accountability tank 1441 V70, because this tank contains the most attractive material. However, diversions from other tanks were assumed as well, including a few diversions from tanks that precede the codecontamination cycle.

Note that the feeding and receiving vessels for the column cycles and the evaporator never have static volumes, so these vessels may be the most difficult locations in which to detect anomalies. Only one such vessel was considered in the diversion scenarios listed in Table 1 , and the parameters used for that scenario make the diversion unrealistically easy to identify.

Table 1. Diversion Scenarios Simulated in this Study.

\begin{tabular}{|c|c|c|c|}
\hline Case & $\begin{array}{l}\text { Diverted } \\
\text { Fraction }\end{array}$ & Tank & Action \\
\hline la & $0.1 \%$ & 1441 V70 Accountability Vessel & Diversion \\
\hline $1 \mathrm{~b}$ & $0.1 \%$ & 1441 V70 Accountability Vessel & Diversion \\
\hline $1 \mathrm{c}$ & $0.3 \%$ & 1441 V70 Accountability Vessel & Diversion \\
\hline $1 \mathrm{~d}$ & $0.3 \%$ & 1441 V70 Accountability Vessel & Diversion \\
\hline le & $0.3 \%$ & 1421 V73 Control Vessel & Diversion \\
\hline If & $1.0 \%$ & 1441 V70 Accountability Vessel & Diversion \\
\hline $1 \mathrm{~g}$ & $1.0 \%$ & 1441 V70 Accountability Vessel & Diversion \\
\hline $1 \mathrm{~h}$ & $1.0 \%$ & 1421 V73 Control Vessel & Diversion \\
\hline $1 \mathrm{i}$ & $5.0 \%$ & 1441 V70 Accountability Vessel & Diversion \\
\hline $1 \mathrm{j}$ & $5.0 \%$ & 1441 V70 Accountability Vessel & Diversion \\
\hline $1 \mathrm{k}$ & $5.0 \%$ & 1211 V55 Control Vessel & Diversion \\
\hline $2 a$ & $0.1 \%$ & 1441 V70 Accountability Vessel & Diversion with replacement \\
\hline $2 b$ & $0.1 \%$ & 1441 V70 Accountability Vessel & Diversion with replacement \\
\hline $2 c$ & $0.3 \%$ & 1441 V70 Accountability Vessel & Diversion with replacement \\
\hline $2 \mathrm{~d}$ & $0.3 \%$ & 1421 V73 Control Vessel & Diversion with replacement \\
\hline $2 \mathrm{e}$ & $1.0 \%$ & 1441 V70 Accountability Vessel & Diversion with replacement \\
\hline $2 \mathrm{f}$ & $1.0 \%$ & 1211 V55 Control Vessel & Diversion with replacement \\
\hline $2 \mathrm{~g}$ & $5.0 \%$ & 1441 V70 Accountability Vessel & Diversion with replacement \\
\hline $2 \mathrm{~h}$ & $5.0 \%$ & 1421 V71 Buffer Vessel & Diversion with replacement \\
\hline $3 a$ & $0.1 \%$ & 1441 V70 Accountability Vessel & Diversion during input transfer \\
\hline $3 \mathrm{~b}$ & $0.3 \%$ & 1441 V70 Accountability Vessel & Diversion during input transfer \\
\hline $3 c$ & $1.0 \%$ & 1421 V71 Buffer Vessel & Diversion during output transfer \\
\hline $3 \mathrm{~d}$ & $5.0 \%$ & 1211 V55 Control Vessel & Diversion during output transfer \\
\hline $4 \dot{a}$ & $0.1 \%$ & 1441 V70 Accountability Vessel & $\begin{array}{l}\text { Diversion with replacement } \\
\text { during input transfer }\end{array}$ \\
\hline $4 \mathrm{~b}$ & $0.3 \%$ & 1441 V70 Accountability Vessel & $\begin{array}{l}\text { Diversion with replacement } \\
\text { during input transfer }\end{array}$ \\
\hline $4 \mathrm{cc}$ & $1.0 \%$ & 1421 V73 Control Vessel & $\begin{array}{l}\text { Diversion with replacement } \\
\text { during output transfer }\end{array}$ \\
\hline $4 \mathrm{~d}$ & $5.0 \%$ & 1211 V10 Receiving Vessel & $\begin{array}{l}\text { Diversion with replacement } \\
\text { during output transfer }\end{array}$ \\
\hline
\end{tabular}




\subsection{Simulation results}

Results of the 35 simulated diversion cases (the 27 diversion scenarios shown in Table 1, with each of the last 8 scenarios simulated for two sets of initial random numbers) were written to 2 CD-ROMs. Results for cases 1a through $2 \mathrm{~h}$ are on the first CD-ROM, and those for cases 3a-1 through 4d-2 are on the second CD-ROM. The first CD-ROM also contains "case 0 " results for the facility under nominal operating conditions with no diversions. A copy of each of these CD-ROMs was sent to Dr. Tom Canada at the IAEA in February 1997. The root directory of each CD-ROM contains a file "DIVERSIONCASE.TXT" that contains the information shown in Table 1, and the simulation results for the various cases are in subdirectories labeled by the case numbers (case 1a, ..., case4d-2). Each subdirectory contains a file "Purexv.lst" that tells which file in the subdirectory holds the results for each of the facility process vessels and pipes. The tank into which material is diverted is always named "DIVERSION TANK 1," and by examining the output file for this tank one may determine the time at which the diversion occurred. The time immediately before that at which the diversion tank's contents first became nonzero is the time at which flow into the diversion tank began.

Because the complete set of output files on the two CD-ROMs contains nearly a gigabyte of text, and the output file for each process vessel contains nearly 400 kilobytes of text, it is not feasible to give complete tables of process vessel status parameters here. Instead, for a representative set of the diversion scenarios in Table 1 we give a series of figures showing volume and density information for a one- or two-hour period of simulated operation containing the diversion for the tank(s) directly affected by the diversion. The figure captions give the diversion parameters. In the majority of cases the effect of the diversion is masked in the figure by the nonzero line width of the graph. For this reason the approximate location of the beginning of the diversions are marked by a caret $\left({ }^{\wedge}\right)$ where needed.

When the diversion is not from the output accountability vessel, the information given in the figures can be used to identify the starting point for detecting changes in the contents of process vessels that are "downstream" from the vessel in which the diversion occurs. One can see these changes by examining the appropriate files on the CD-ROMs. An example of such downstream effects is given in Fig. 25, which shows the drop in the plutonium inventory of the 1211 R20 extraction column and the $1211 \mathrm{R} 30$ scrub column following the diversion-with-replacement from the 1211 V10 receiving vessel. The simulations whose results are contained on the CD-ROMs give a complete account of the effect of each diversion throughout the entire process and not just in the process vessel from which the diversion occurred.

\footnotetext{
- The indicated location is approximate because the graphing program used permits positioning characters, etc., only in minimum increments of 0.1 inch. As a result, the indicated beginning of the diversion on a volume plot is usually not at quite the same position as the indicated beginning of the same diversion on a density plot.
} 
1441 V70 Accountability Vessel

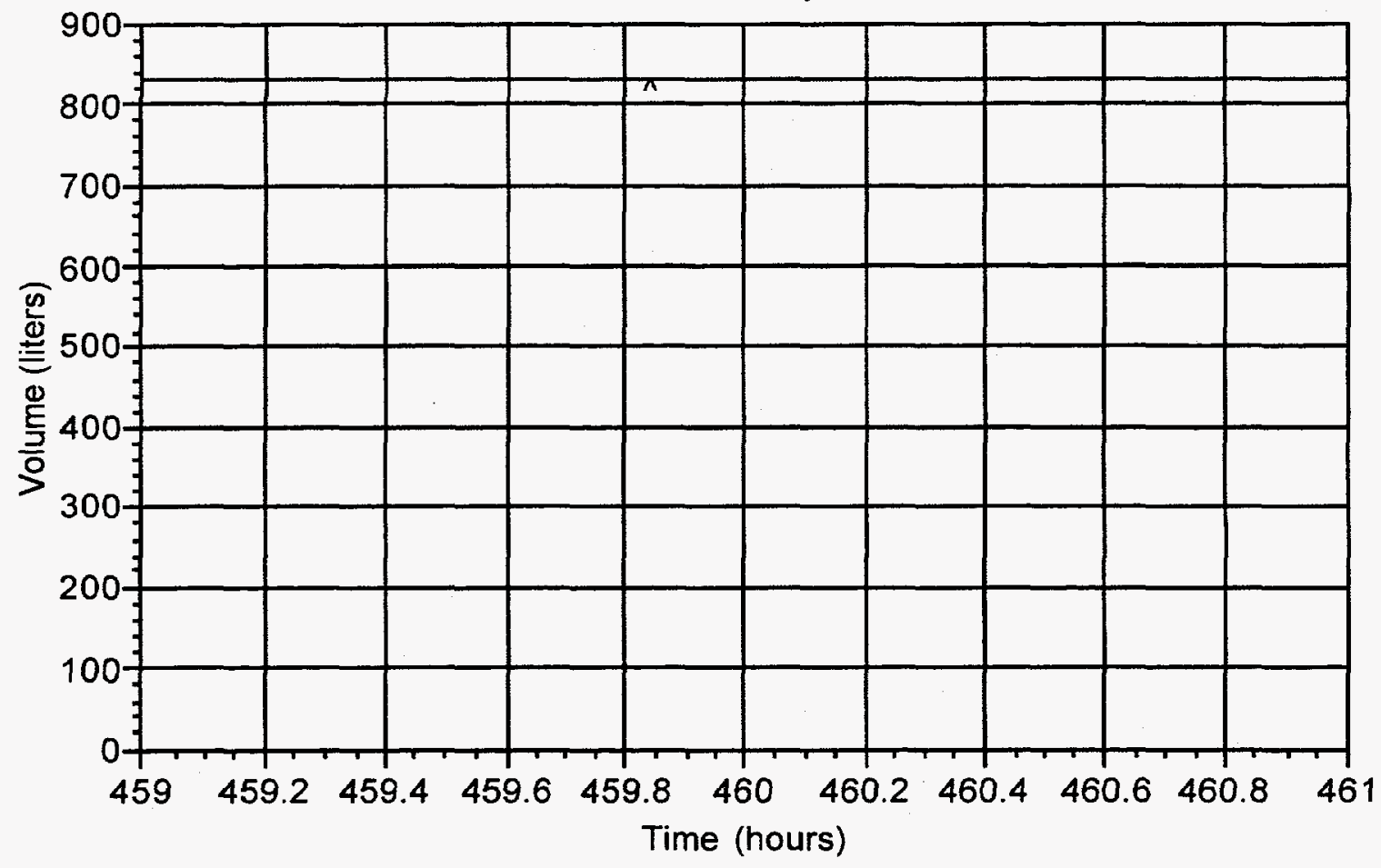

1441 V70 Accountability Vessel

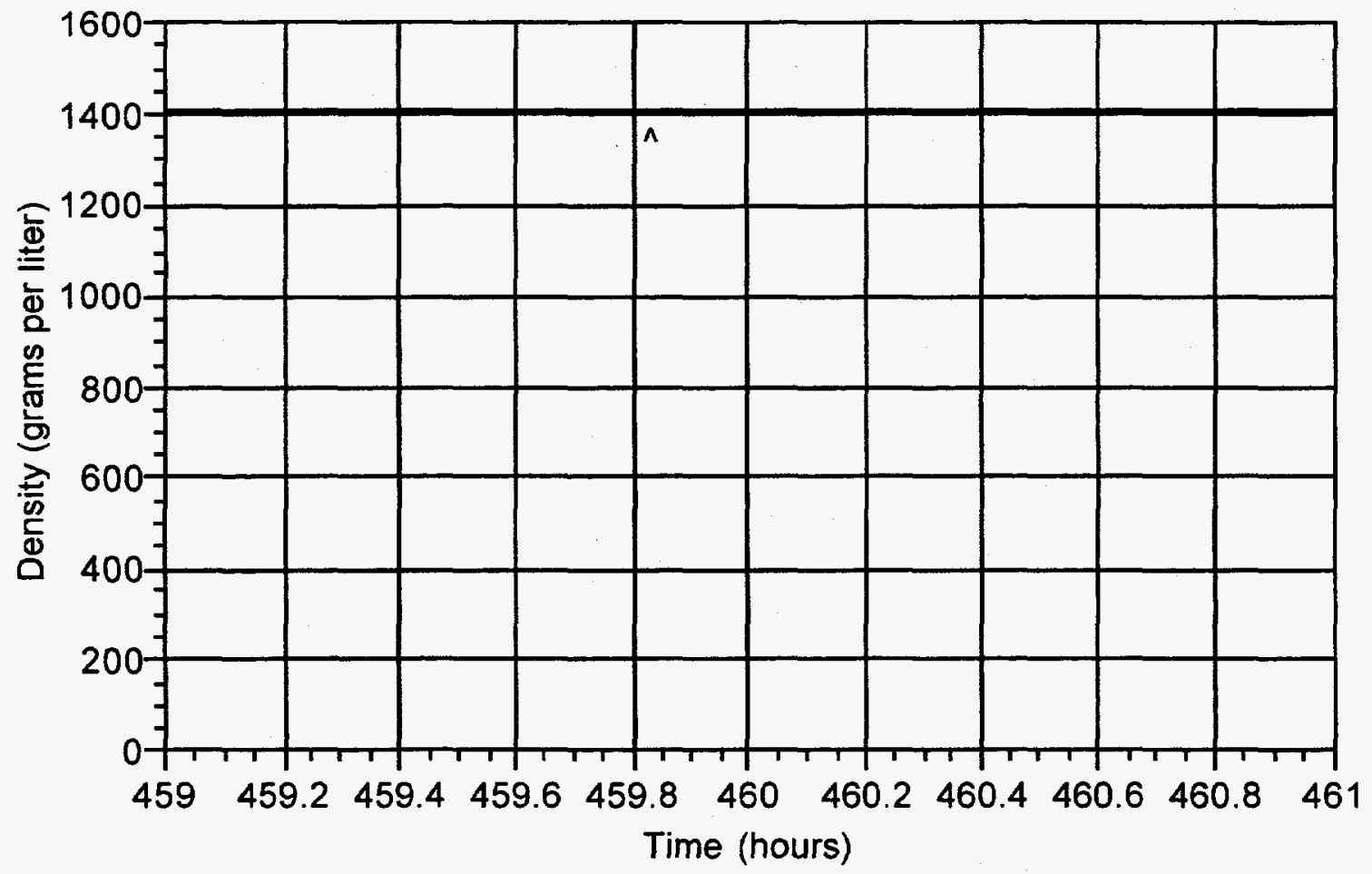

Fig. 1. Case 1a. 0.83-liter (0.1\%) diversion without replacement from the output accountability vessel 1441 V70 when the volume was nominally static. The diversion began at 459.8266 hours and lasted 5 minutes. 


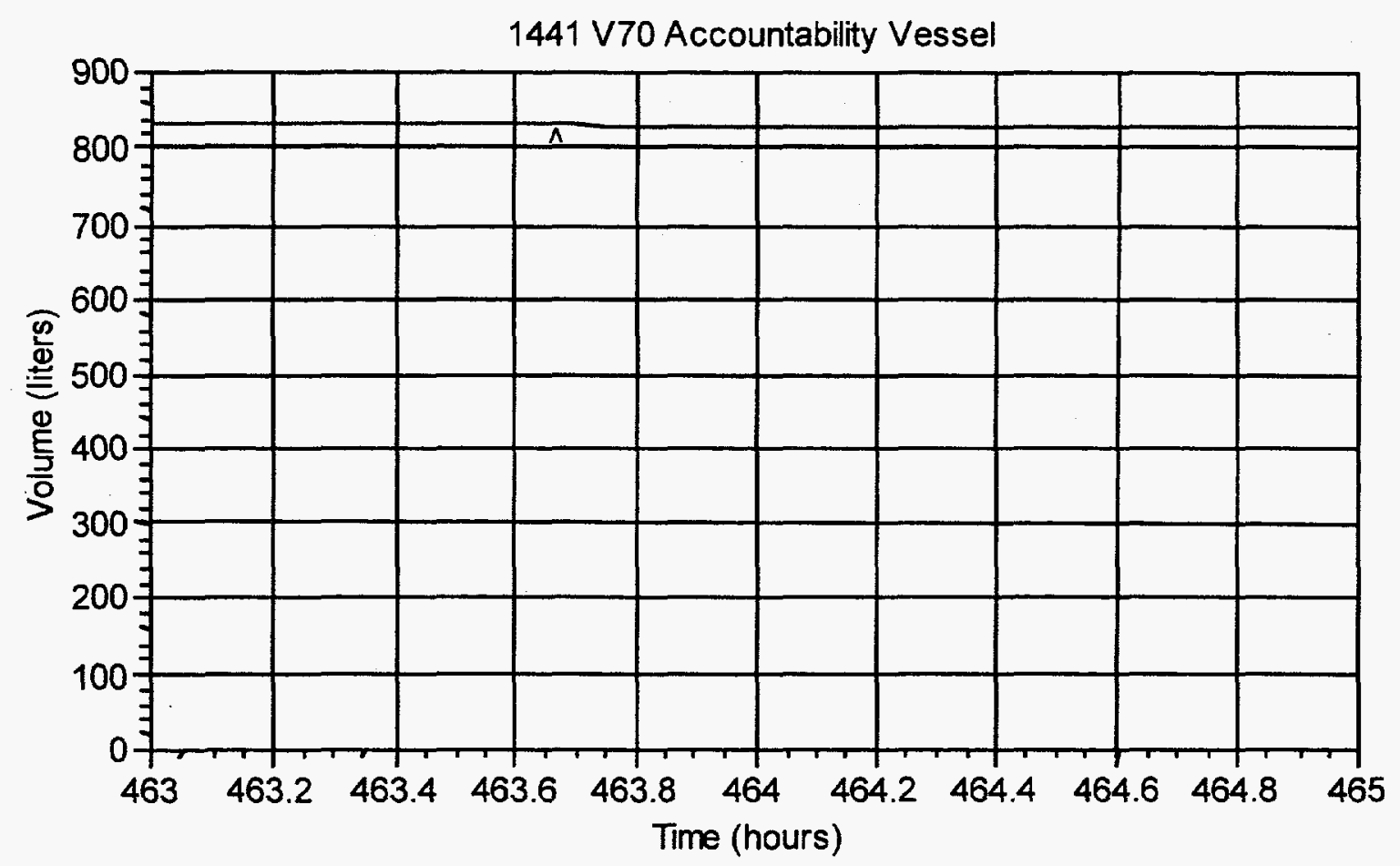

1441 V70 Accountability Vessel

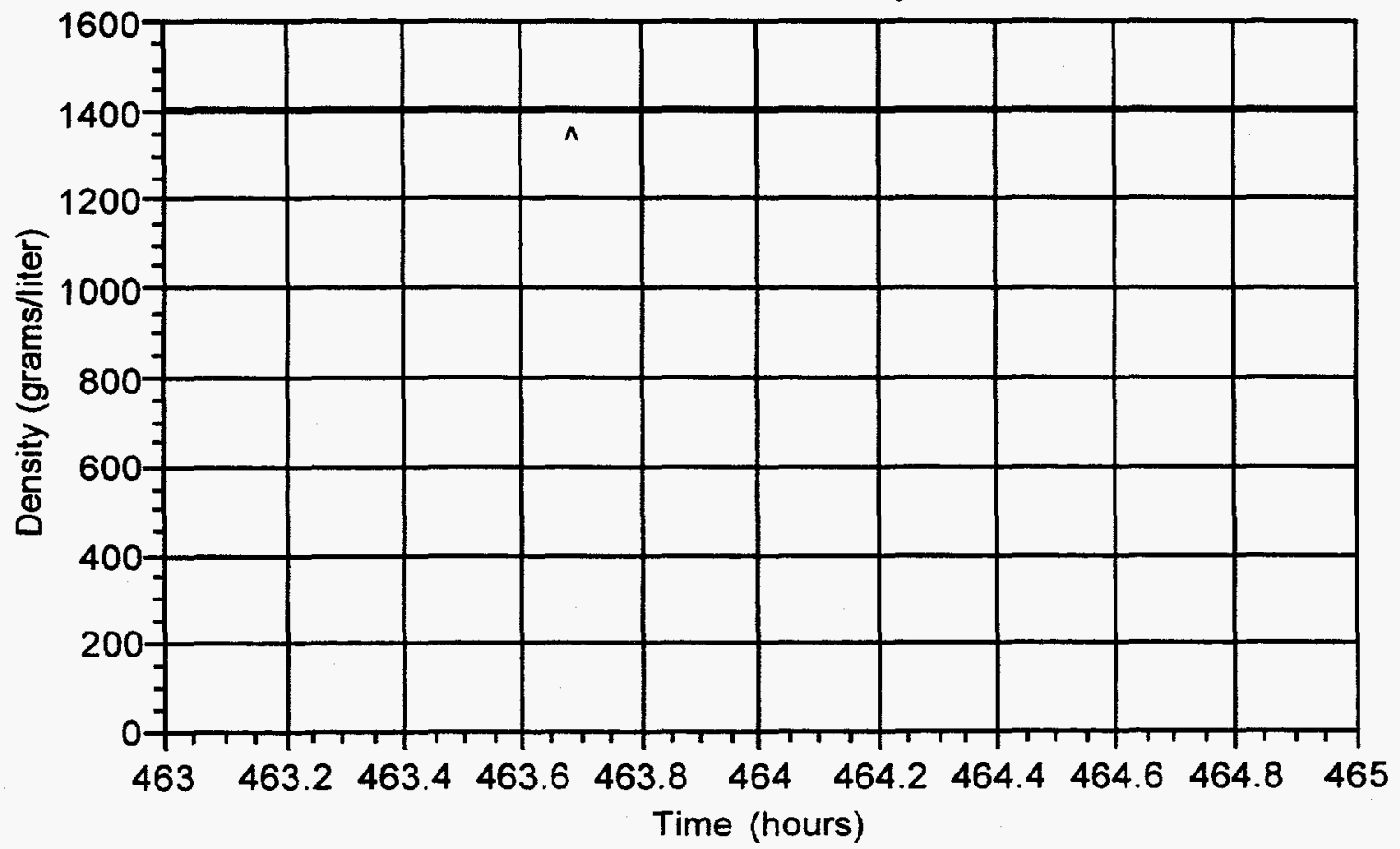

Fig. 2. Case 1c. 2.49-liter (0.3\%) diversion without replacement from the output accountability vessel 1441 V70 when the volume was nominally static. The diversion began at 463.6694 hours and lasted 5 minutes. 
1421 V73 Control Vessel

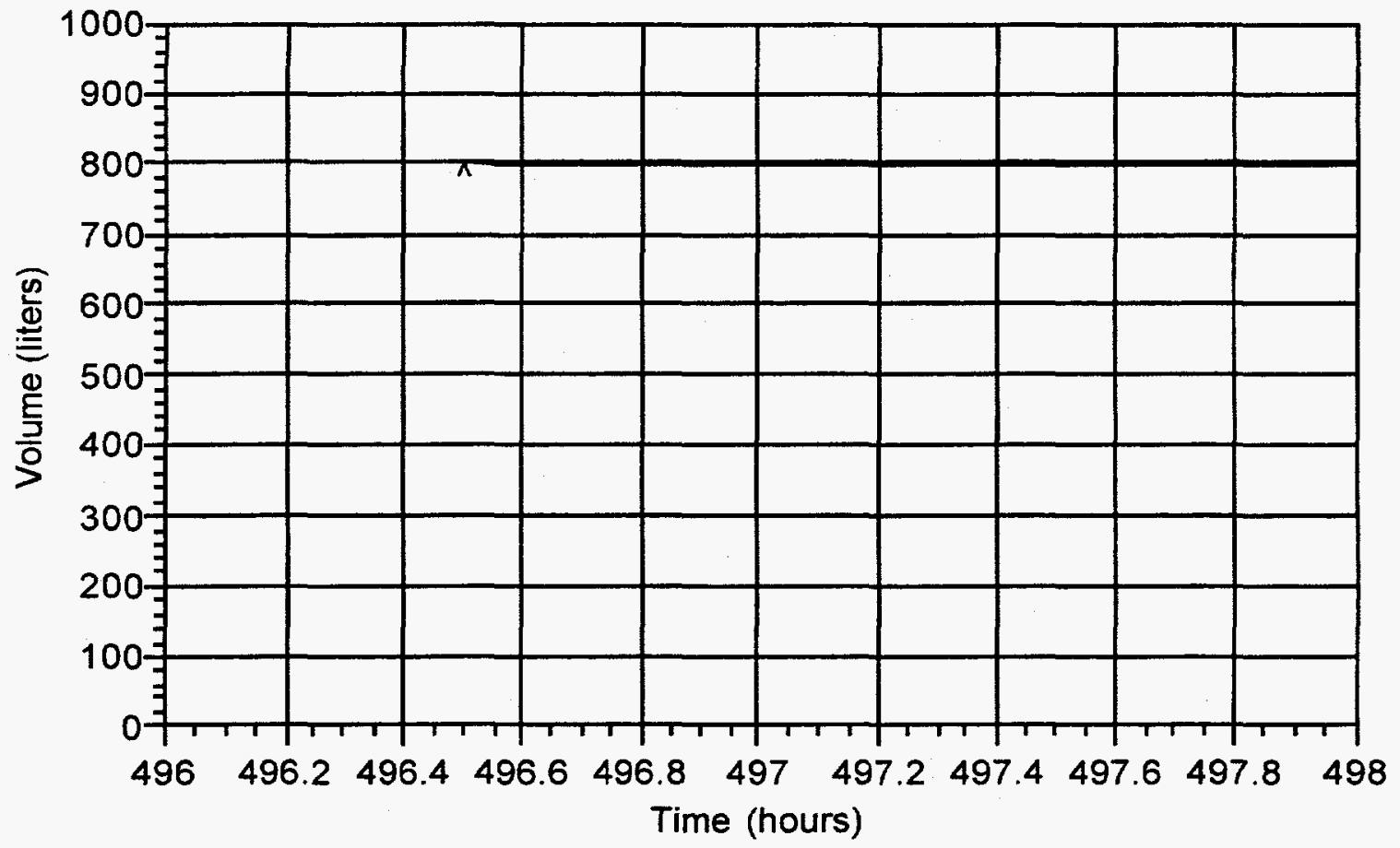

1421 V73 Control Vessel

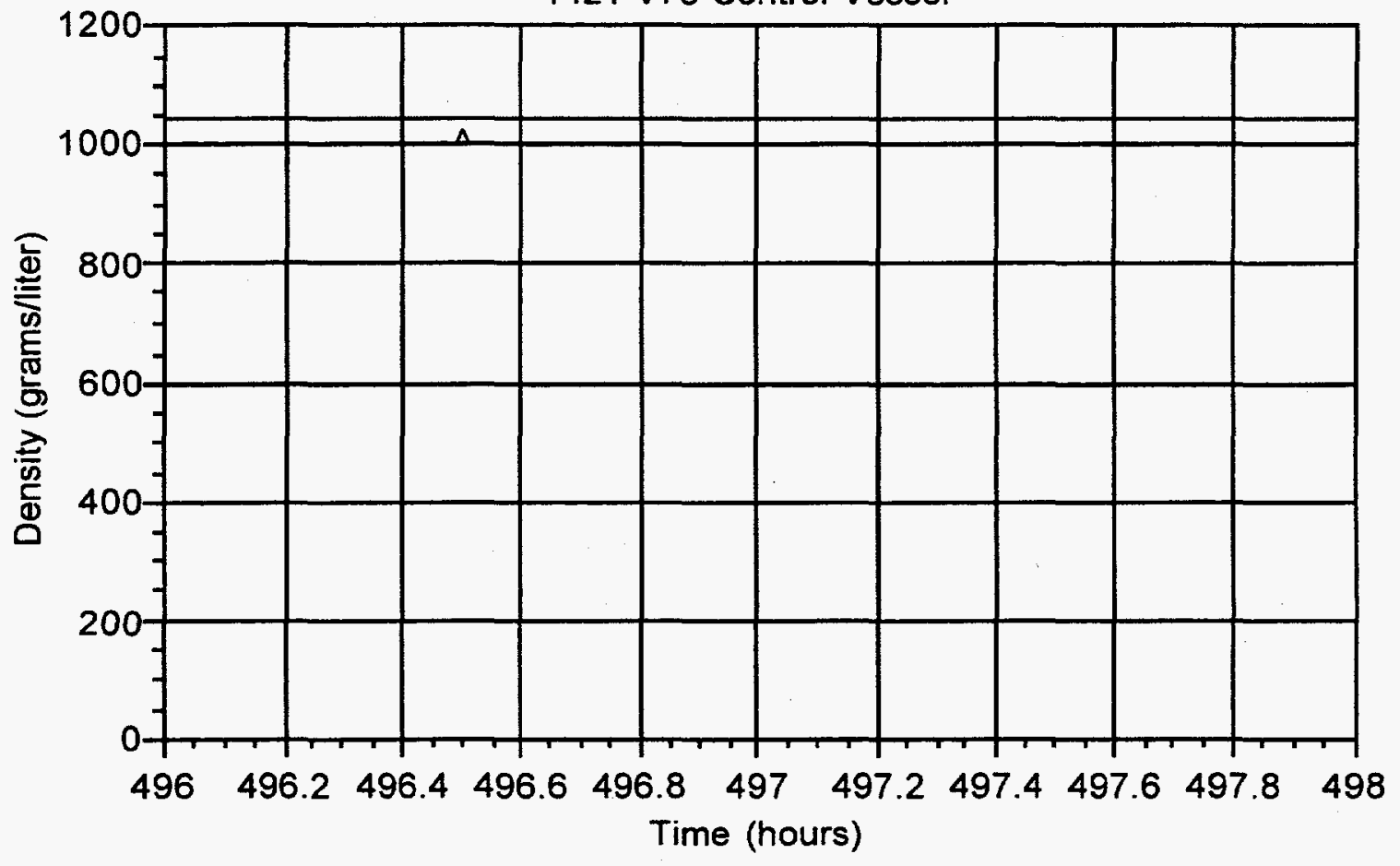

Fig. 3. Case 1e. 2.4-liter (0.3\%) diversion without replacement from control vessel 1421 V73 when the volume was nominally static. The diversion began at 496.4997 hours and lasted 5 minutes. 
1441 V70 Accountabiity Vessel

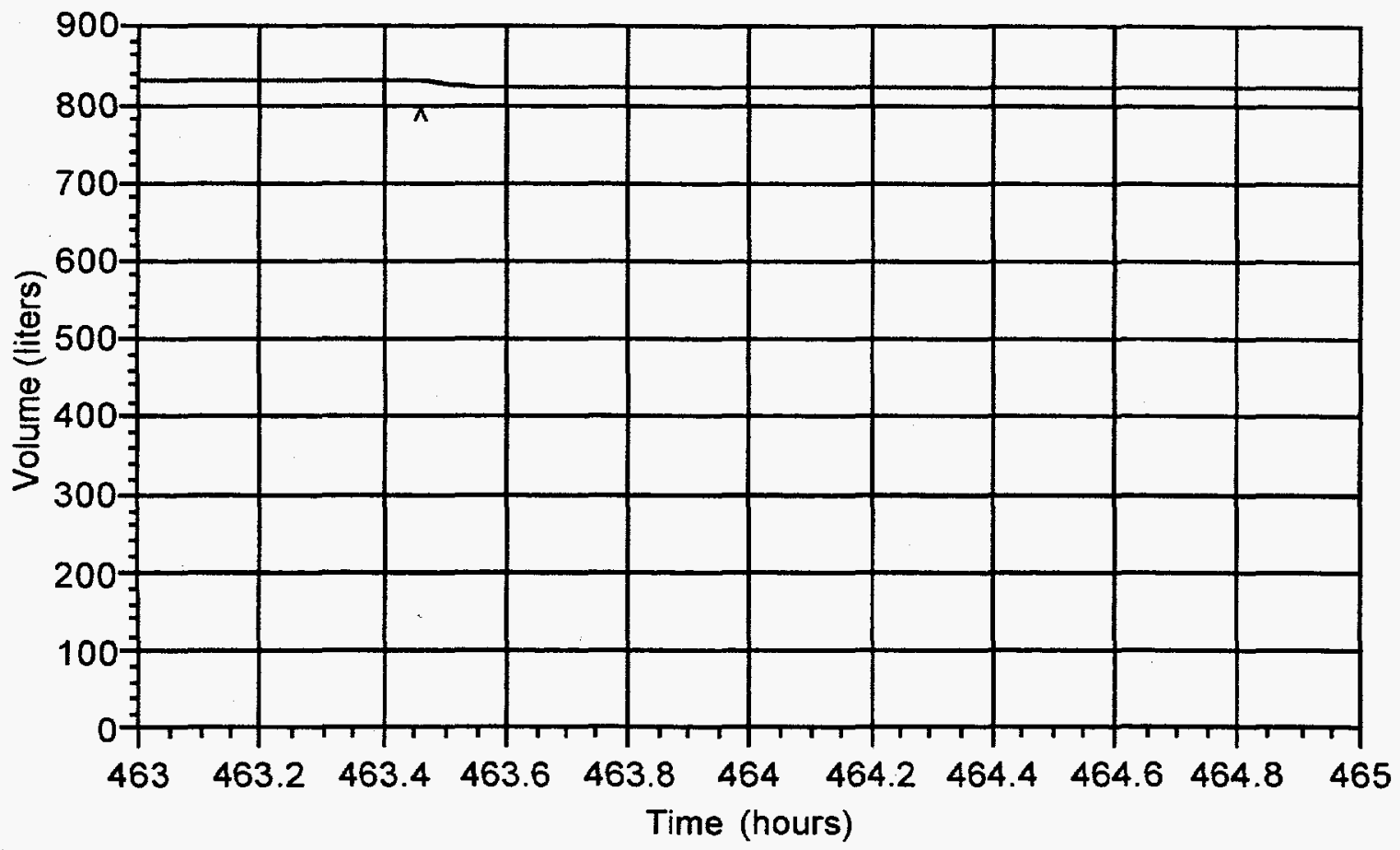

1441 V70 Accountability Vessel

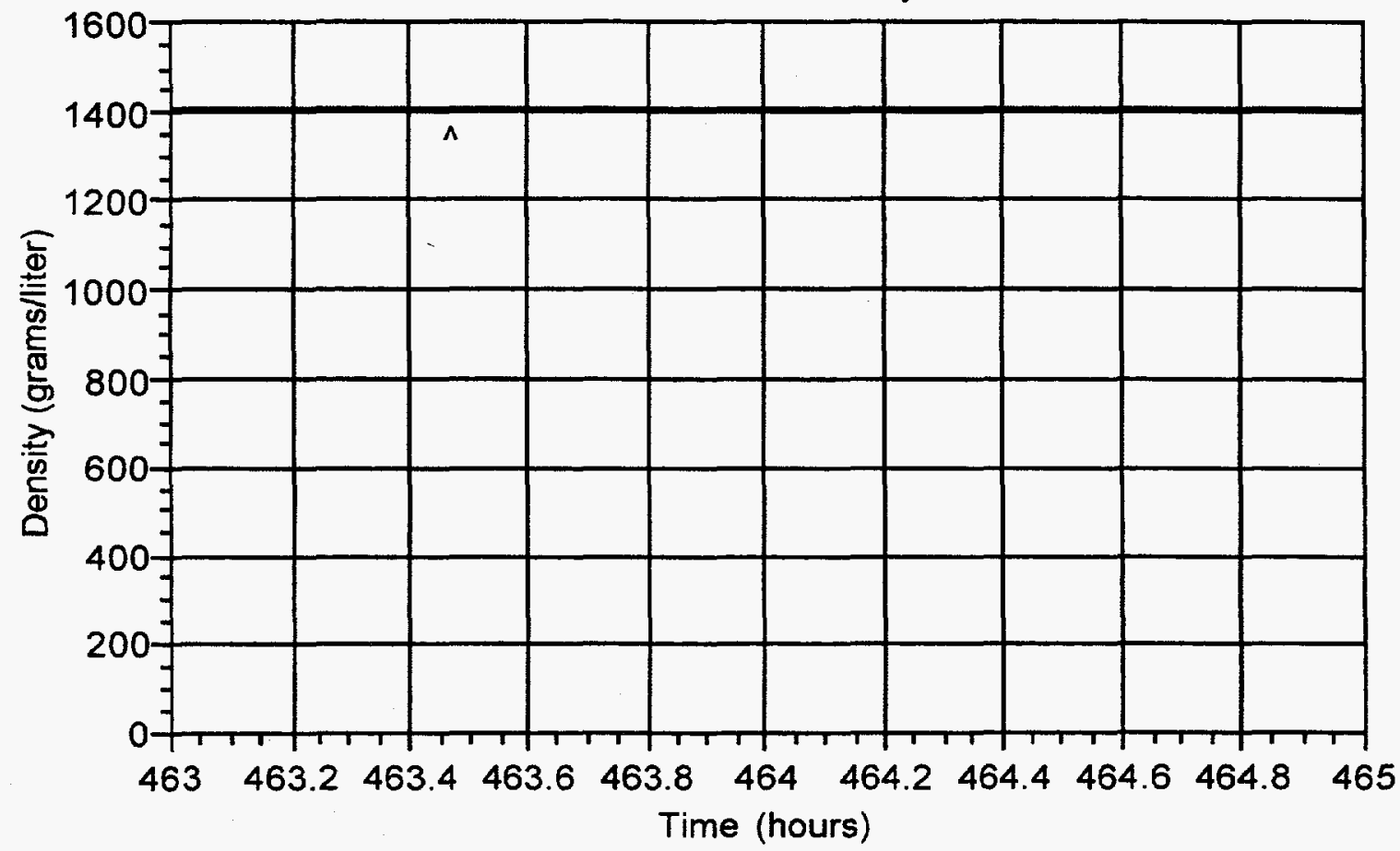

Fig. 4. Case 1f. 8.3-liter (1.0\%) diversion without replacement from the output accountability vessel 1441 V70 when the volume was nominally static. The diversion began at 463.4645 hours and lasted 5 minutes. 
1421 V73 Control Vessel

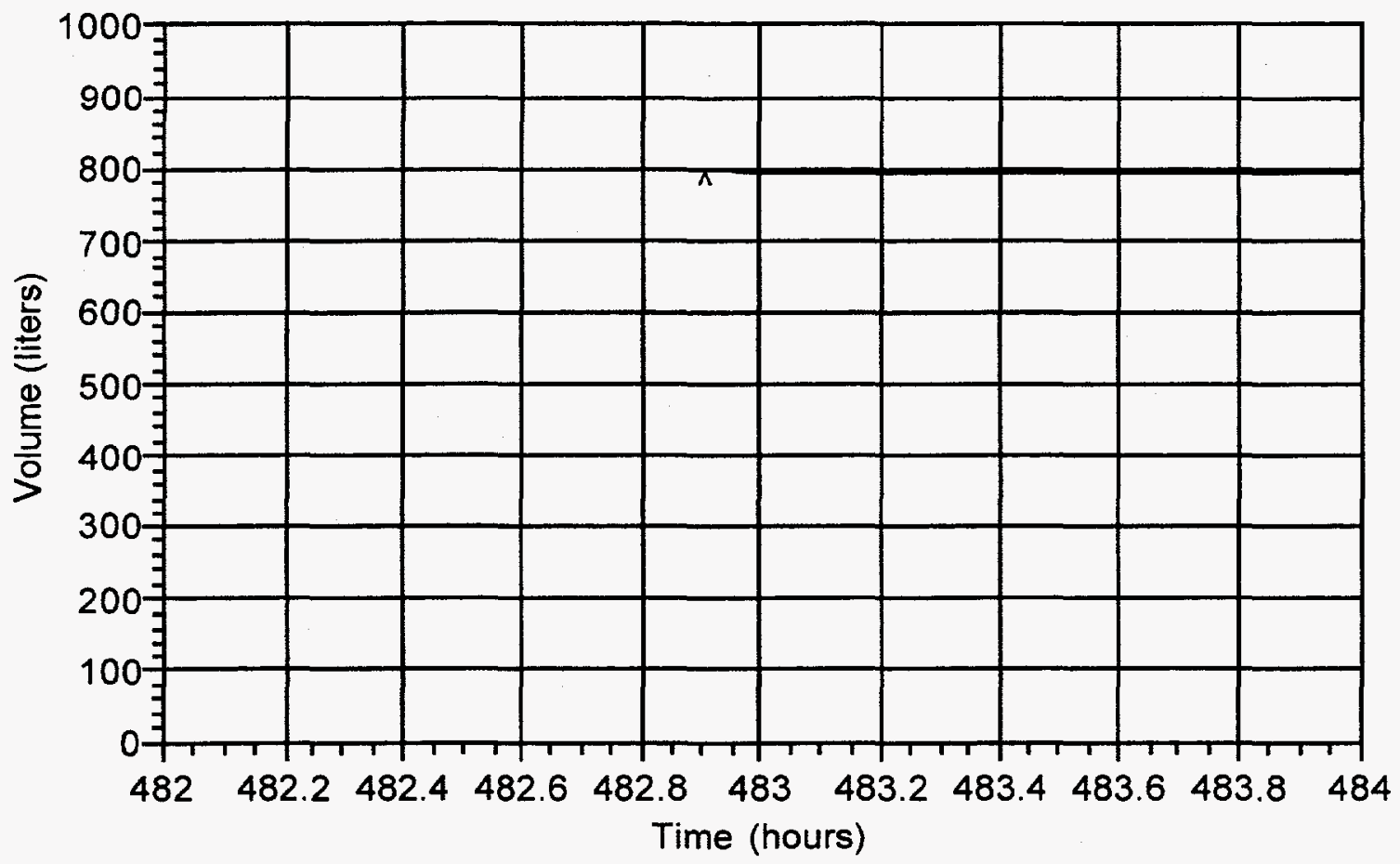

1421 V73 Control Vessel

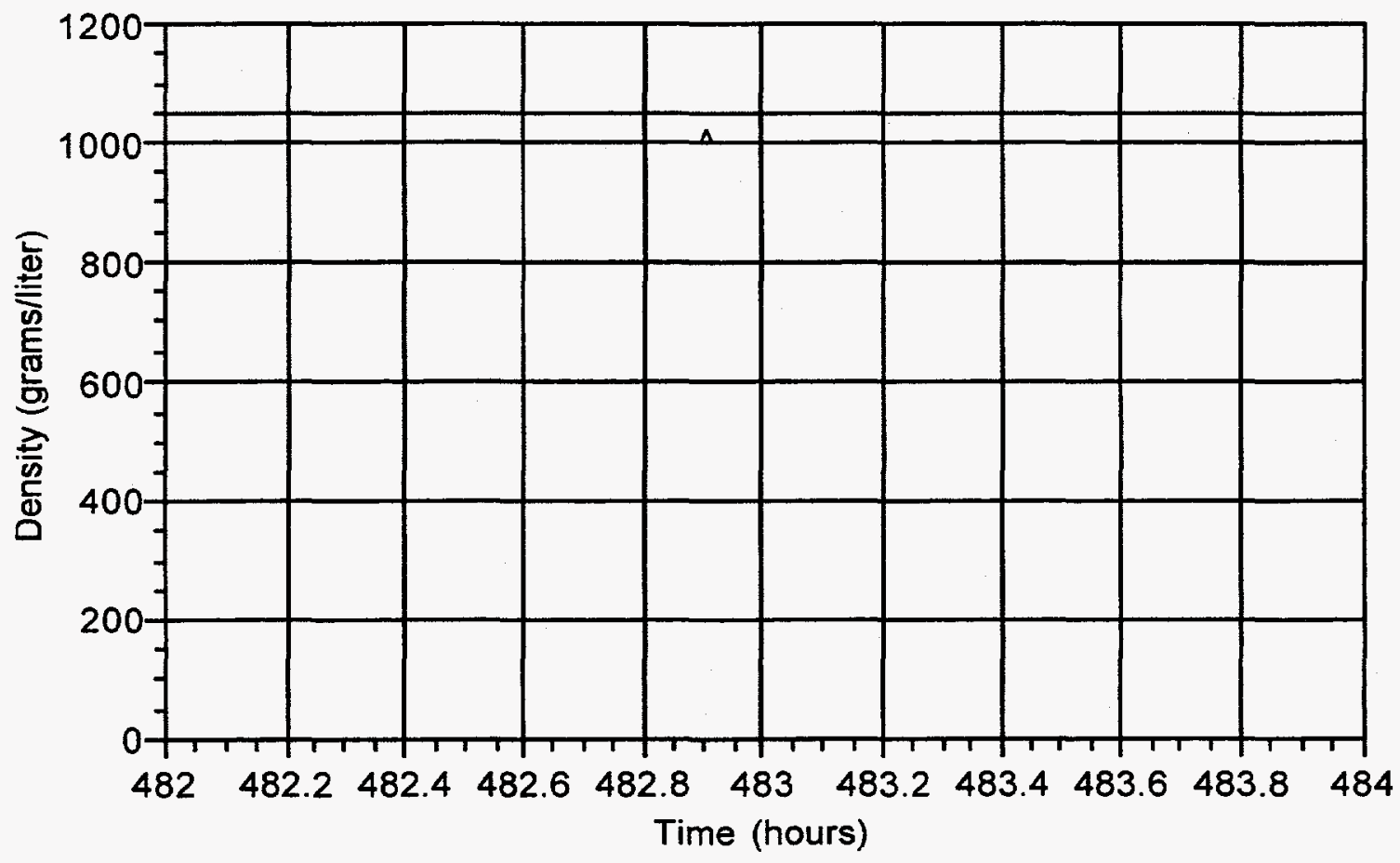

Fig. 5. Case 1h. 8.0-liter (1.0\%) diversion without replacement from control vessel 1421 V73 when the volume was nominally static. The diversion began at 482.9037 hours and lasted 5 minutes. 


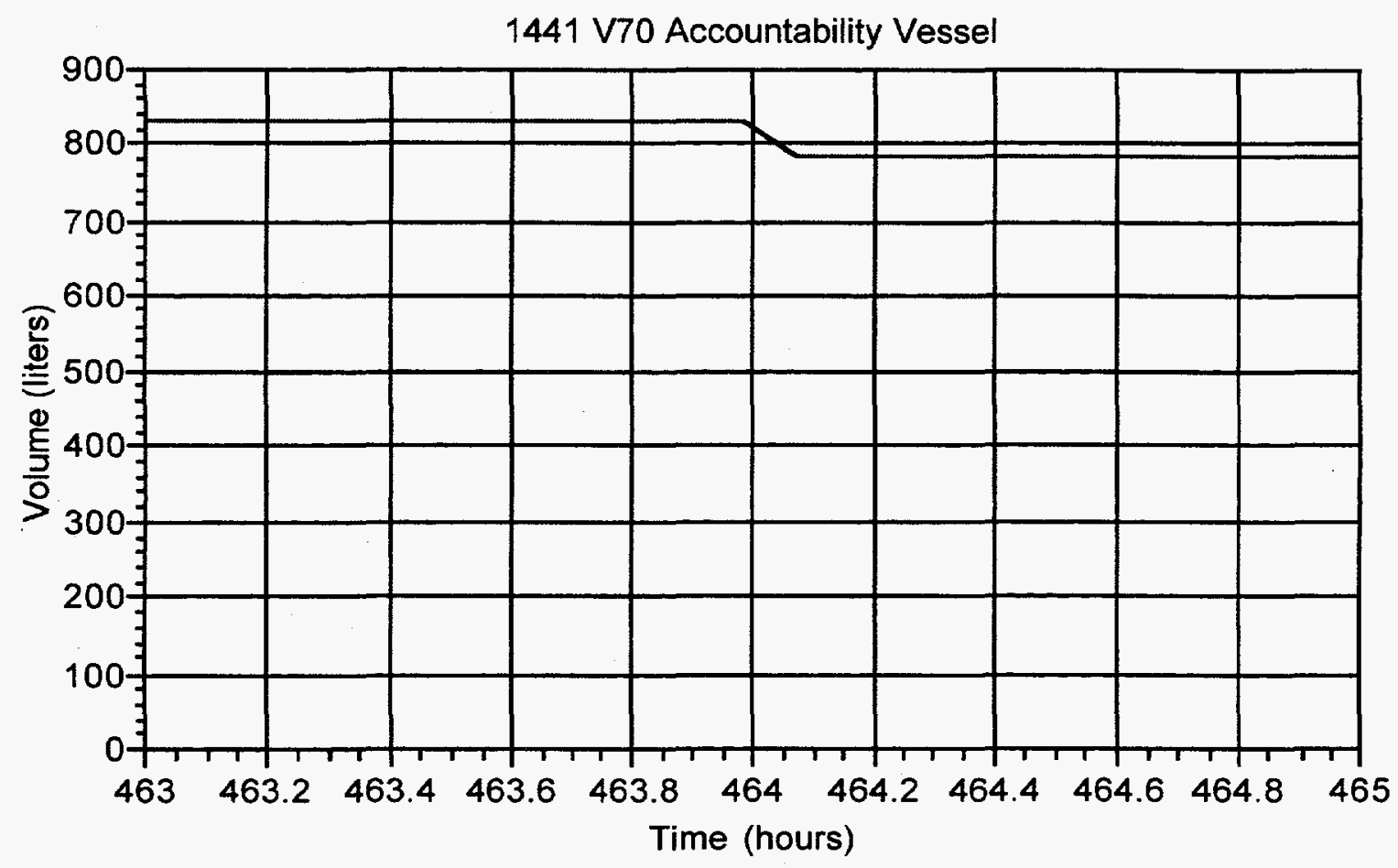

Fig. 6. Case 1i. 41.5-liter (5.0\%) diversion without replacement from the output accountability vessel 1441 V70 when the volume was nominally static. The diversion began at 463.9861 hours and lasted 5 minutes. 
1211 V55 Control Vessel

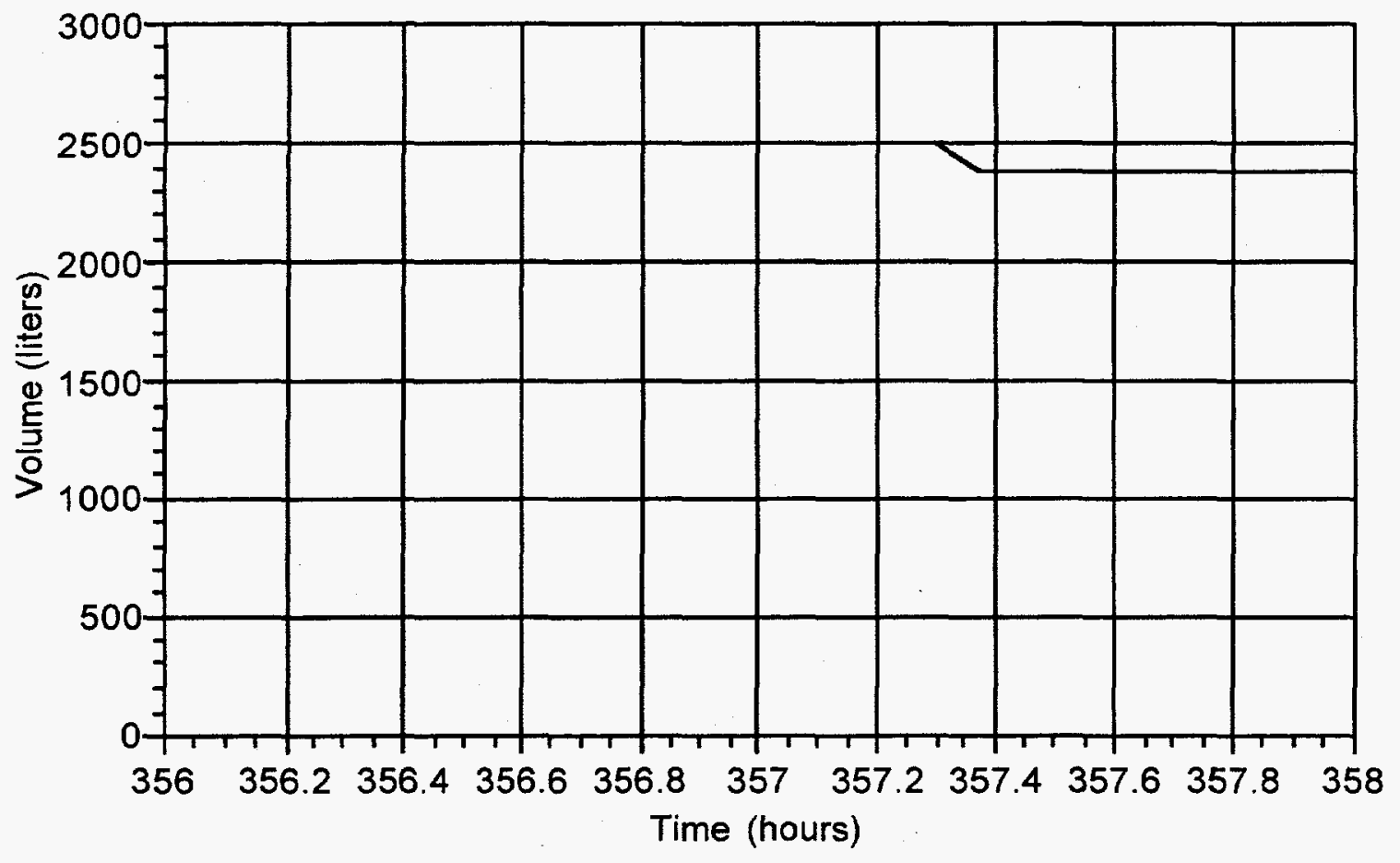

1211 V55 Control Vessel

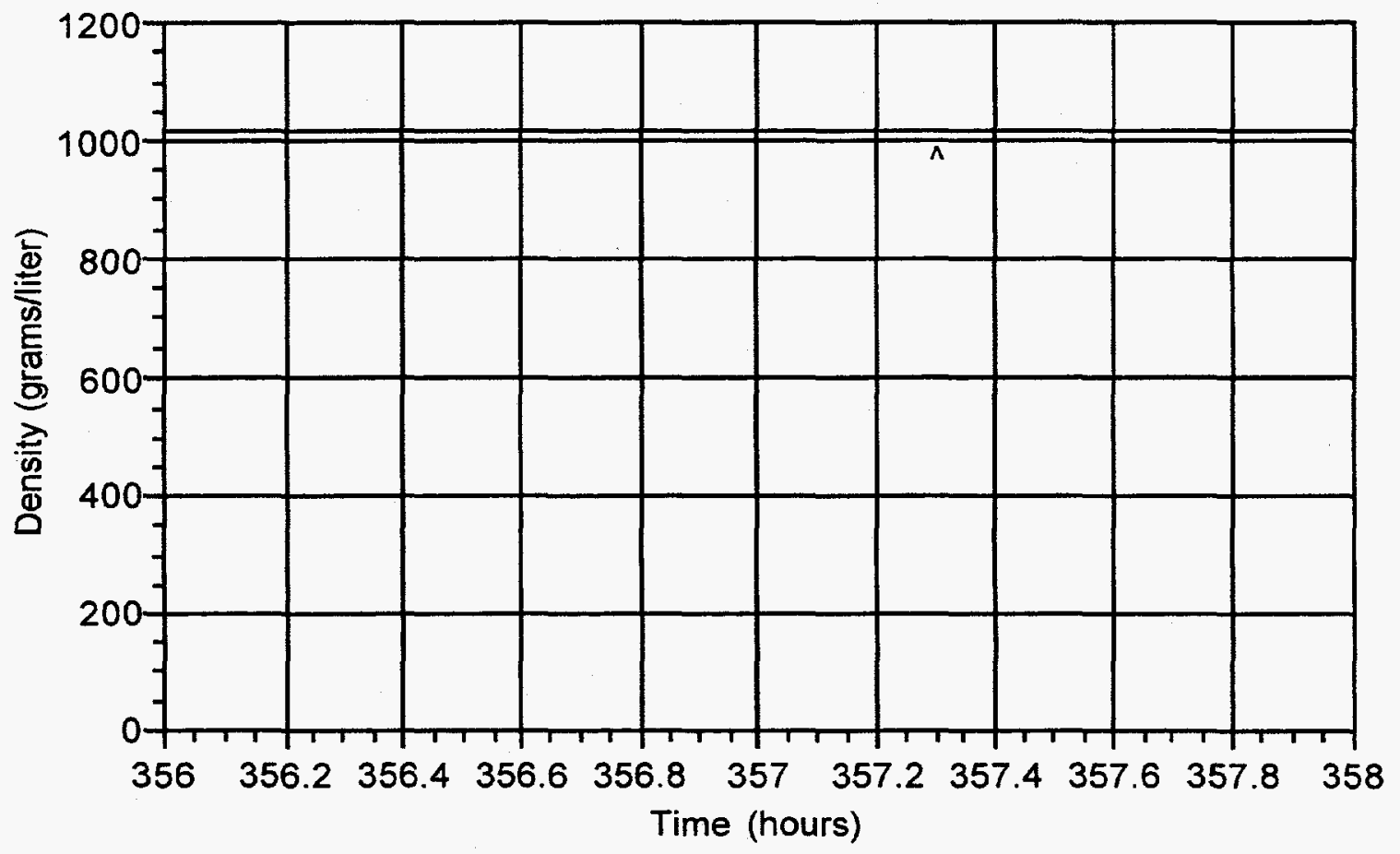

Fig. 7. Case 1k. 125-liter (5.0\%) diversion without replacement from control vessel 1211 V55 when the volume was nominally static. The diversion began at 357.3125 hours and lasted 5 minutes. 
1441 V70 Accountability Vessel

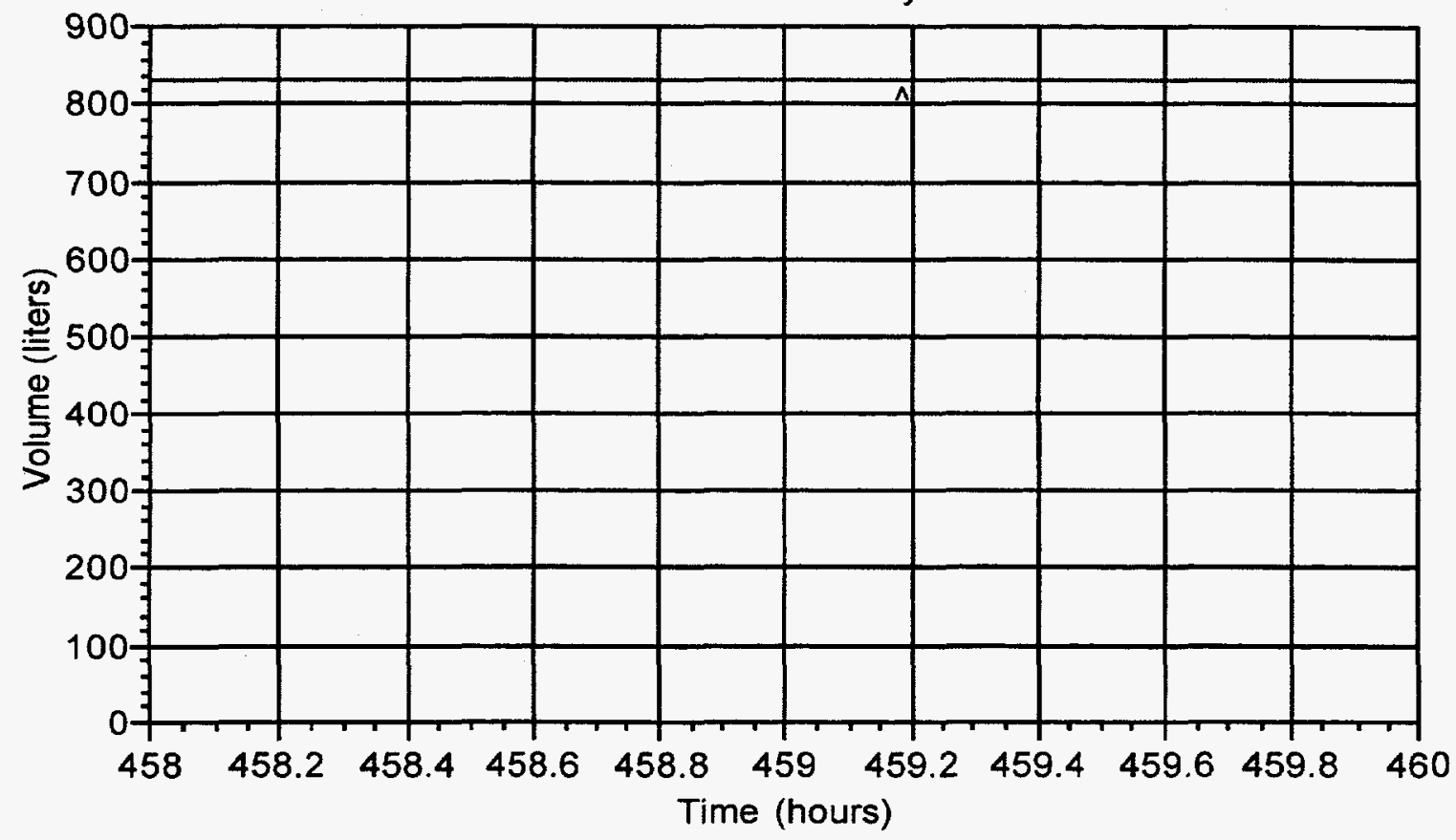

1441 V70 Accountability Vessel

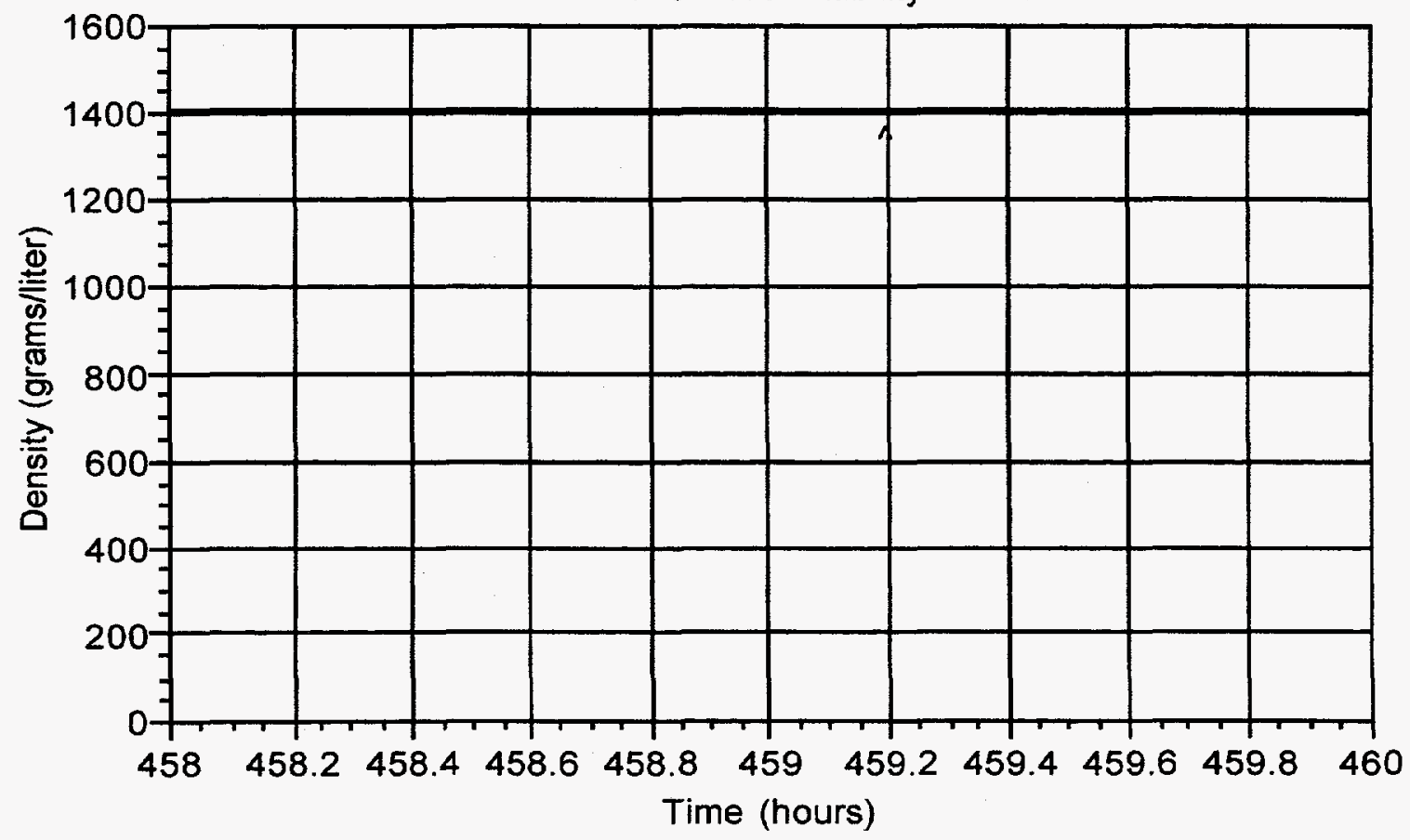

Fig. 8. Case. 2a. 0.83-liter ( $0.1 \%)$ diversion with replacement from the output accountability vessel 1441 V70 when the volume was nominally static. The diversion began at 459.1938 hours and lasted 5 minutes. The diverted solution was replaced with an equal volume of 3-molar nitric acid, which caused a density decrease on the order of $0.01 \%$. 
1441 V70 Accountability Vessel

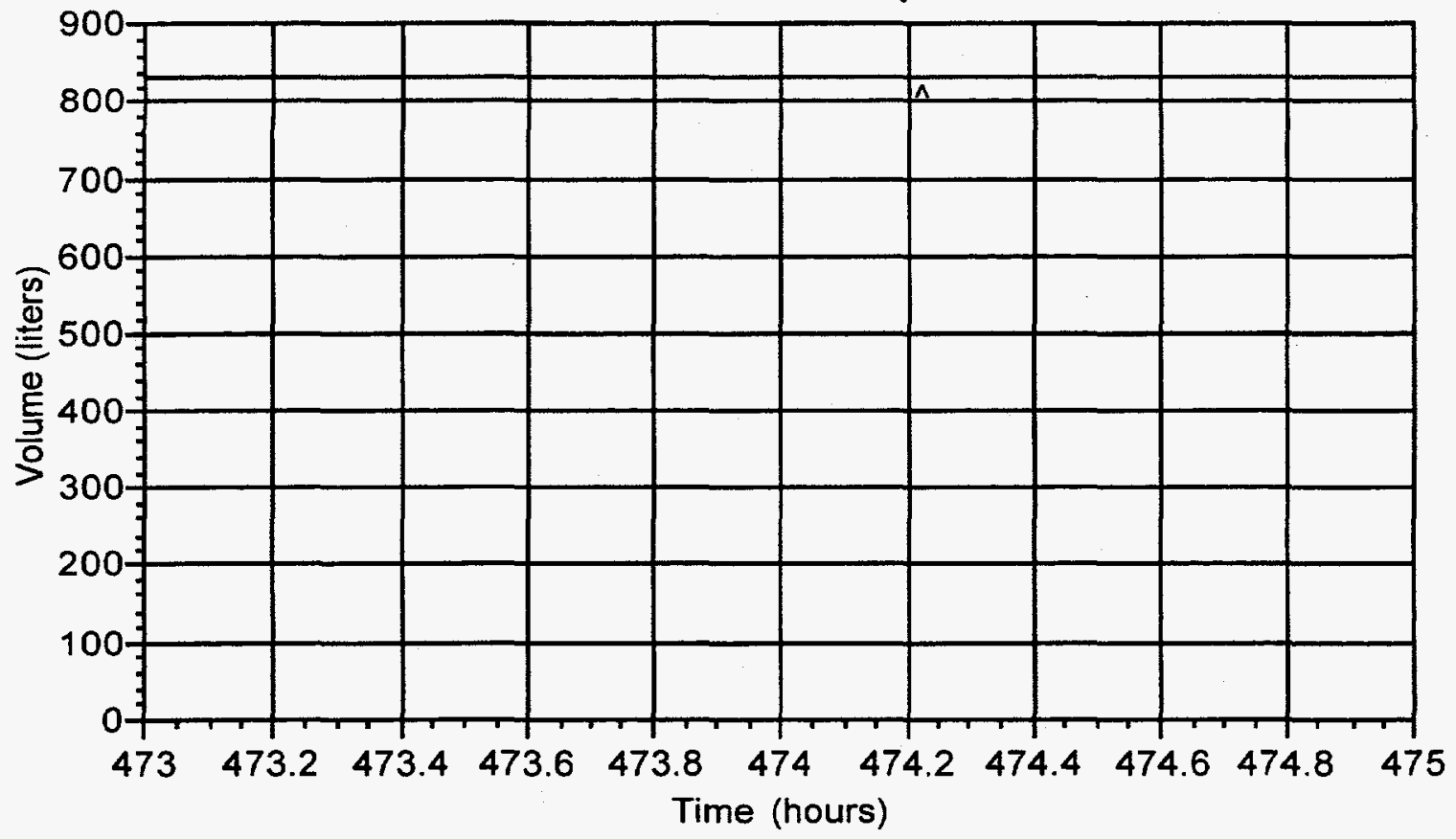

1441 V70 Accountability Vessel

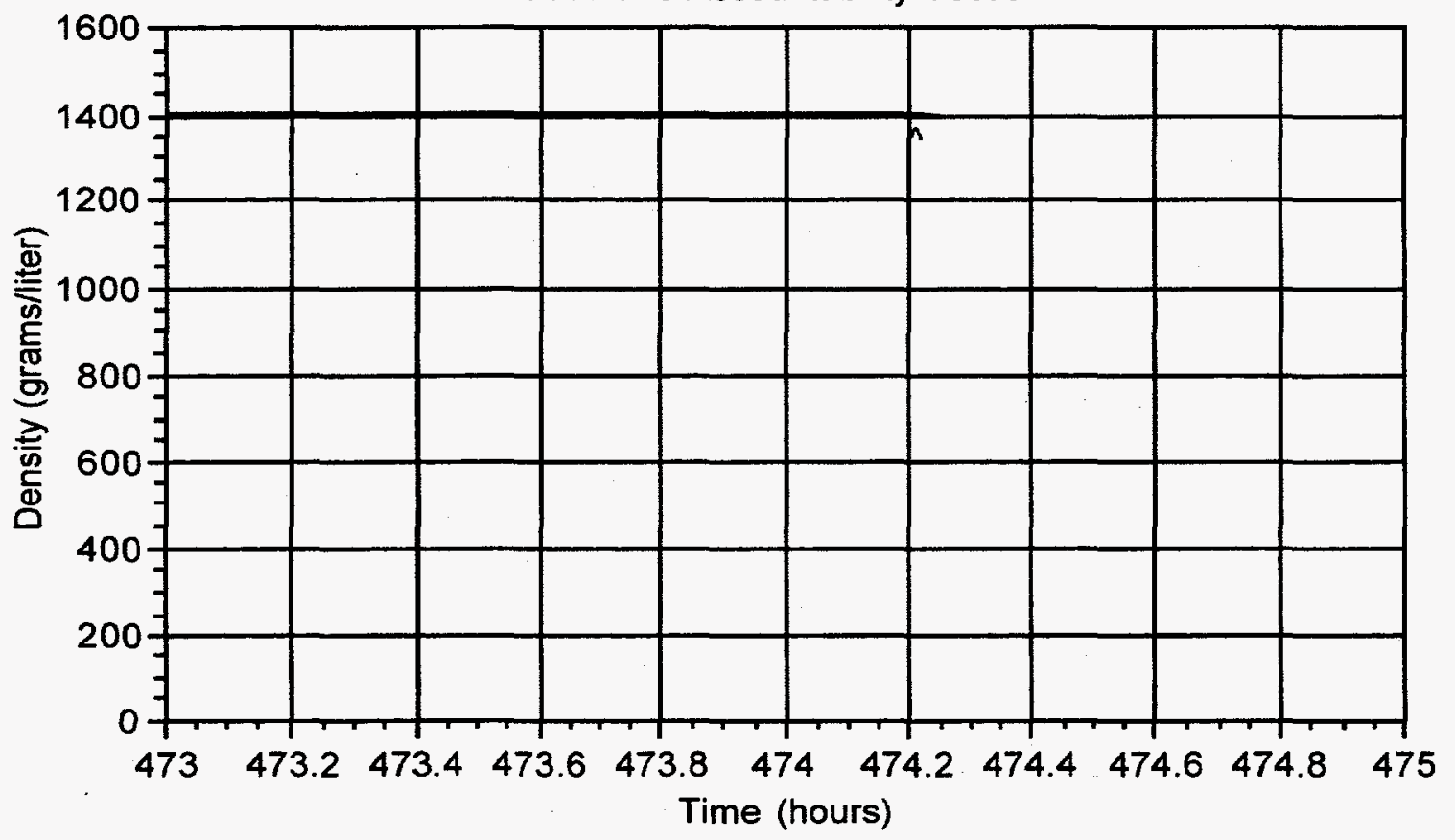

Fig. 9. Case 2c. 2.49-liter (0.3\%) diversion with replacement from the output accountability vessel 1441 V70 when the volume was nominally static. The diversion began at 474.2074 hours and lasted 5 minutes. The diverted solution was replaced with an equal volume of 3-molar nitric acid, which caused a density decrease on the order of $0.05 \%$. 
1421 V73 Control Vessel

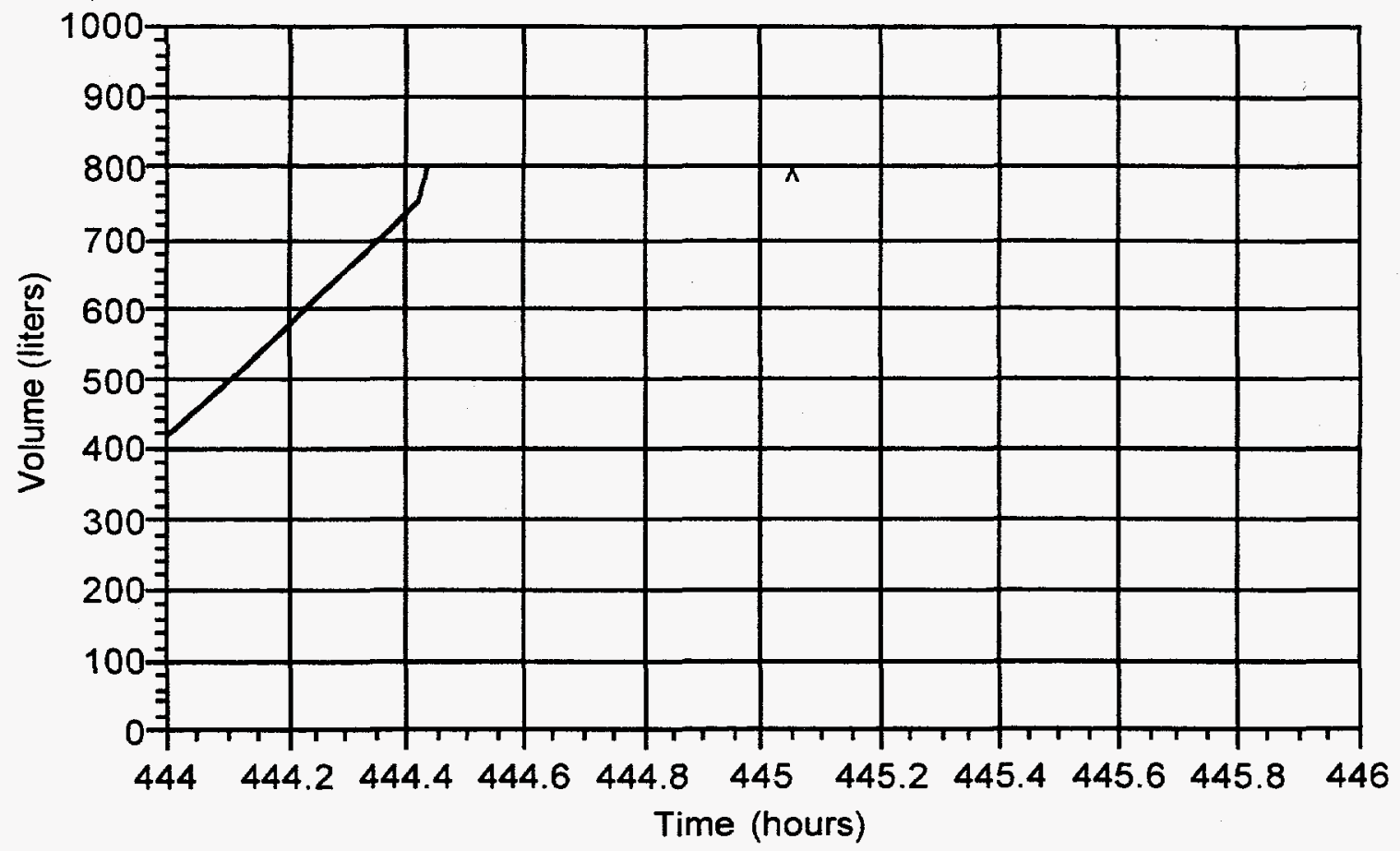

1421 V73 Control Vessel

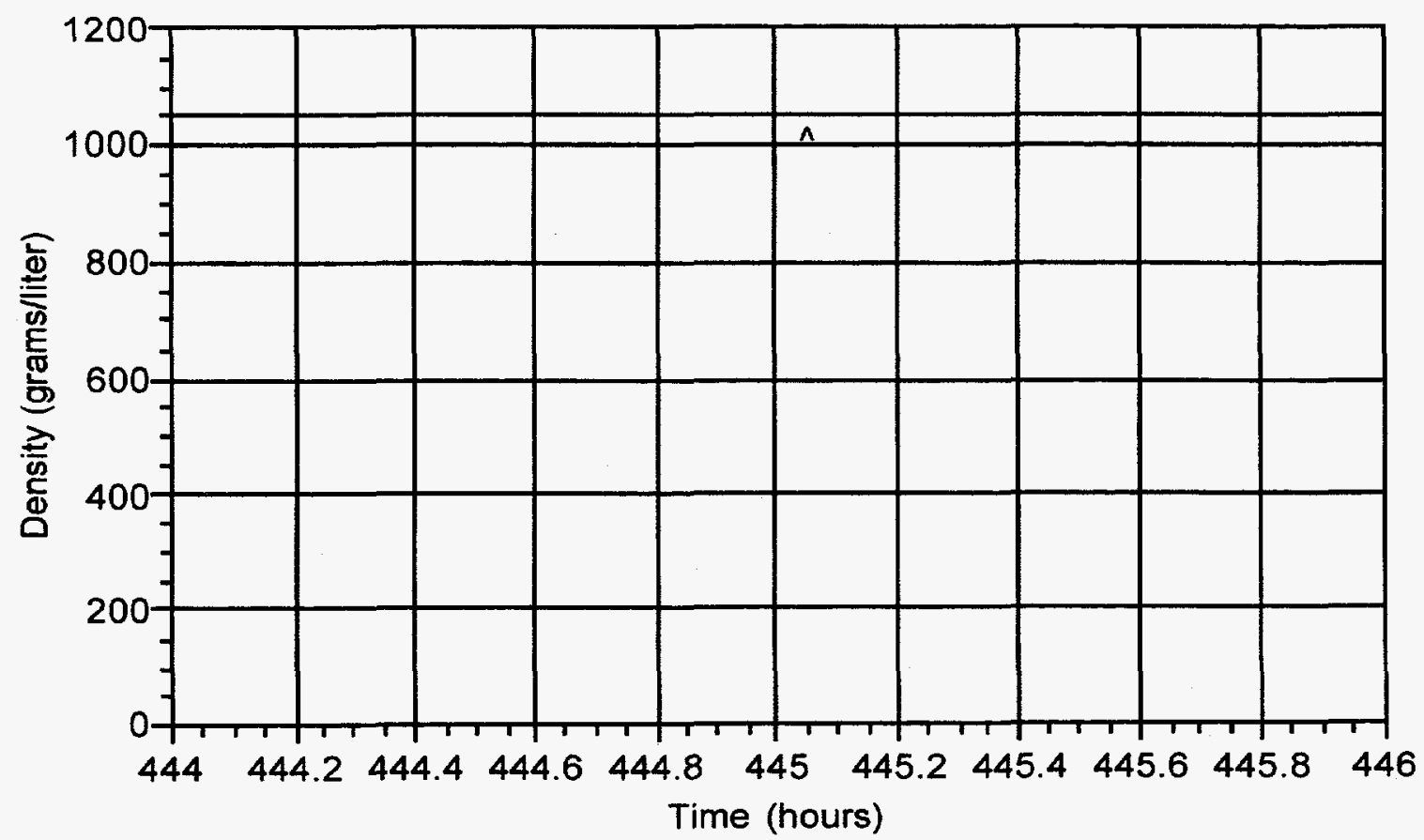

Fig. 10. Case 2d. 2.4-liter (0.3\%) diversion with replacement from control vessel 1421 V73 when the volume was nominally static. The diversion began at 445.0194 hours and lasted 5 minutes. The diverted solution was replaced with an equal volume of 3-molar nitric acid, which caused a density decrease of less than $0.01 \%$. 
1441 V70 Accountability Vessel

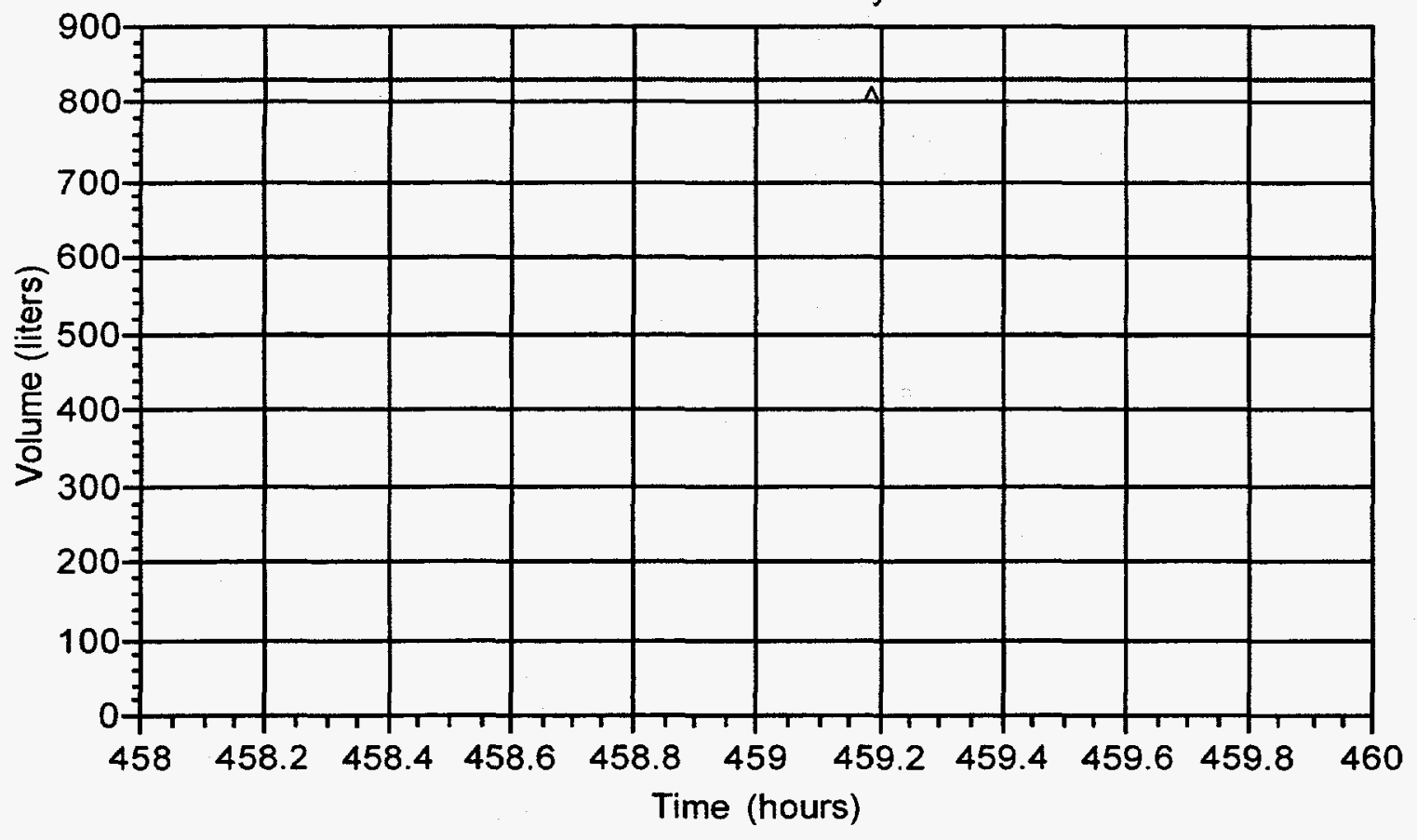

1441 V70 Accountability Vessel

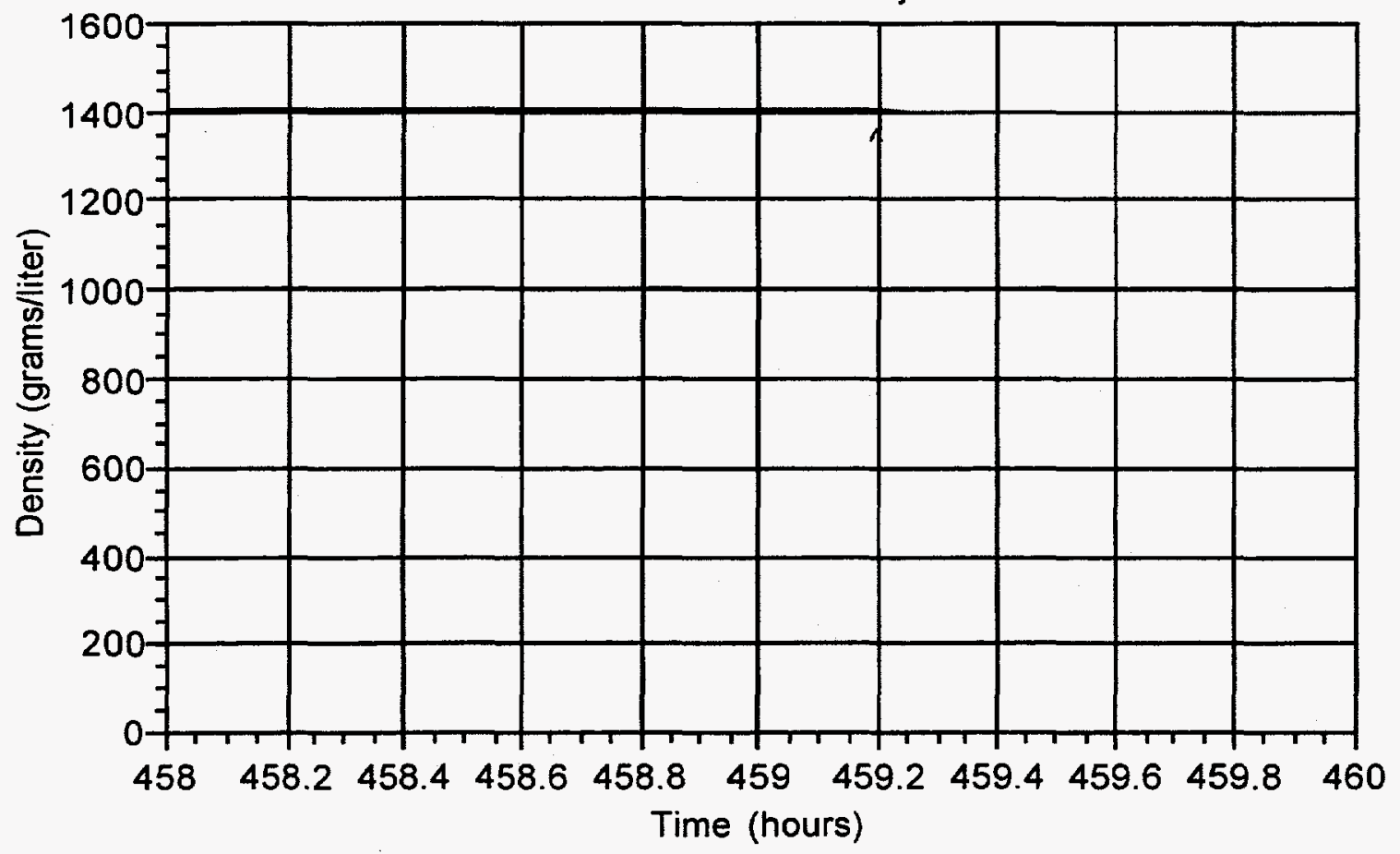

Fig. 11, Case 2e. 8.3-liter (1.0\%) diversion with replacement from output accountability vessel 1441 V70

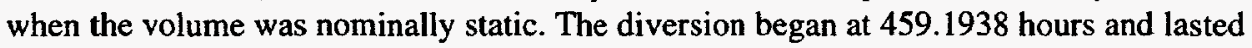
5 minutes. The diverted solution was replaced with an equal volume of 3 -molar nitric acid, which caused a density decrease of $0.19 \%$. 


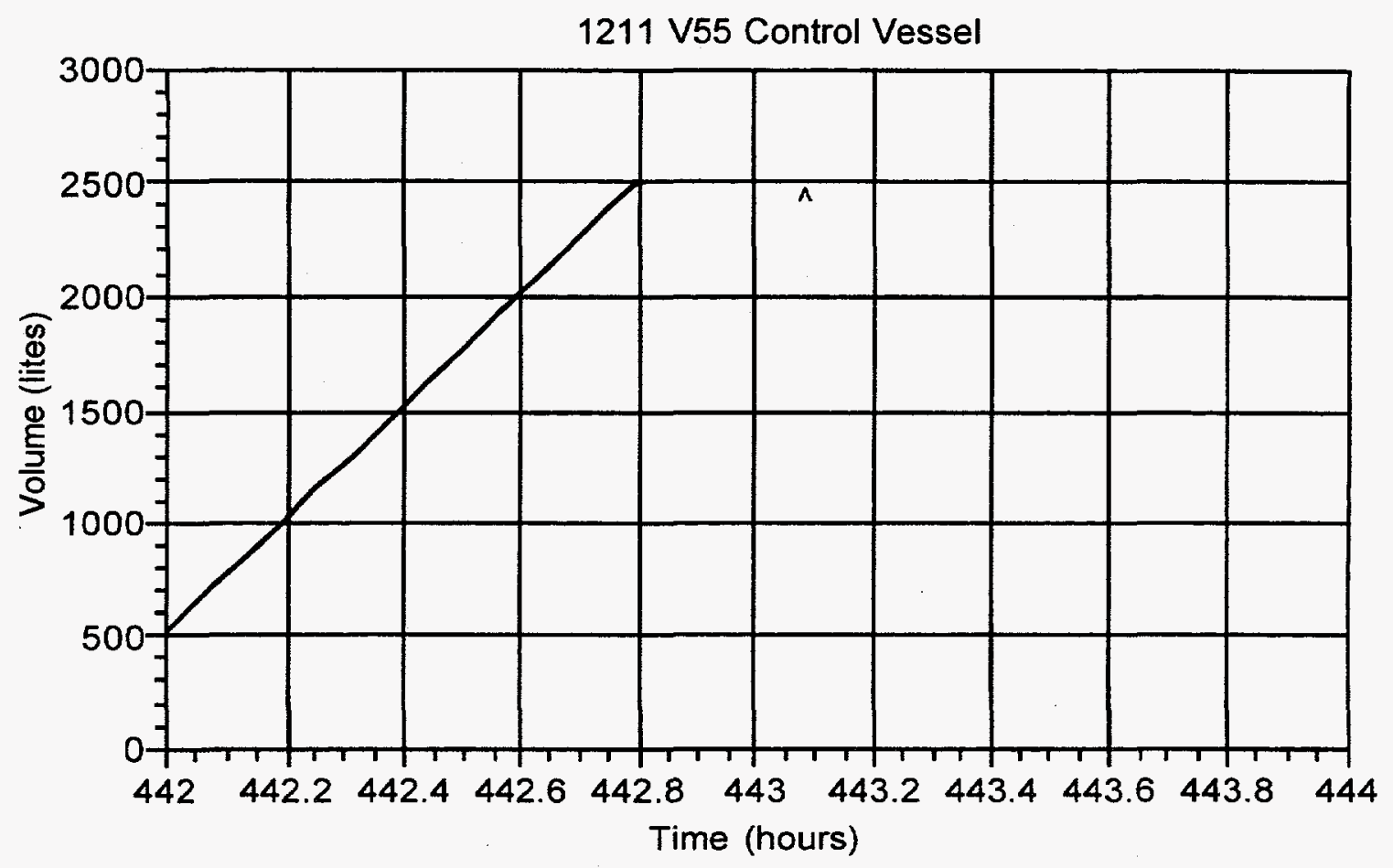

1211 V55 Control Vessel

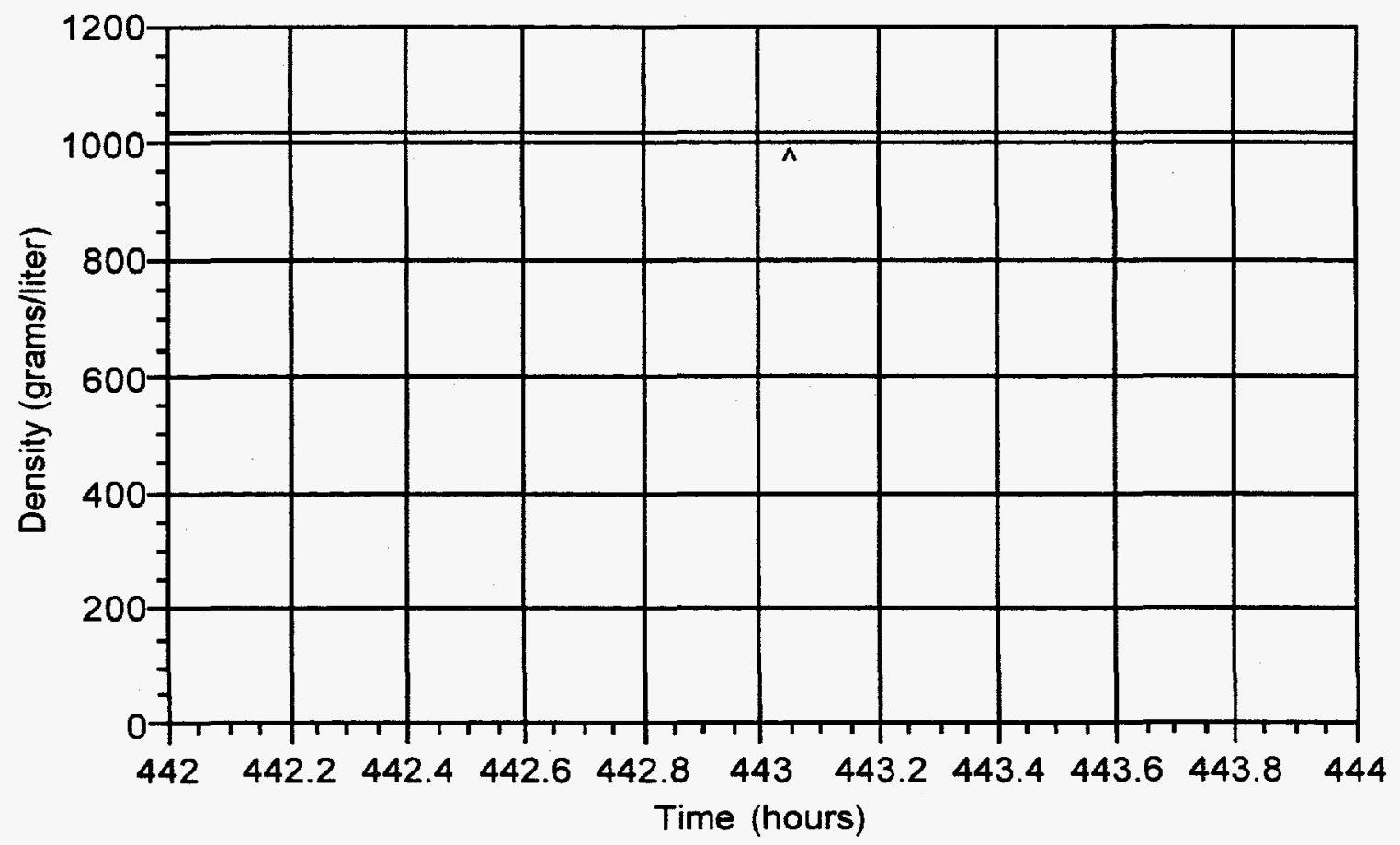

Fig. 12. Case 2f. 25-liter (1.0\%) diversion with replacement from control vessel 1211 V55 when the volume was nominally static. The diversion began at 443.0313 hours and lasted 5 minutes. The diverted solution was replaced with an equal volume of 3-molar nitric acid, which caused a density increase of $0.01 \%$. 
1441 V70 Accountabilitiy Vessel

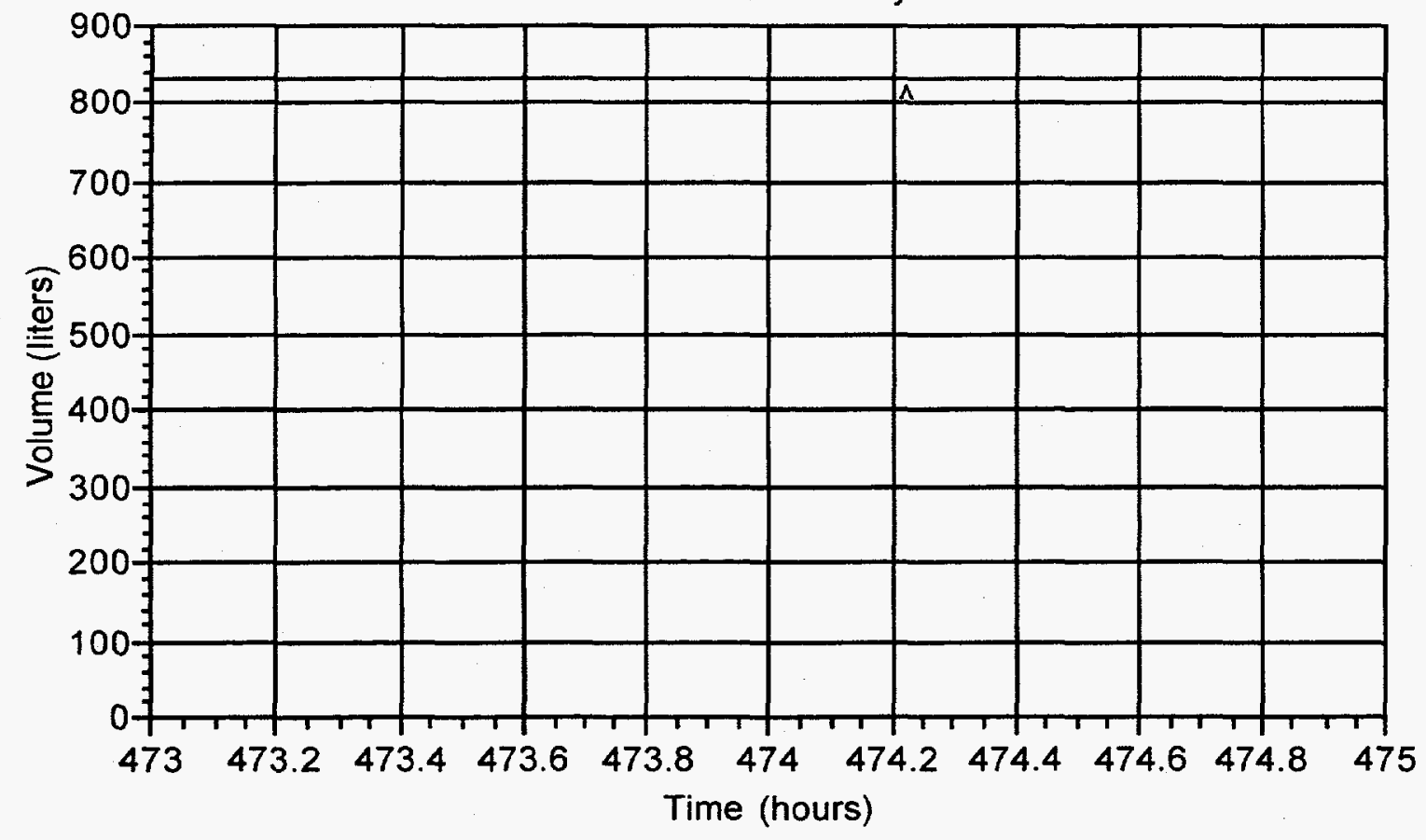

1441 V70 Accountability Vessel

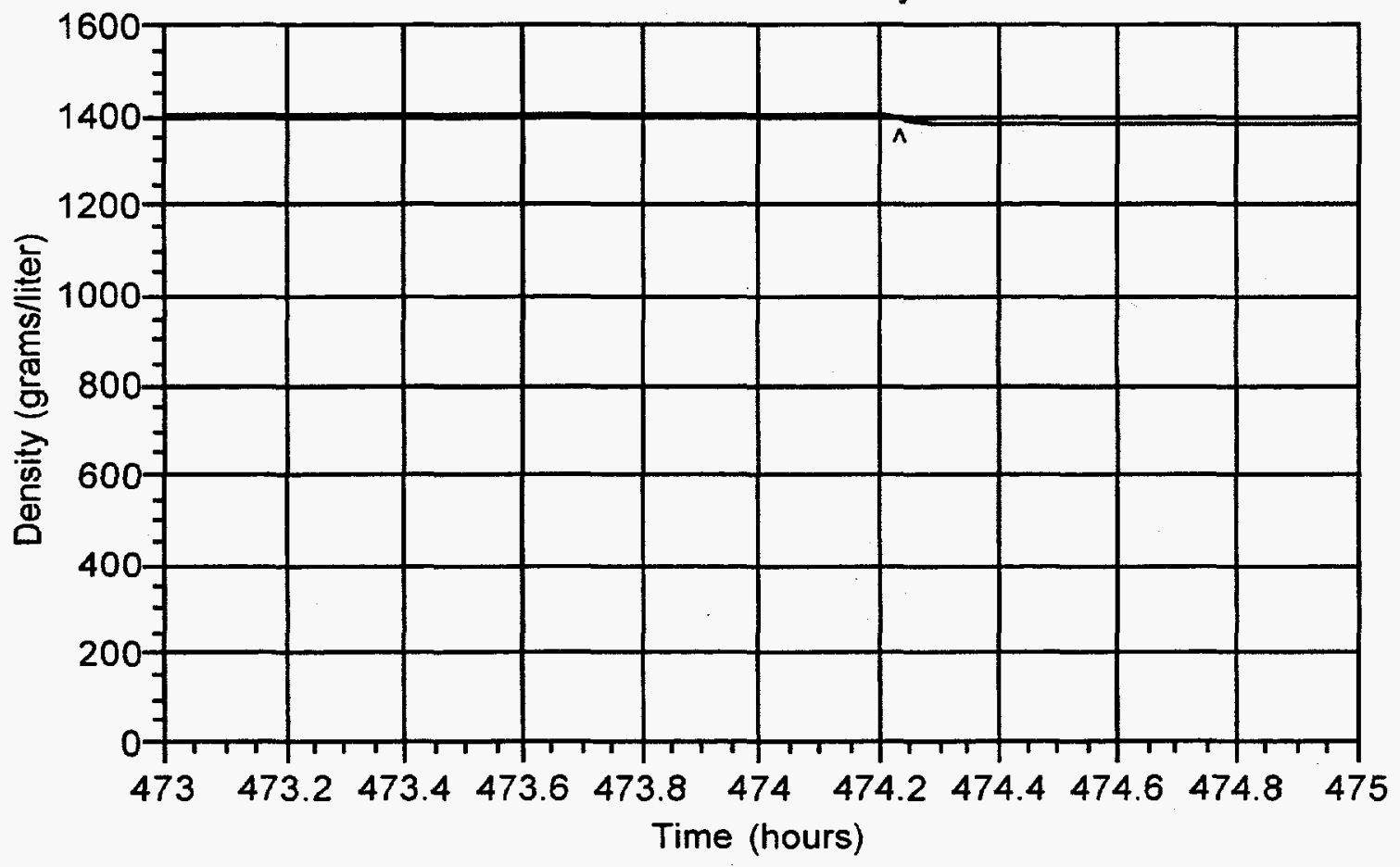

Fig. 13. Case 2g. 41.5-liter (5.0\%) diversion with replacement from output accountability vessel 1441 V70 when the volume was nominally static. The diversion began at 474.2074 hours and lasted 5 minutes. The diverted solution was replaced with an equal volume of 3-molar nitric acid, which caused a density decrease of $0.96 \%$. 
1421 V71 Control Vessel

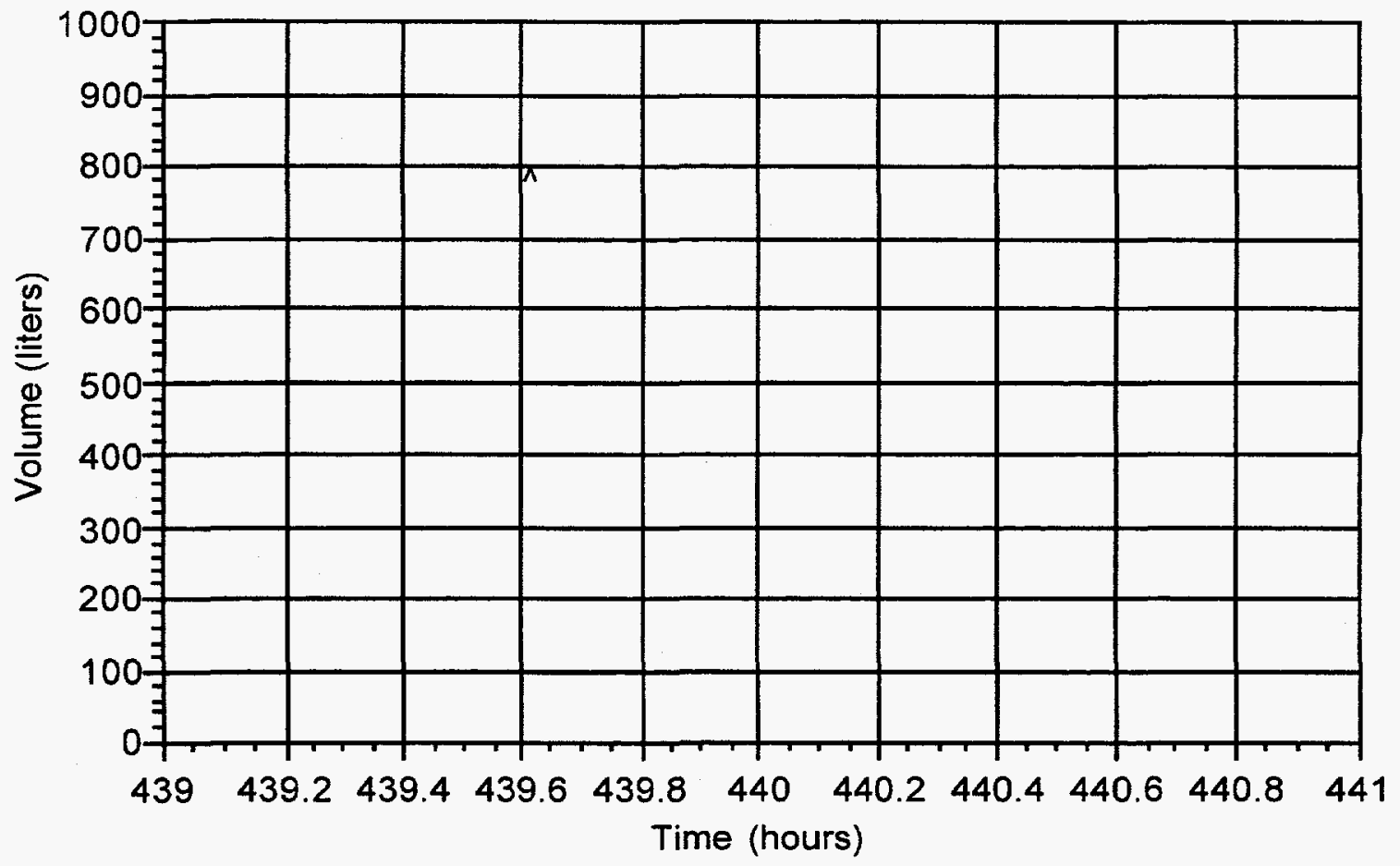

1421 V71 Control Vessel

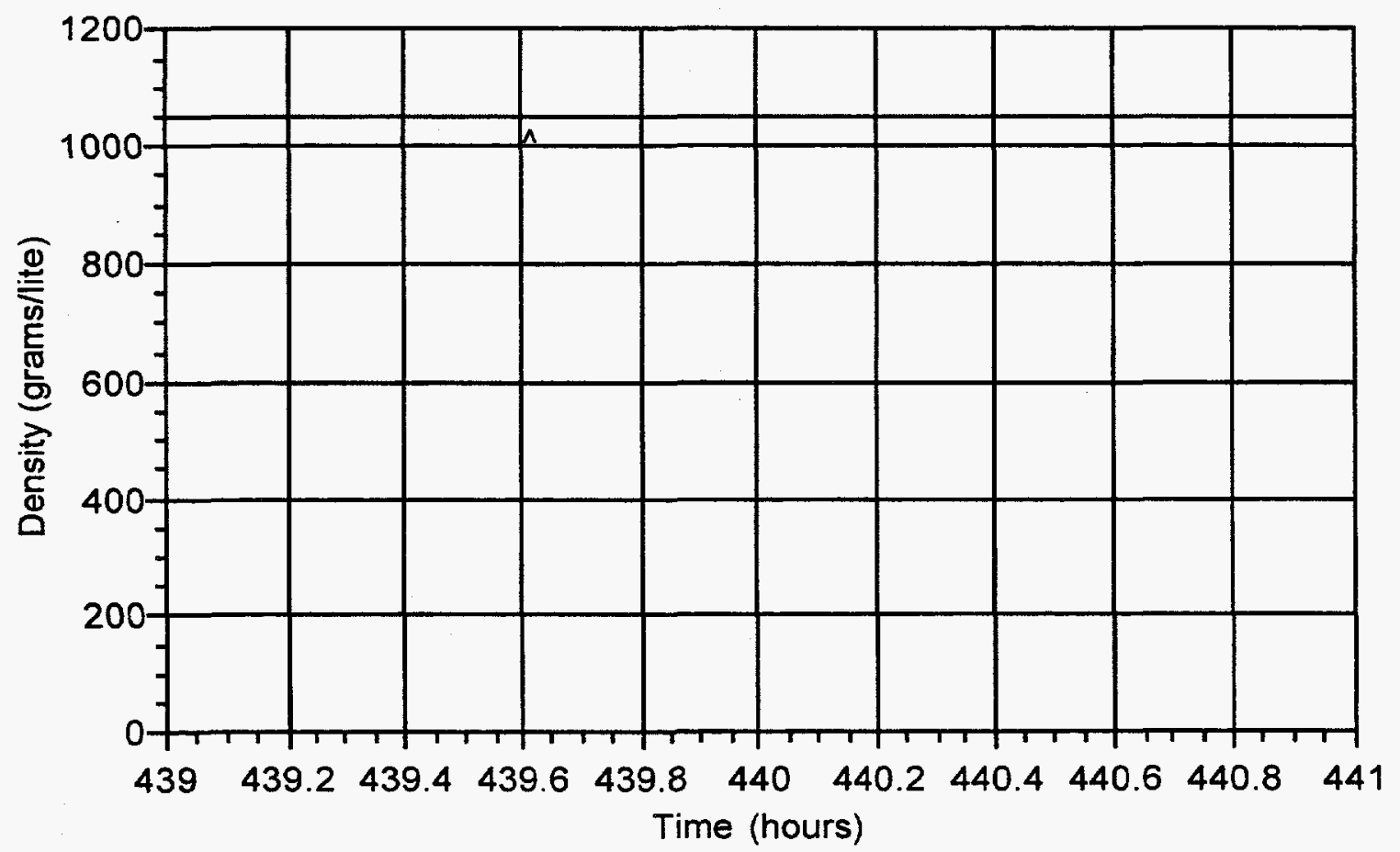

Fig. 14. Case 2h. 40-liter (1.0\%) diversion with replacement from buffer vessel 1421 V71 when the volume was nominally static. The diversion began at 439.6040 hours and lasted 5 minutes. The diverted solution was replaced with an equal volume of 3-molar nitric acid, which caused a density increase of $0.29 \%$. 
1441 V70 Accountability Vessel

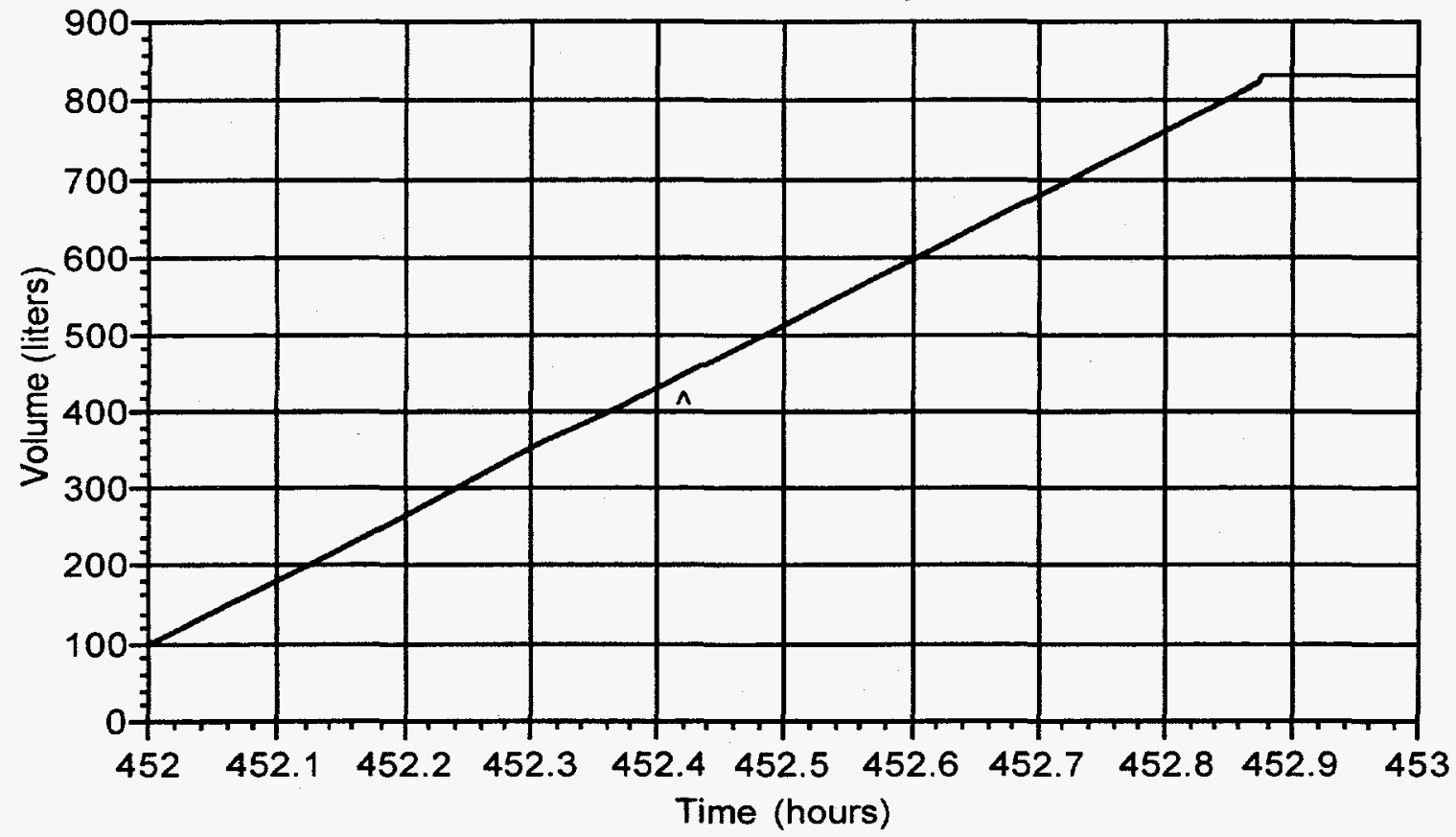

1441 V70 Accountability Vessel

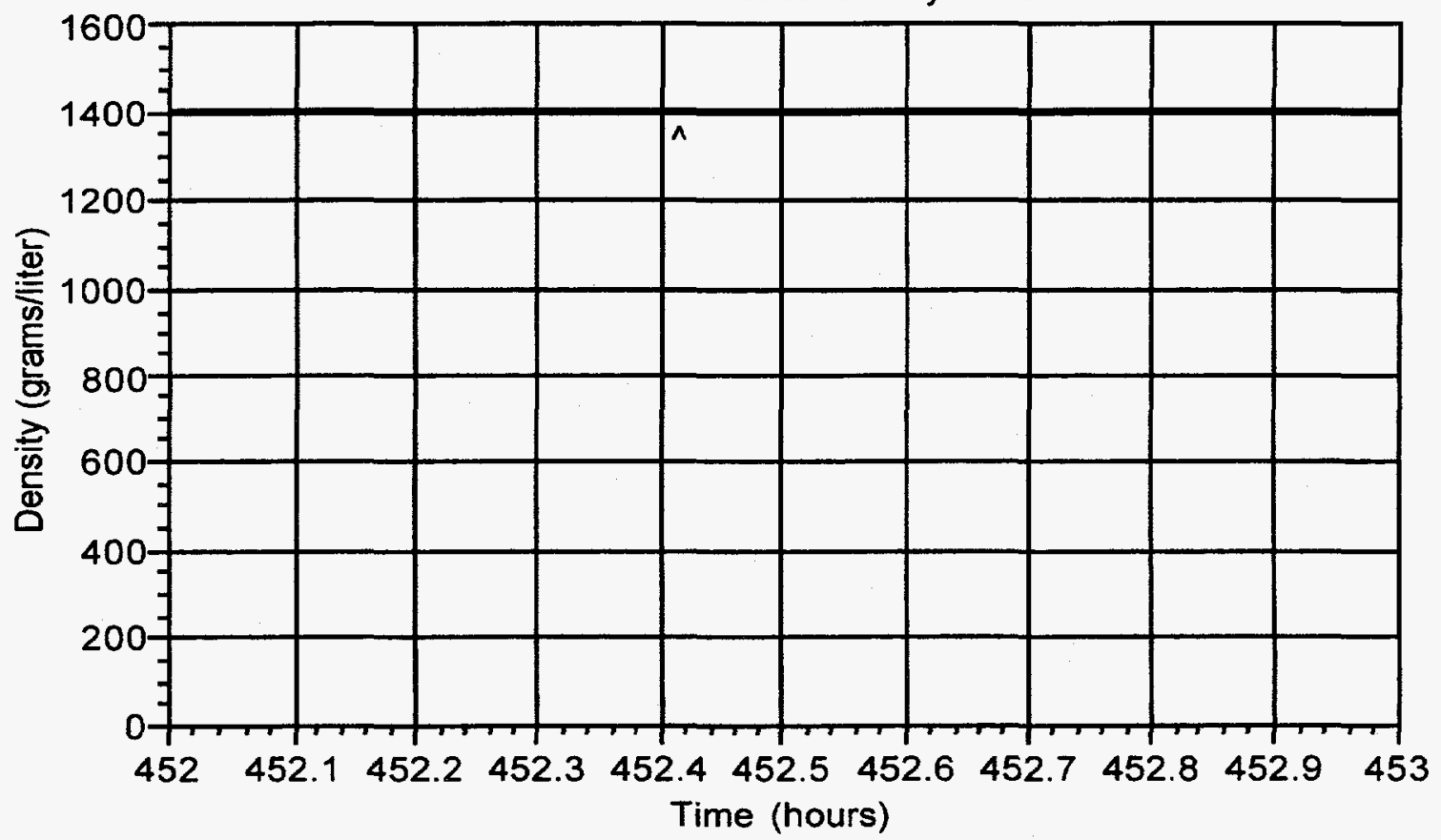

Fig. 15. Case 3a-1. 0.83-liter (0.1\%) diversion without replacement from output accountability vessel 1441 V70 during an input transfer. The diversion began at 452.4351 hours and lasted 5 minutes. 
1441 V70 Accountability Vessel

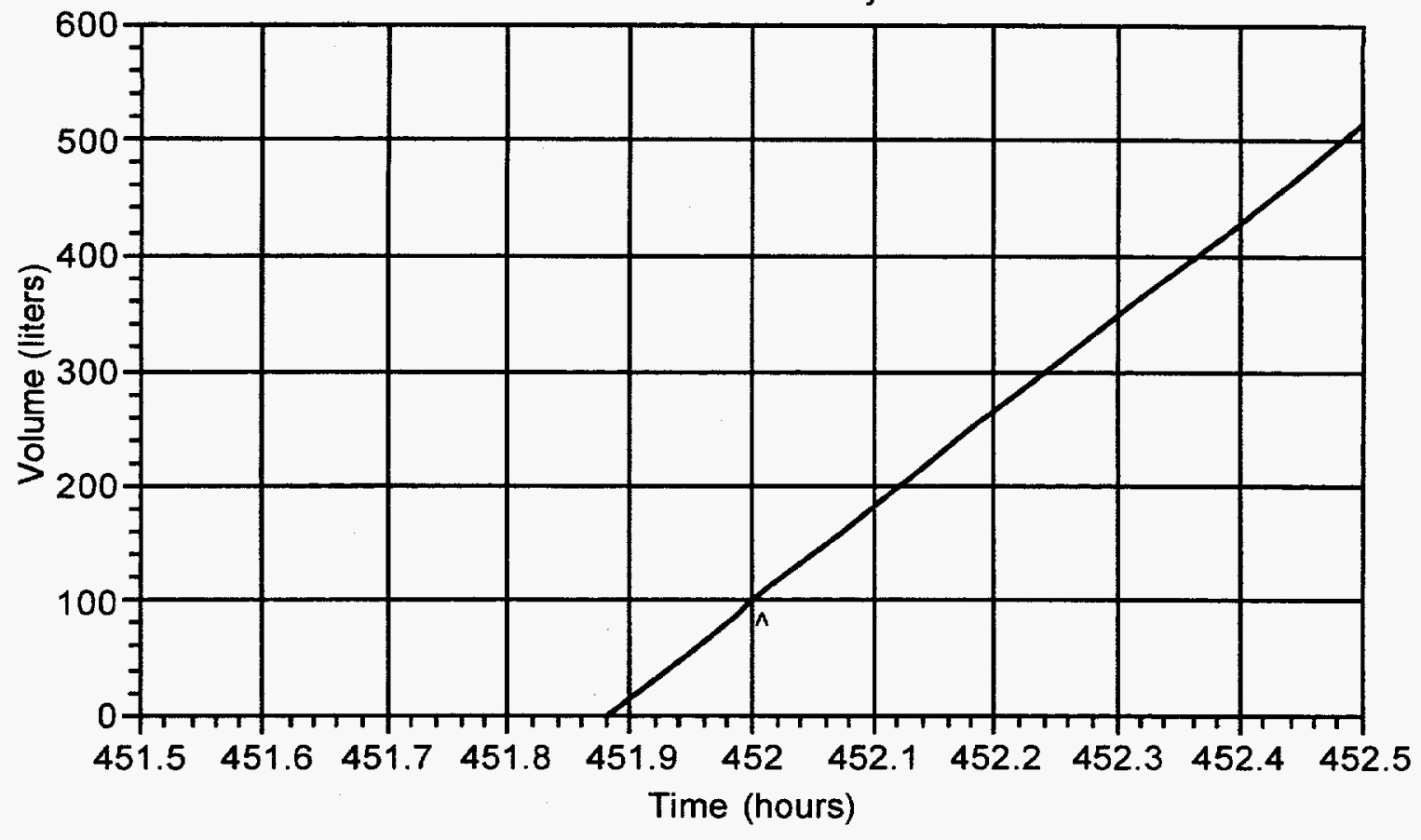

1441 V70 Accountability Vessel

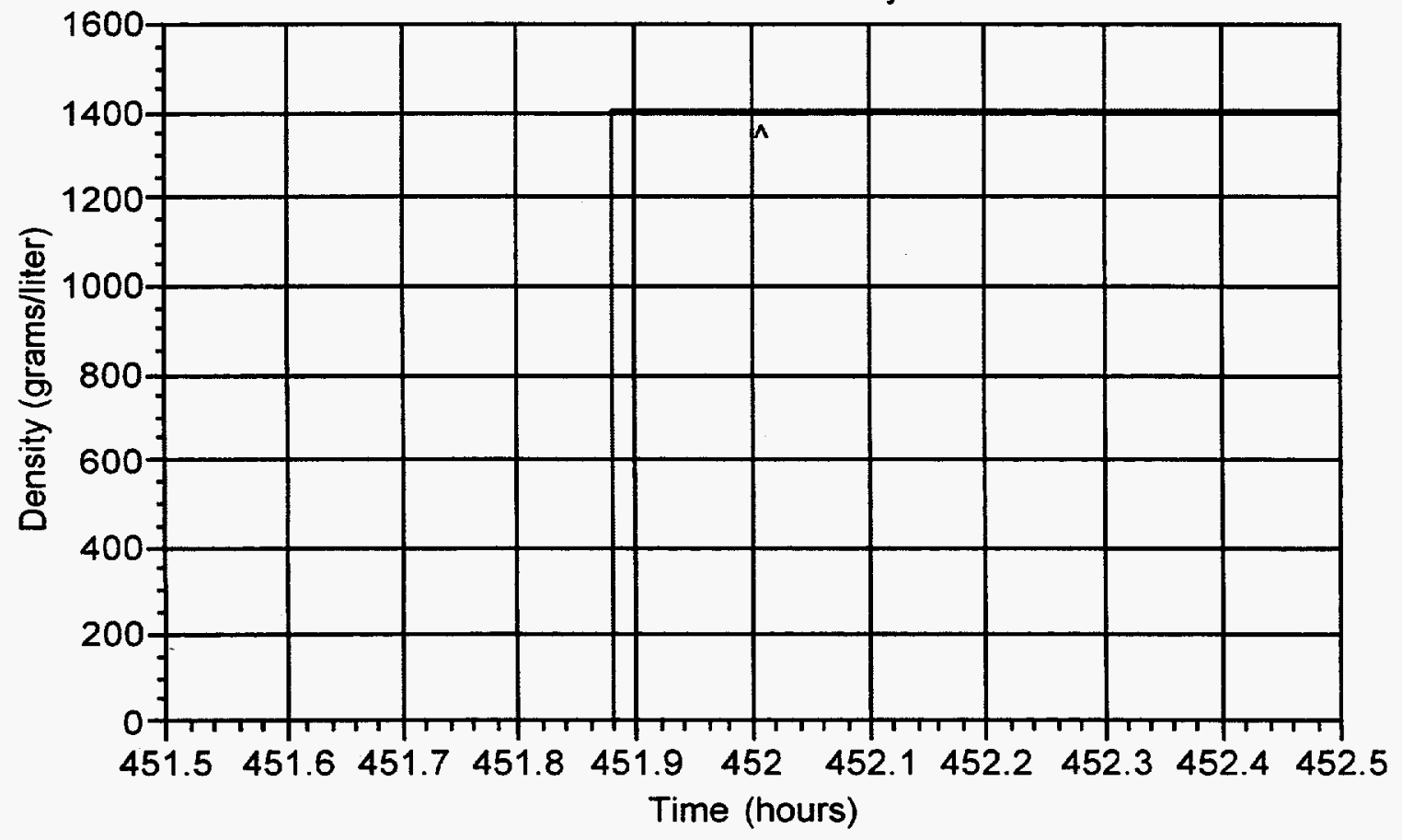

Fig. 16. Case 3a.2. 0.83-liter (0.1\%) diversion without replacement from output accountability vessel 1441 V70 during an input transfer. The diversion began at 451.9873 hours and lasted 5 minutes. 

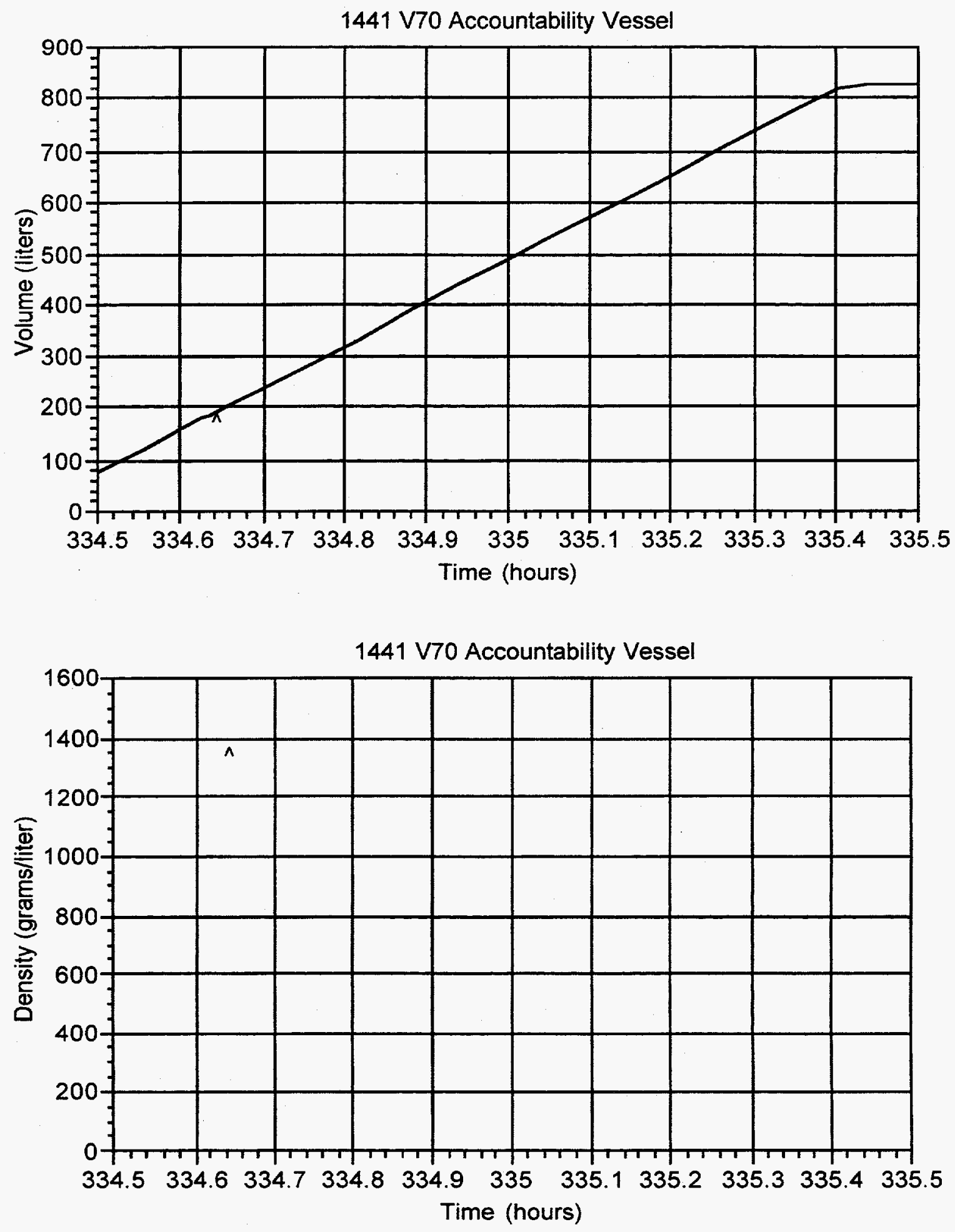

Fig. 16. Case 3b-1. 2.49-liter (0.3\%) diversion without replacement from output accountability vessel 1441 V70 during an input transfer. The diversion began at 334.6362 hours and lasted 5 minutes. 
1421 V71 Buffer Vessel

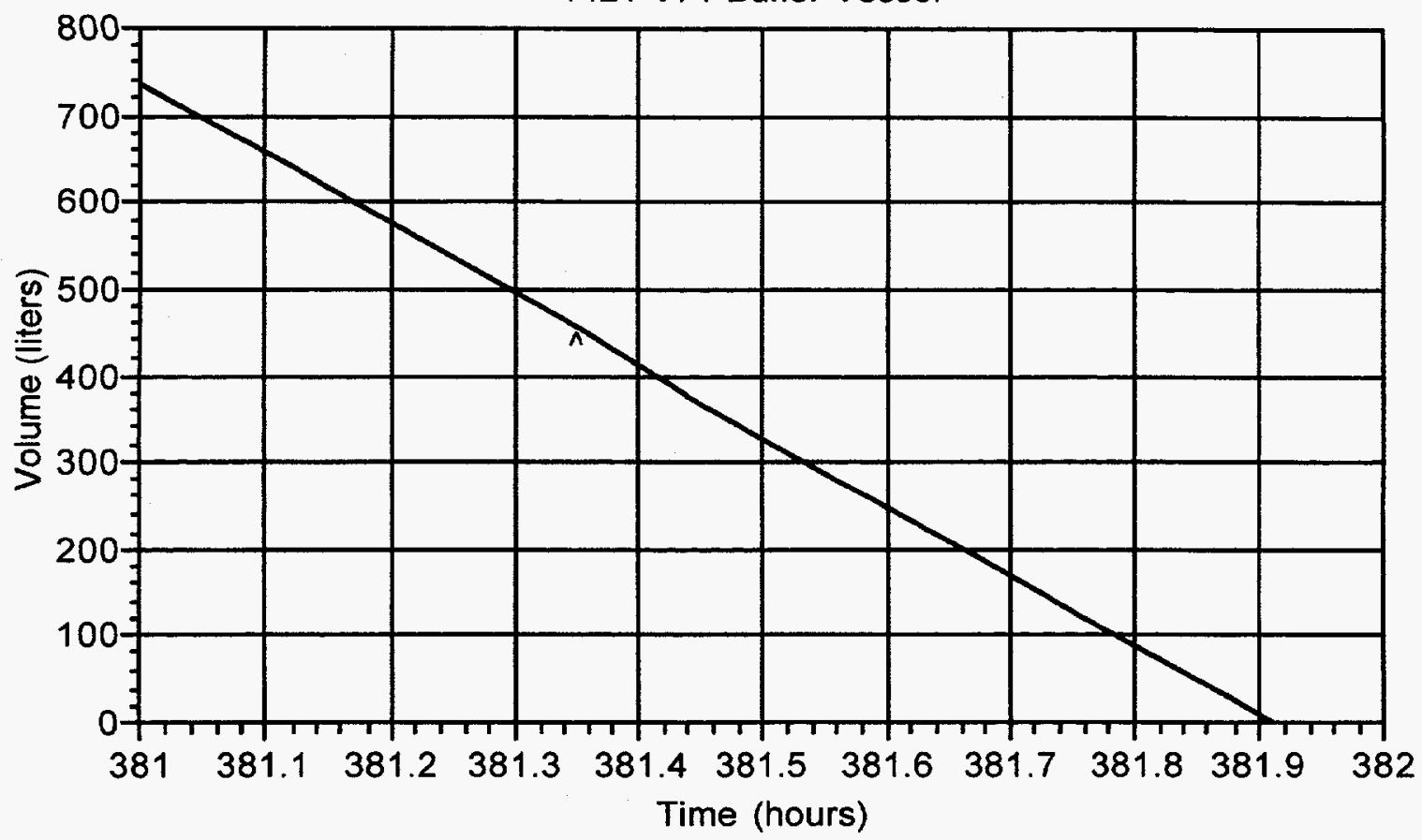

1421 V71 Buffer Vessel

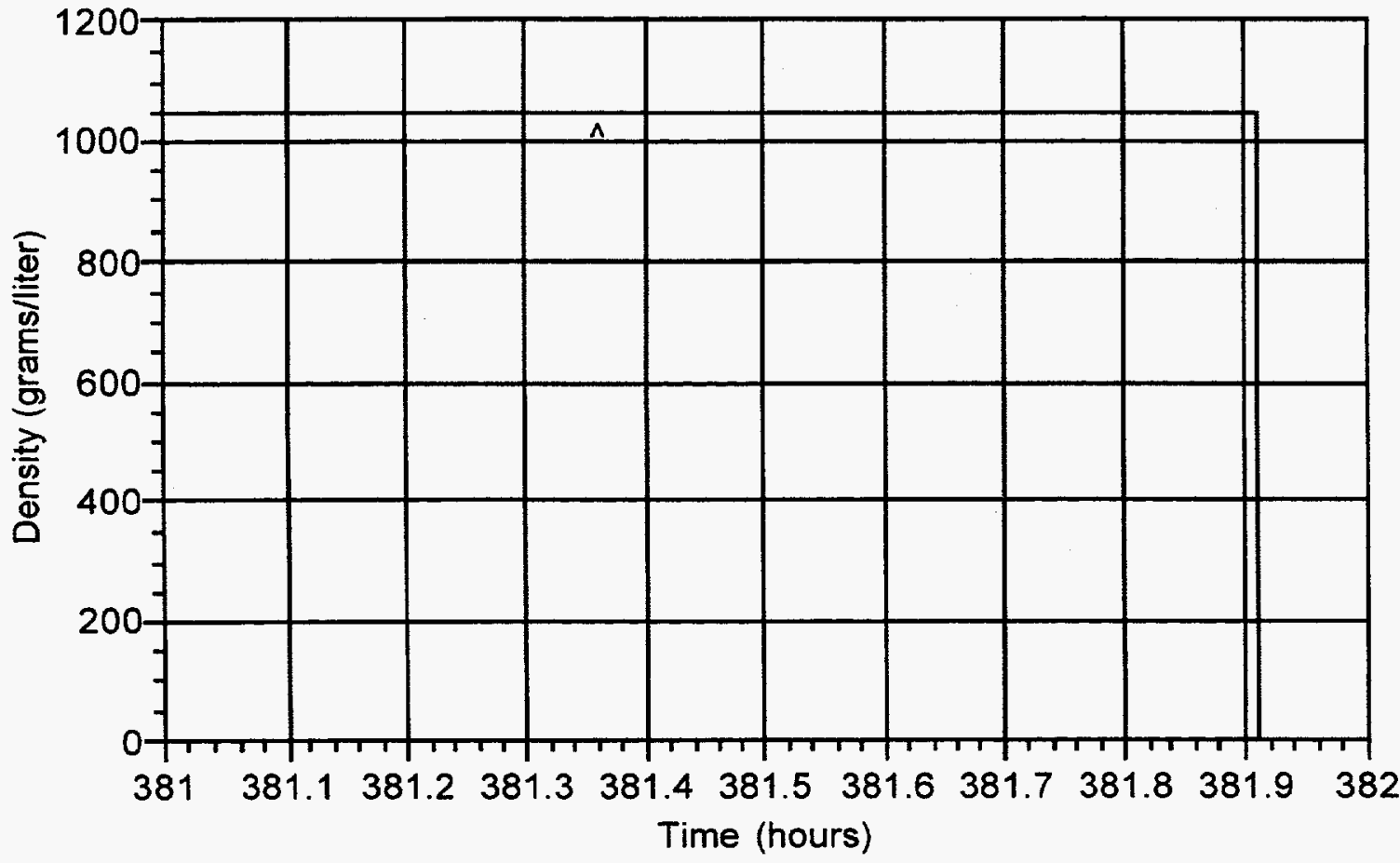

Fig. 18. Case 3c-2. 8.0-liter (1.0\%) diversion without replacement from buffer vessel 1421 V71 during an output transfer. The diversion began at 381.3563 hours and lasted 5 minutes. 
1211 V55 Control Vessel

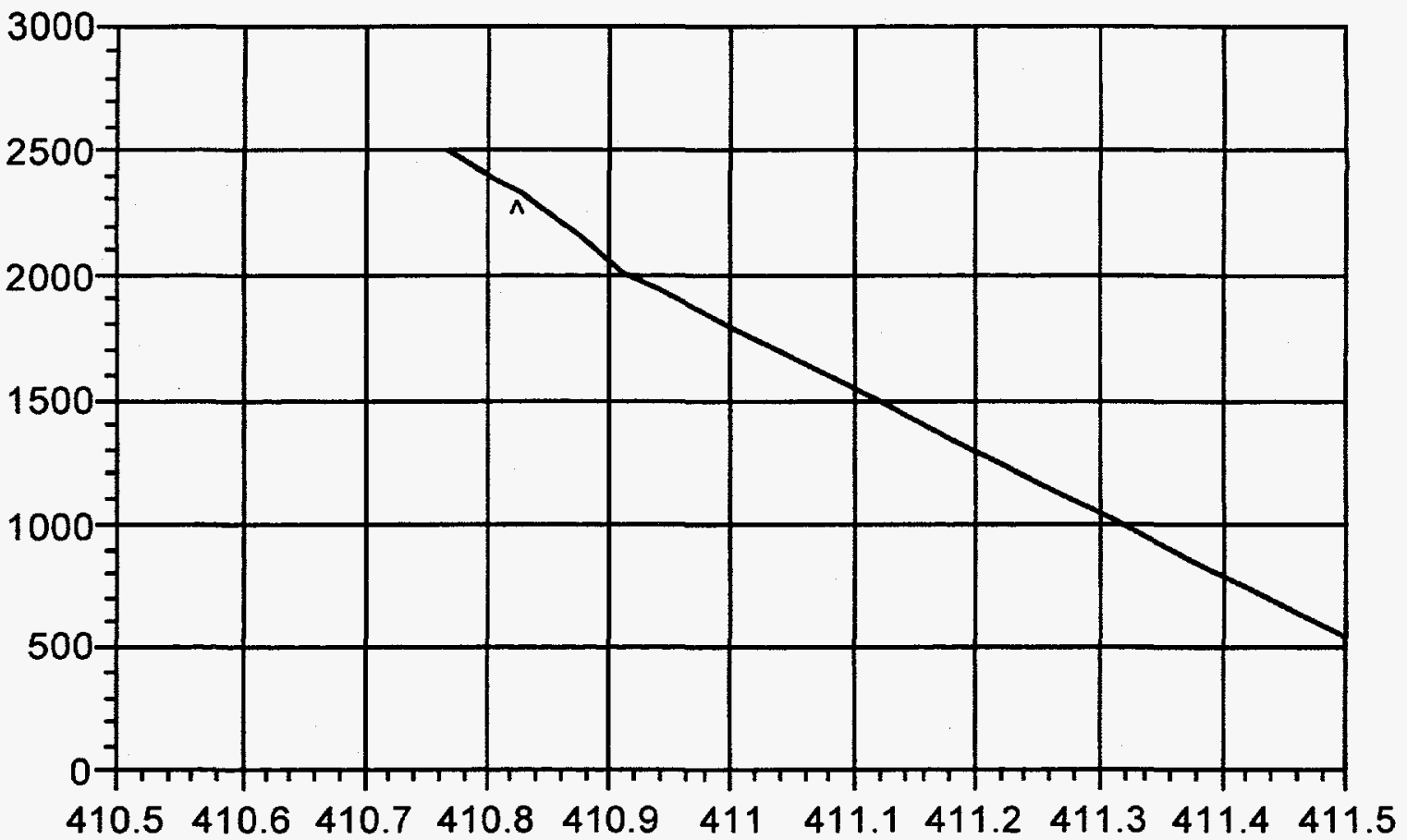

1211 V55 Control Vessel

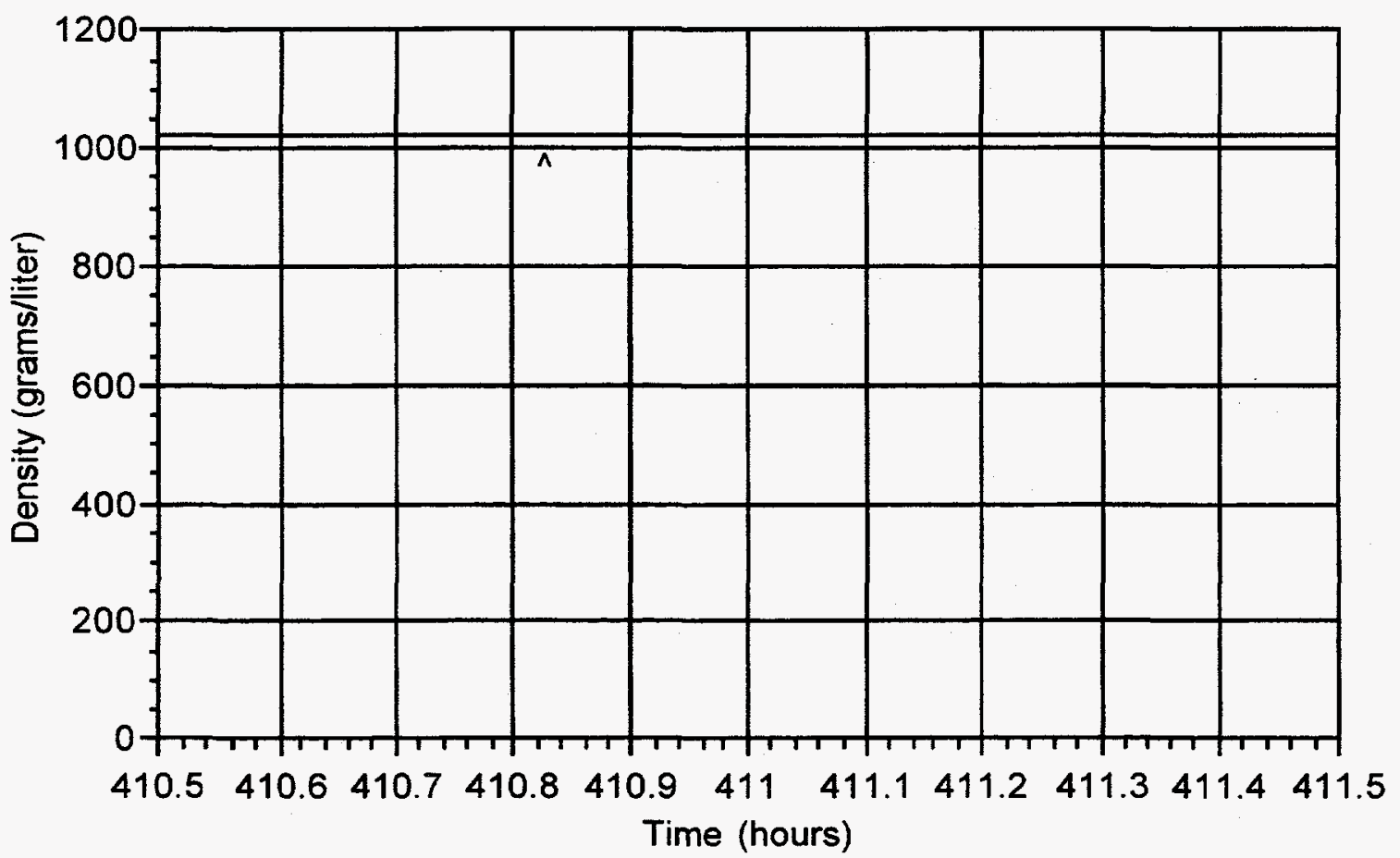

Fig. 19. Case 3d-2. 125-liter (5.0\%) diversion without replacement from control vessel 1211 V55 during an output transfer. The diversion began at 410.8274 hours and lasted 5 minutes. 


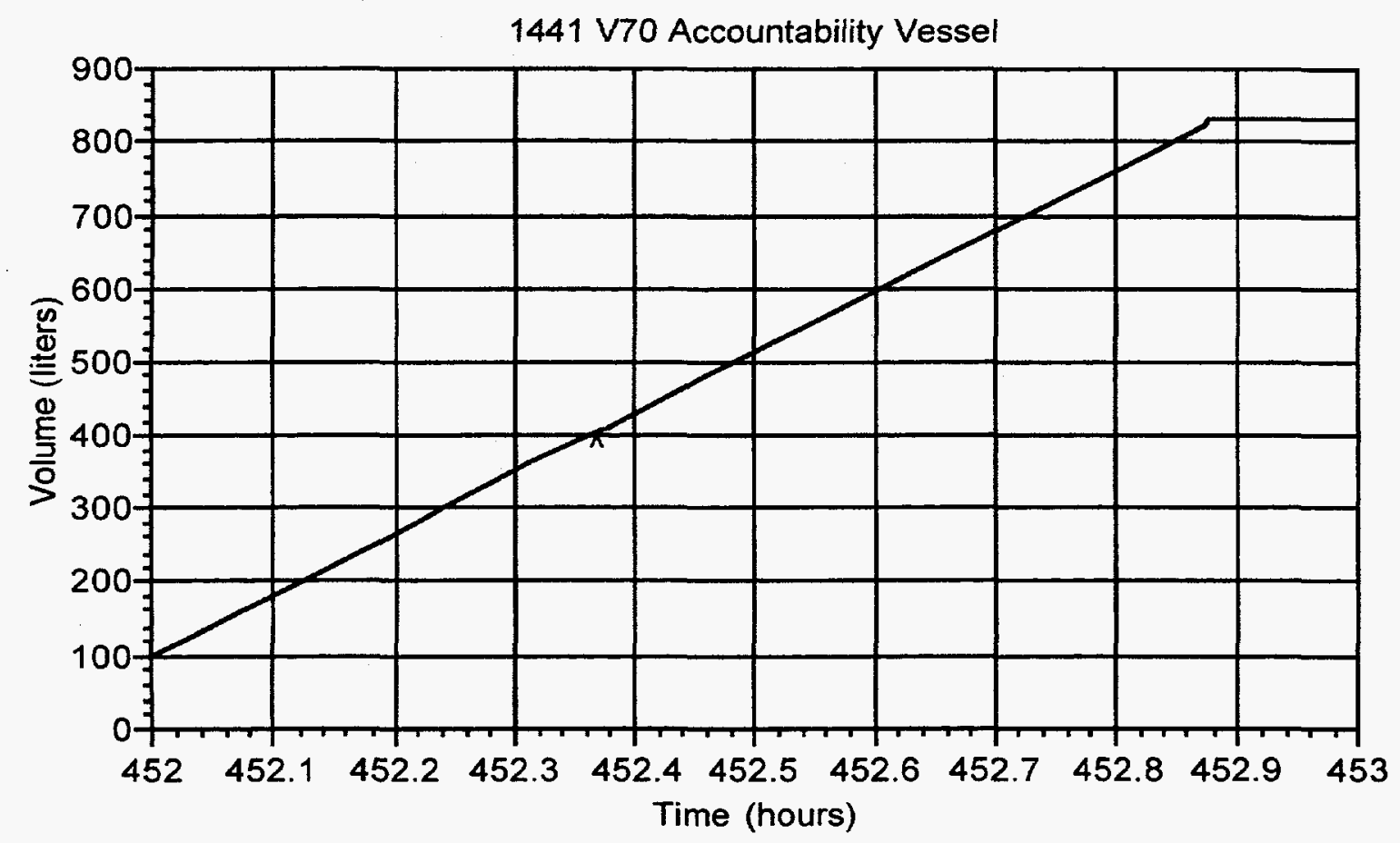

1441 V70 Accountability Vessel

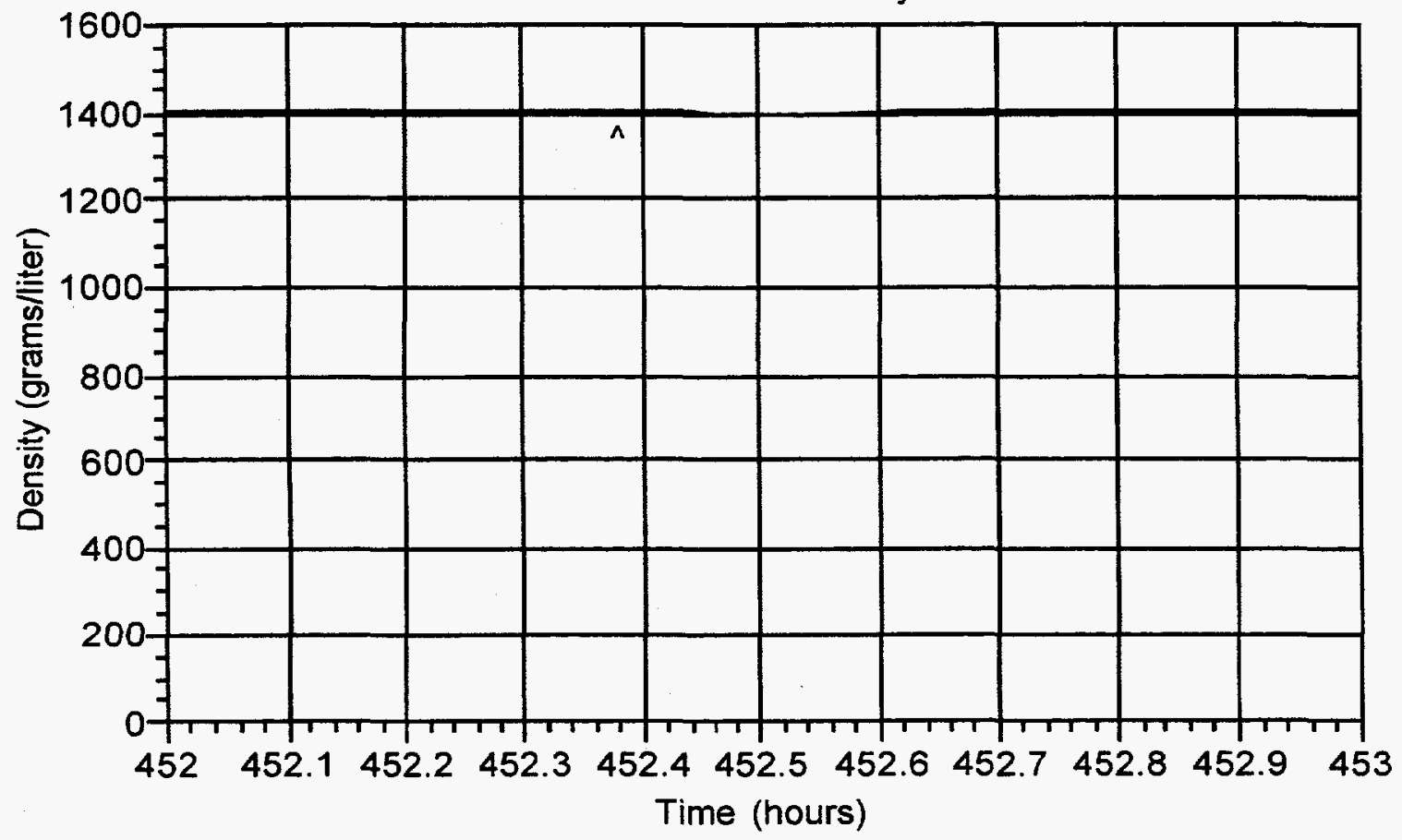

Fig. 20. Case 4a-1 . 0.83-liter (0.1\%) diversion with replacement from output accountability vessel 1441 V70 during an input transfer. The diversion began at 452.3764 hours and lasted 5 minutes. The diverted solution was replaced with an equal volume of 3-molar nitric acid, which caused an initial density decrease of $\sim 0.03 \%$ that was subsequently reduced by half. 
1441 V70 Accountability Vessel

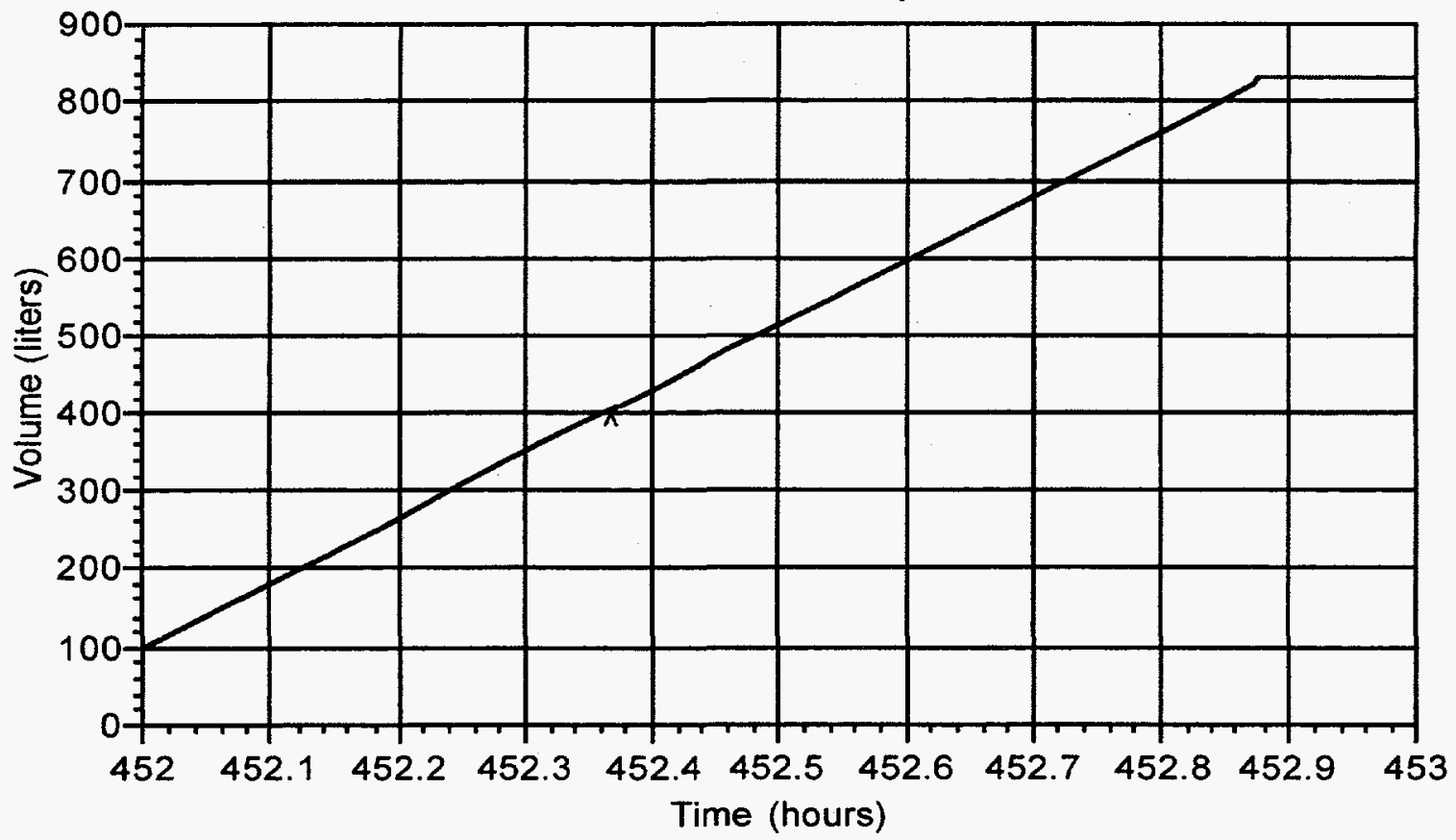

1441 V70 Accountability Vessel

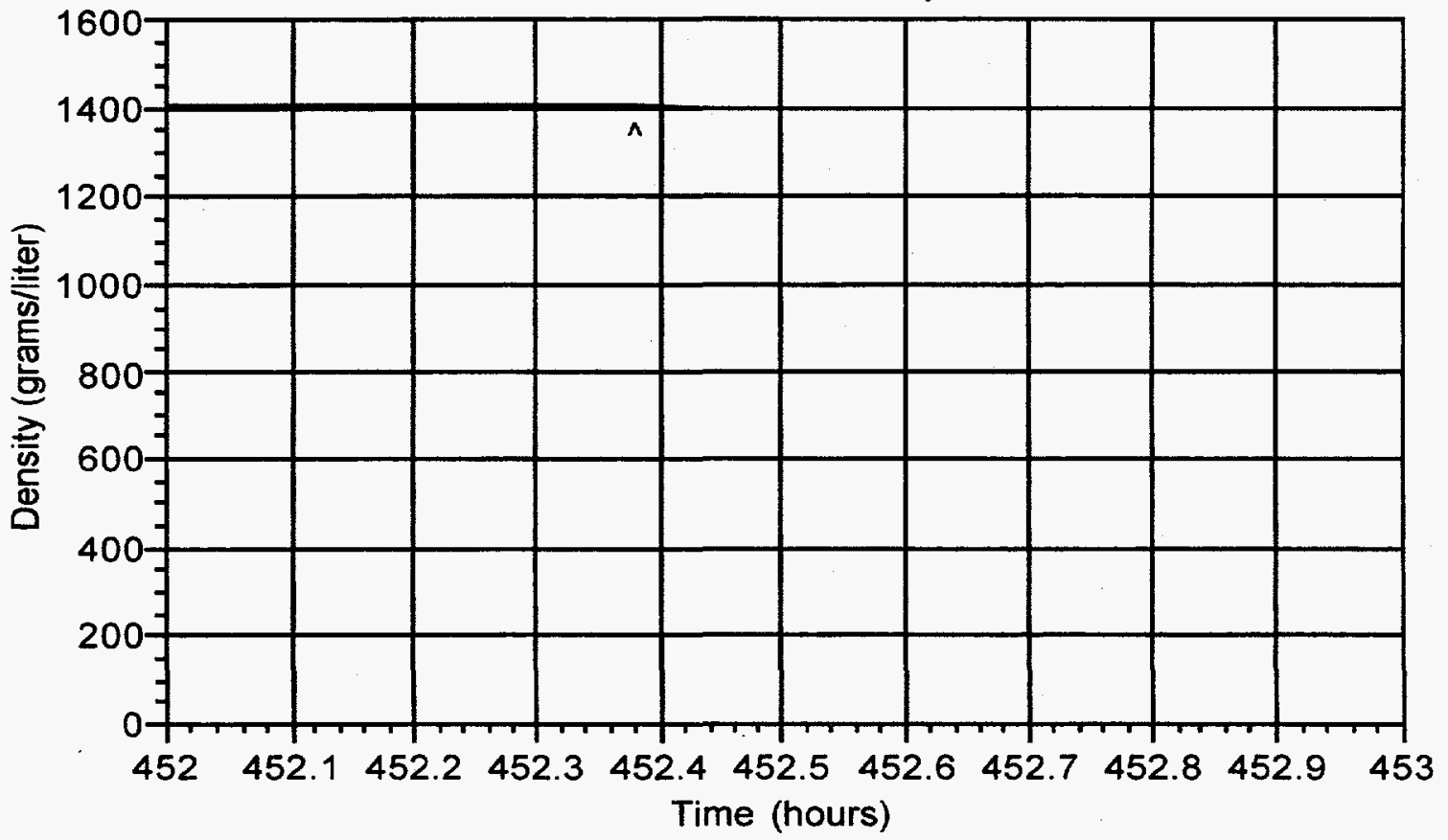

Fig. 21. Case 4b-1. 2.49-liter (0.3\%) diversion with replacement from output accountability vessel 1441 V70 during an input transfer. The diversion began at 452.3764 hours and lasted 5 minutes. The diverted solution was replaced with an equal volume of 3-molar nitric acid, which caused an initial density decrease of $0.1 \%$ that was subsequently reduced by half. 
1421 V73 Control Vessel

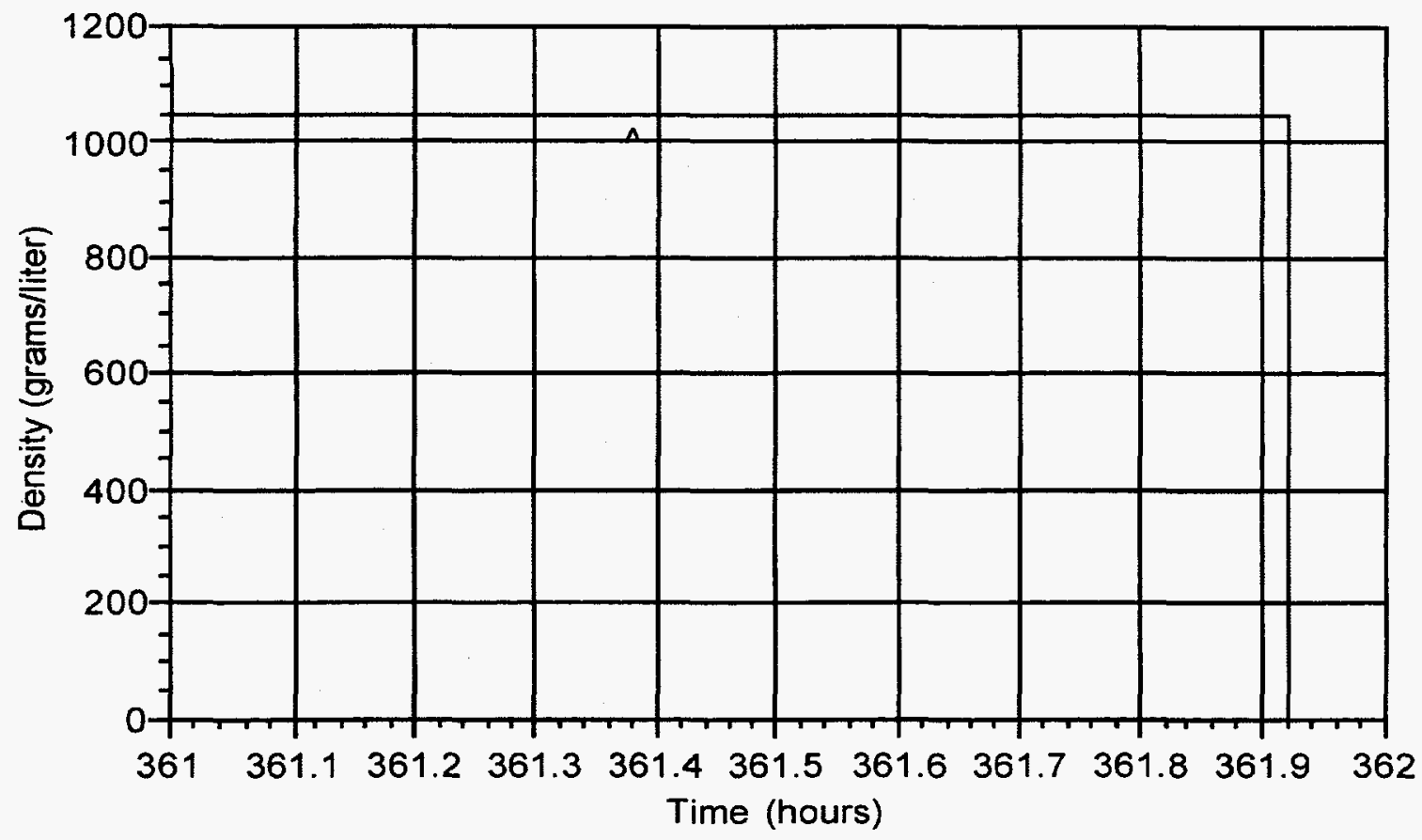

1431 V10 Feeding Vessel

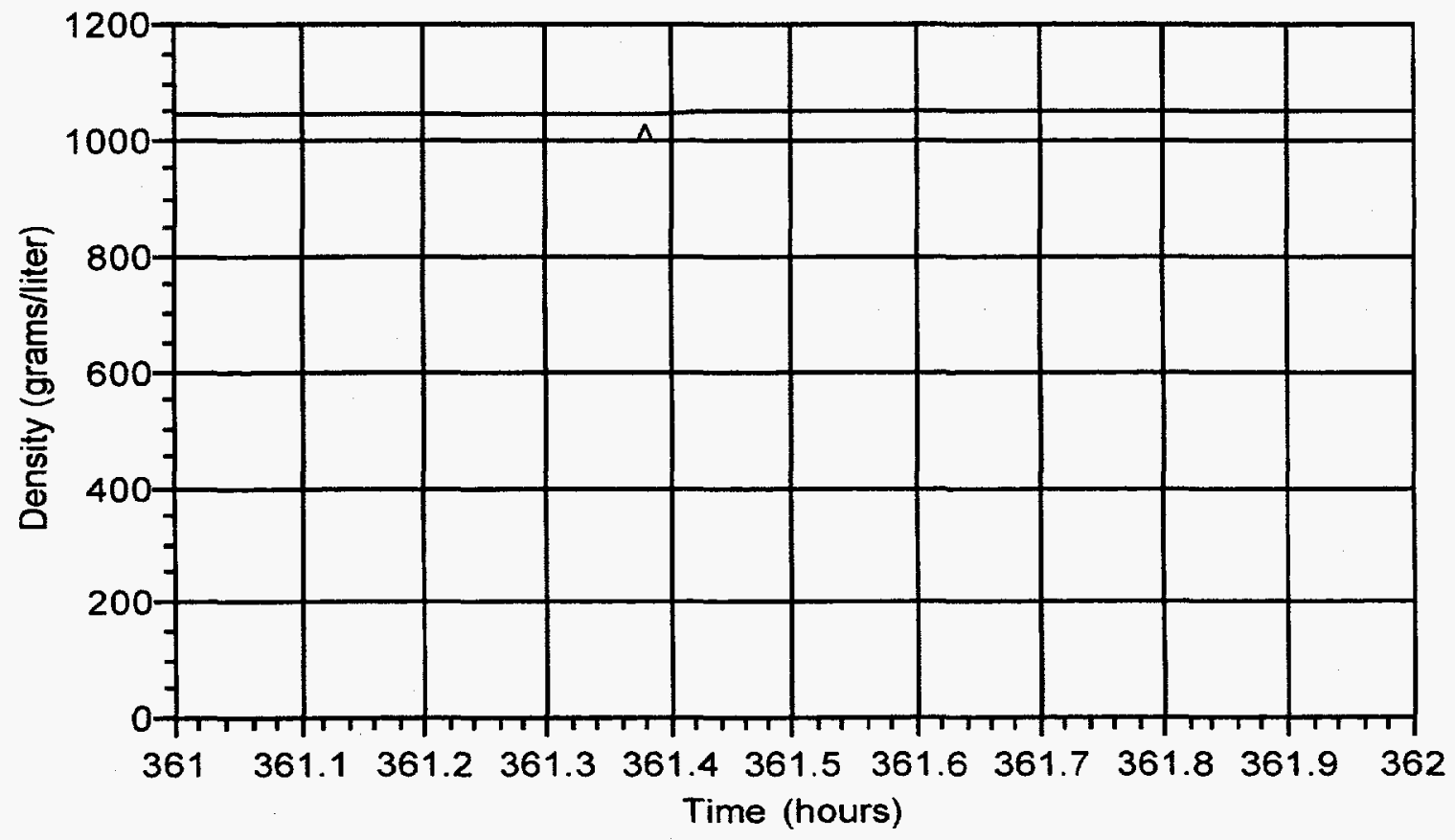

Fig. 22. Case 4c-1. 8.0-liter (1.0\%) diversion with replacement from control vessel 1421 V73 during an output transfer. The diversion began at 361.3847 hours and lasted 5 minutes. The diverted solution was replaced with an equal volume of 3-molar nitric acid, which caused a density increase of $0.1 \%$ in $1421 \mathrm{~V} 73$ and a subsequent $0.03 \%$ increase in density in feeding vessel $1431 \mathrm{~V} 10$ beginning almost immediately. 
1211 V10 Receiving Vessel

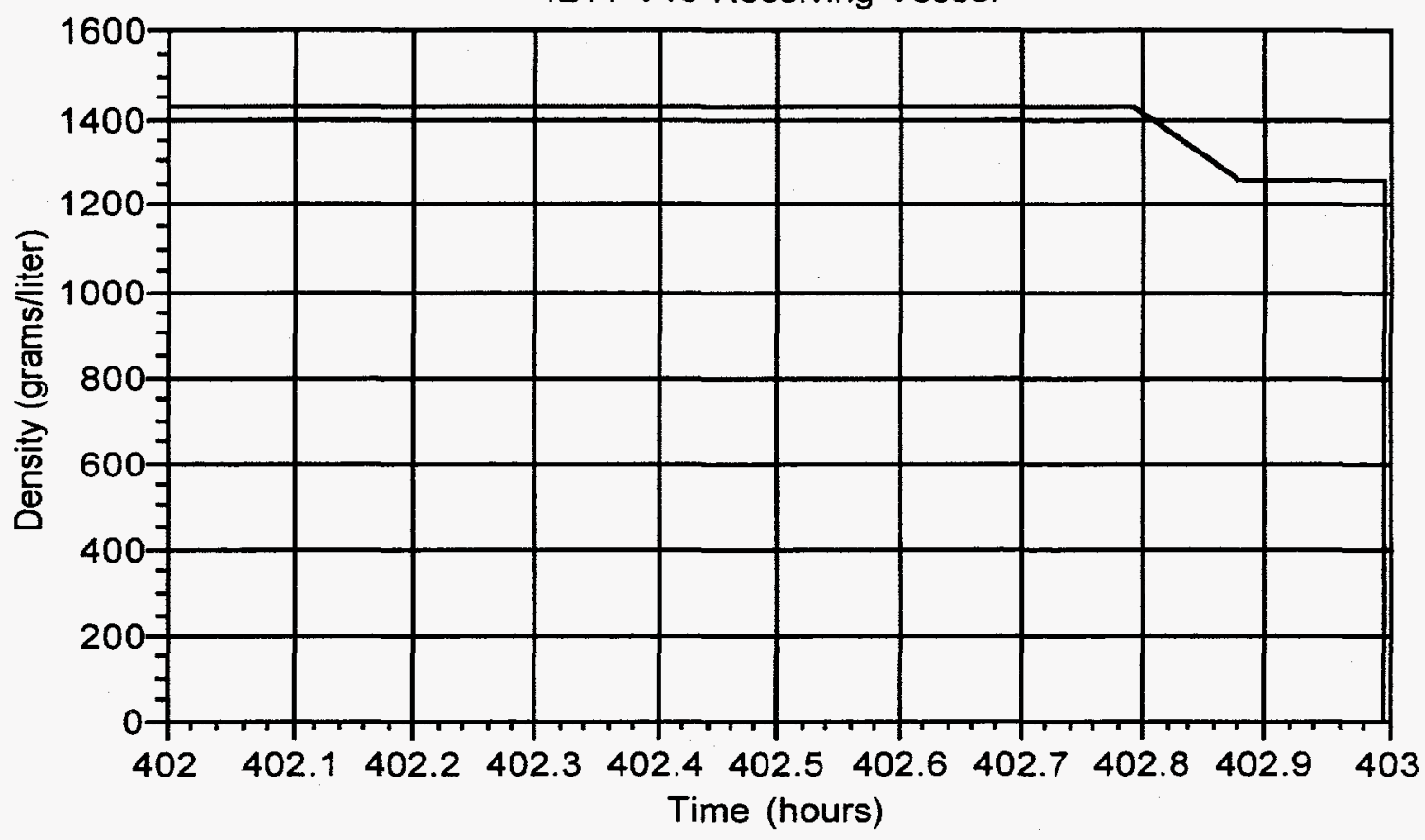

1211 V11 Feeding Vessel

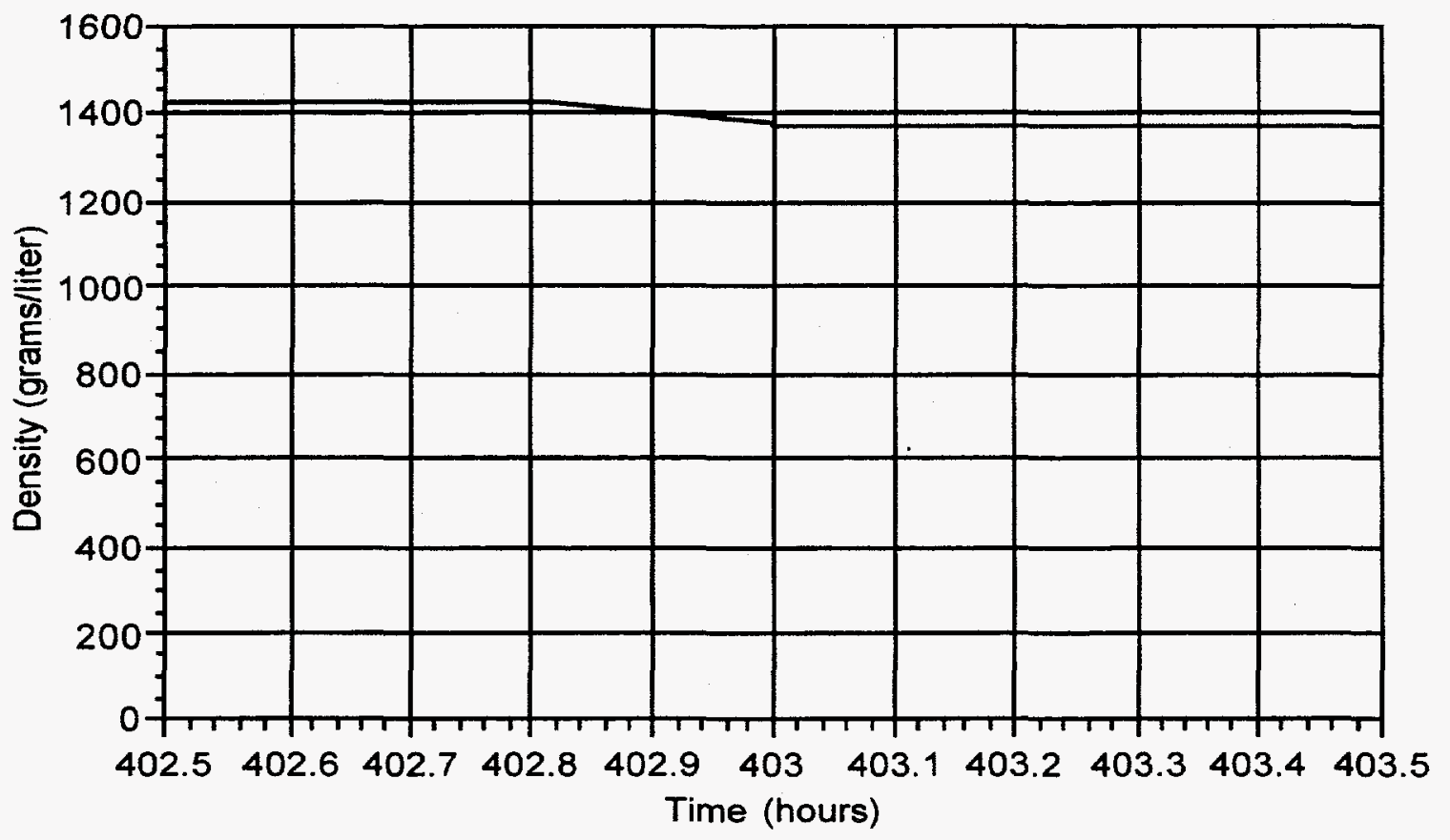

Fig. 23. Case 4d-2a. 981-liter (5.0\%) diversion with replacement from receiving vessel 1211 V10 during an output transfer. The diversion began at 402.7933 hours and lasted 5 minutes. The diverted solution was replaced with an equal volume of 3-molar nitric acid, which caused a density decrease of $11.8 \%$ in $1211 \mathrm{~V} 10$ and a subsequent decrease in density in feeding vessel $1211 \mathrm{~V} 11$ beginning at about 402.8 hours that eventually became as great as $3.5 \%$. 


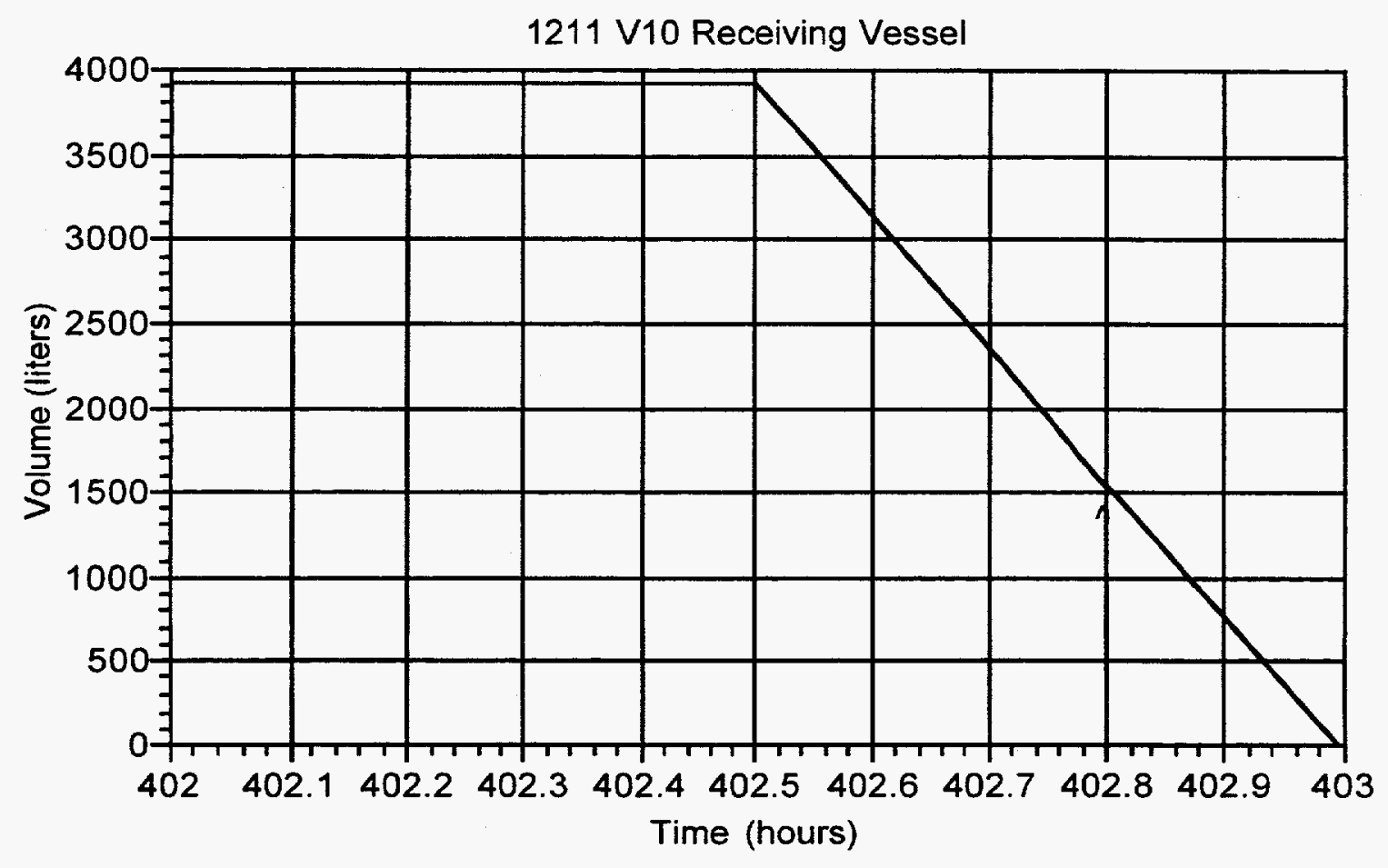

1211 V11 Feeding Vessel

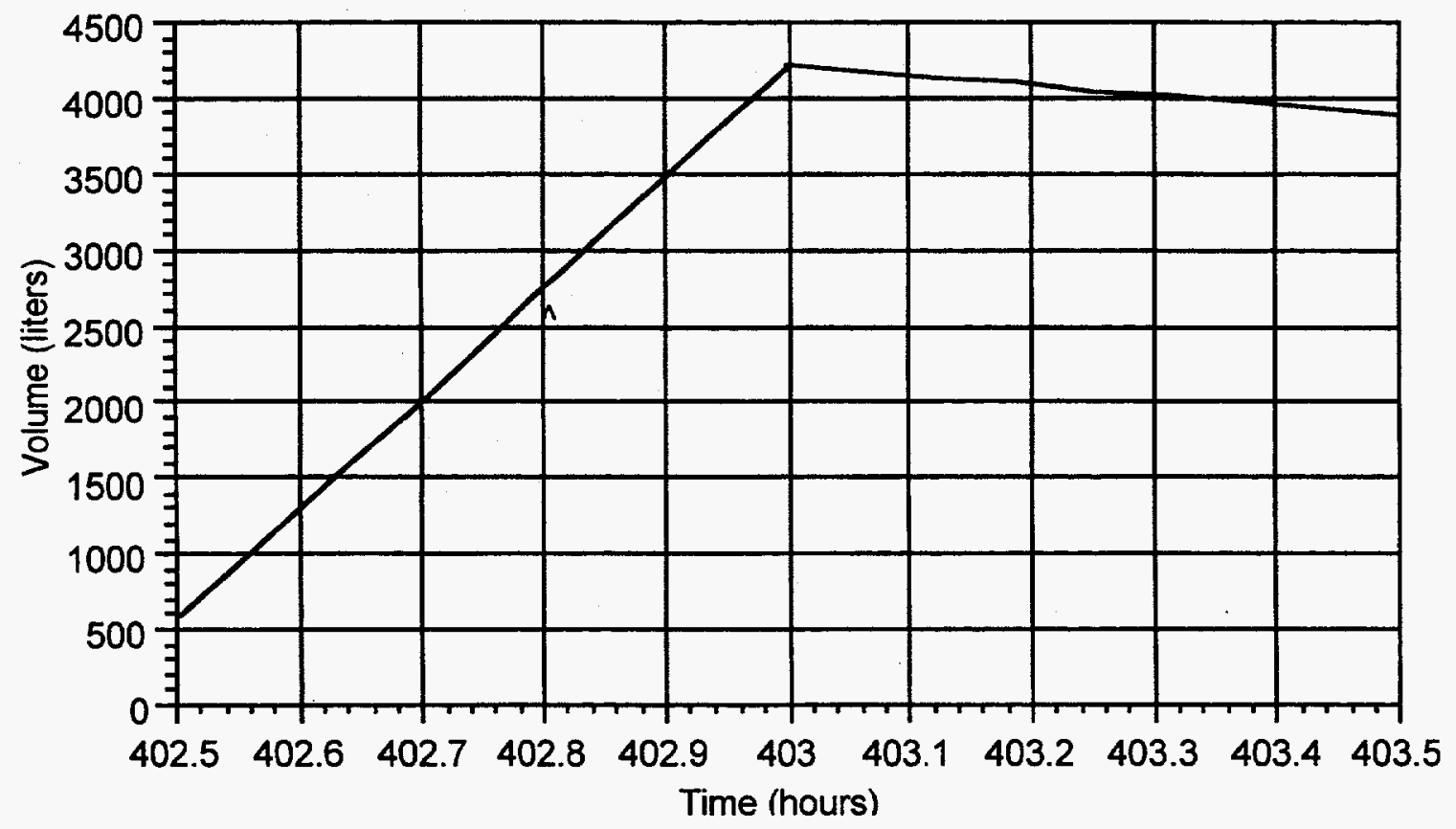

Fig. 24. Case 4d-2b. Volume plots for vessels $1211 \mathrm{~V} 10$ and $1211 \mathrm{~V} 11$ during the diversion with replacement shown in Fig. 23. The large effect of the diversion-with-replacement on the density of the solution in receiving vessel $1211 \mathrm{~V} 10$ was caused by the fact that the vessel, which holds nearly 20,000 liters when full, was nearly empty when the diversion occurred. This is clearly not an optimum diversion strategy. 
1211 R20 Extraction Column

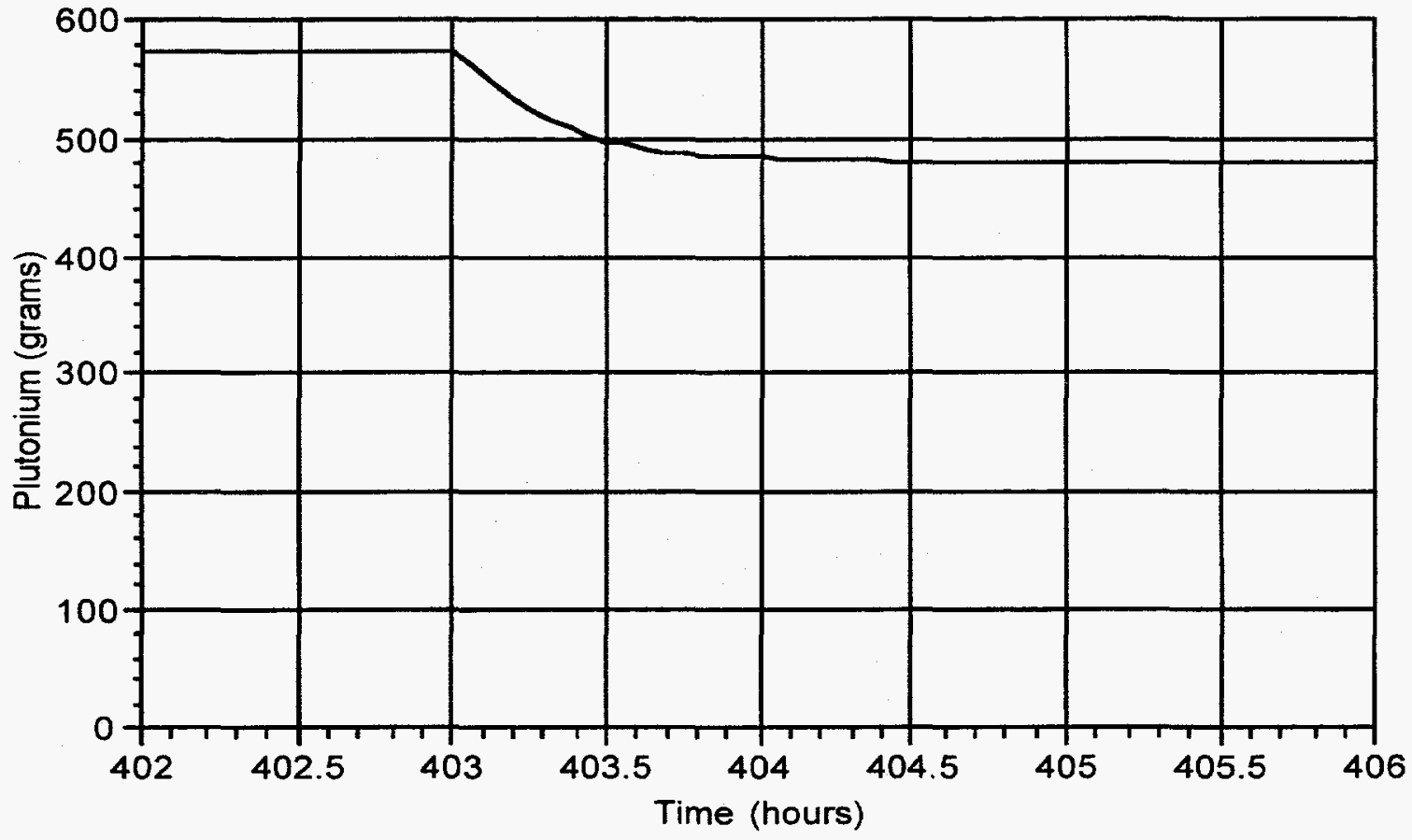

1211 R30 Scrub Column

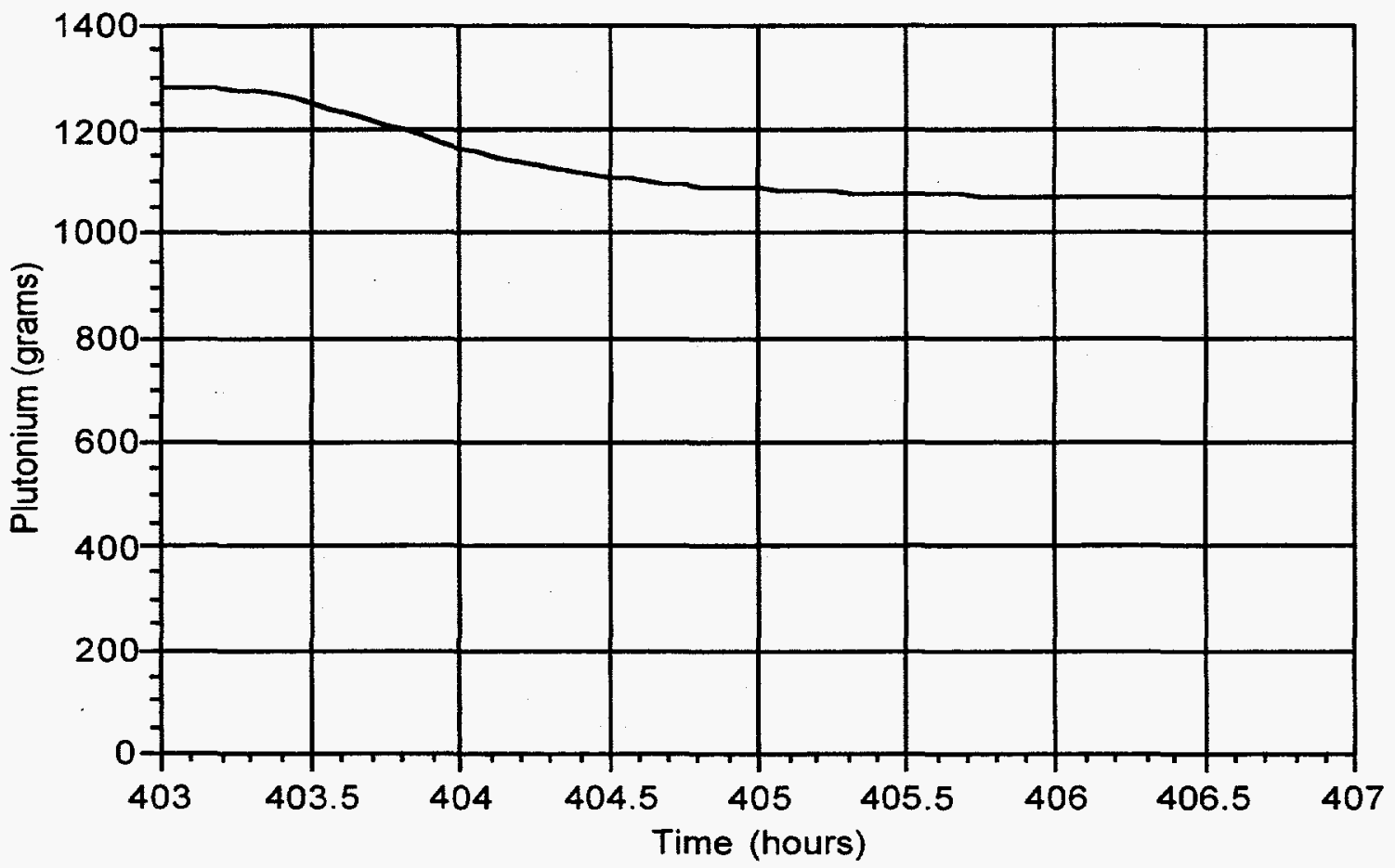

Fig. 25. Case 4d-2c. Plutonium inventory plots for pulsed columns 1211 R20 and 1211 R30 following the diversion with replacement shown in Fig. 23. It requires more than 15 hours for the plutonium inventory of 1211 R20 to return to its pre-diversion value, and more than 25 hours for the plutonium inventory of $1211 \mathrm{R} 30$ to return to its pre-diversion value. 


\subsection{Implications for detectability of diversion strategies}

The simulated diversion scenarios show that a well-designed diversion with replacement, using nitric acid as the replacement solution, can produce negligible changes in solution volume and very small changes in solution density. The detectability of the diversion is further reduced if it occurs during a transfer, because the solution turbulence caused by the declared transfer reduces the accuracy of the level and density measurements and thus their ability to detect undeclared transfers. Diversion with replacement during a declared output transfer may be somewhat easier to detect than diversion with replacement during a declared input transfer, because it may cause nearly simultaneous changes in solution density in two vessels.

It is possible that solution turbulence caused by undeclared solution transfers would produce detectable fluctuations in solution level and density measurements; such fluctuations were not included in the current simulations. Otherwise, it may be necessary to supplement level and density measurements with chemistry measurements in order to achieve acceptable detection sensitivity for solution-diversion-with-replacement scenarios.

\section{Analyses}

\subsection{General approach to the analyses}

The analyses are grouped broadly into two groups: (1) find and identify all tank events (classify each event as a transfer or sample event in the current simulated data), and (2) archive all events so that a given event can be compared to either the historical distribution of such events or to the expected distribution based on measurement error models obtained from tank calibration data. For example, if we find a tank 1 to tank 2 transfer, we compute both the volume and mass shipperreceiver difference (SRD). We compare those SRDs to either the historical SRDs or to the expected distribution of SRDs based on measurement error models obtained from tank calibration data. More detail is given in [3], but the idea is simply that each $(\mathrm{L}, \mathrm{D}, \mathrm{T})$ reading for a given tank is labeled as ship mode, receive mode, or wait mode. Ship modes are further divided into ship-toother-tank mode or ship-to-sample mode. The start and stop volume for a given tank's shipment is recorded so that the shipped volume and mass can be estimated. The same is done for the receiver tank. For the results to be presented here, we assume that error models are known. In practice, this is feasible only after analysis of several months of SM data and facility measurement control and calibration data. If we have good estimates of the true volume and density (and therefore also mass) error models, we can monitor each tank for volume or mass loss. Decision thresholds to identify suspicious events can be used as they are in ordinary quality-control charts. More elaborate quality-control schemes such as multivariate control charts should also eventually be considered. 


\subsection{Data expected}

Instrumentation is expected to include dip tubes for measuring density and level, and temperature gauges. There would be considerable benefit to include an in-tank Pu concentration measuring instrument, but inline Pu concentration measurement will not be assumed here. Concerning the frequency of measurements, see [4], which suggests that the measurement frequency be the minimum of the time required to transfer $8 \mathrm{~kg}$ of $\mathrm{Pu}$ and the time required to allow at least 10 snapshots during a typical tank transfer. Applying that suggestion to RRP, we expect to need level, density, and temperature (L,D,T) measurements every 1-3 minutes. Example simulated L data (in arbitrary units) at approximately 4-minute intervals from steady-state operation of the first 6 main tanks at RRP is shown in Fig. 26. Tank 1 is the input accountability tank. Tank 4 ships to the first set of pulsed columns (where we do not expect SM to be possible), and tank 5 receives from the first set of pulsed columns.

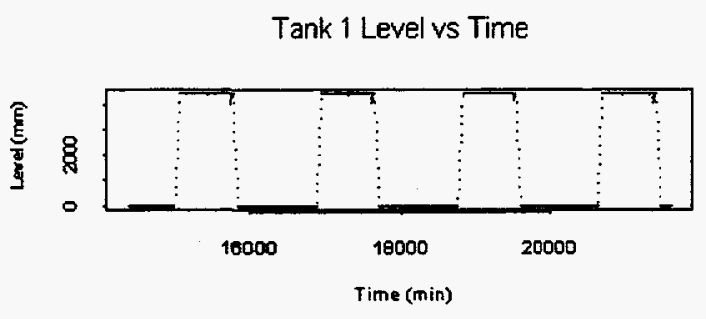

Tank 3 Level vs Time

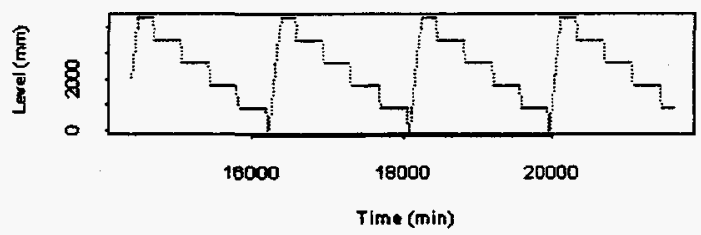

Tank 5 Level vs Time

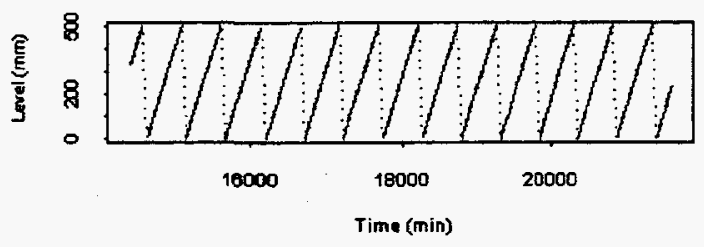

Tank 2 Level vs Time

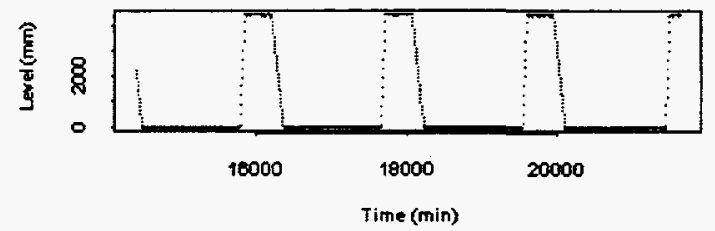

Tank 4 Level vs Time

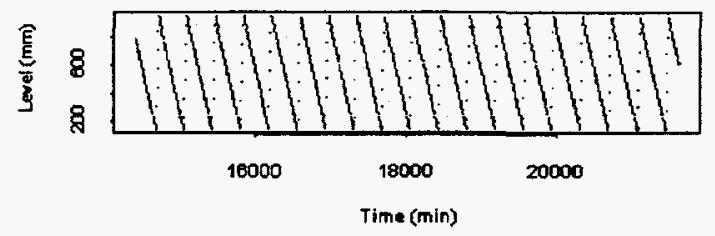

Tank 6 Level vs Time

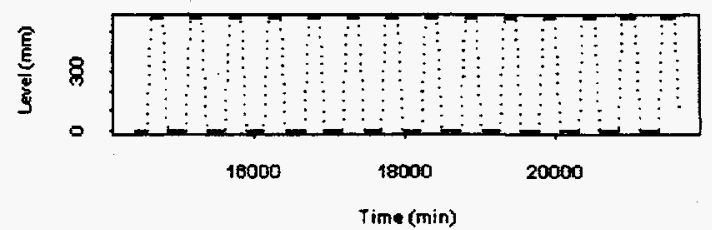

Fig. 26. Approximately 4 days of level readings at $\sim 4$ minute intervals for the first 6 tanks. 


\subsection{Measurement error modeling}

The usual detection goals imply that the monthly $\sigma_{\mathrm{MB}}$ be $0.36 \%$ or less of monthly throughput, and the yearly $\sigma_{\mathrm{MB}}$ be $0.03 \%$ or less of the yearly throughput (assuming an $8000 \mathrm{~kg} /$ year Pu throughput). The monthly detection goal is marginally achievable with good measurements and the yearly goal is not achievable with any conceivable measurements. However, much of the $\sigma_{\mathrm{MB}}$ is due to systematic errors (especially of the volume measurement), and SM can in some cases reduce the effect of systematic errors, as we will show, provided that bias correction methods are effective. It should be noted that a key reference [5] considered the effect of analyzing frequent material balances (MBs) over small material balance areas (individual tanks in our case), and concluded that in the worst-case protracted diversion scenario, less frequent MBs over a single material balance area actually have higher detection probability. The proof used the Neyman Pearson lemma from classical statistics and assumed that the diversion was optimally (from the diverter's view) allocated. Reference [5] did not consider the possibility of performing bias corrections, so the results of this paper complement those in [5].

Any statement about loss detection ability depends critically on the assumed measurement error models. Throughout this paper, we assume the total measurement error variance, $\sigma_{T}^{2}=\sigma_{S}^{2}+\sigma_{R}^{2}$, is the sum of the systematic and random error variances, and that both $\sigma_{S}$ and $\sigma_{R}$ are $0.1 \%$ relative (except where stated otherwise, and we also add $0.1 \%$ additive errors, but they have negligible effect here, so effectively we assume all error models are relative). We discuss only volume and density measurements here and consider testing for volume and/or mass loss.

For volume measurement (and similarly for density measurement), our simple error models (only the relative components will be discussed here) can be written

$$
\mathrm{V}_{\mathrm{i}}=\mathrm{V}_{\mathrm{Ti}}+\mathrm{V}_{\mathrm{Ti}} * S+\mathrm{V}_{\mathrm{Ti}} * R_{\mathrm{i}},
$$

where $\mathrm{V}_{\mathrm{Ti}}$ is the true volume, $S \cong \mathrm{N}\left(0, \sigma^{2}\right)$, and $R-\mathrm{N}\left(0, \sigma_{\mathrm{R}}^{2}\right)$. We assume the systematic error $S$ is constant for a given tank within a calibration period, whereas the random error $R_{\mathrm{i}}$ varies with each measured volume. Error model (1) has two implications that are important here:

(1) the systematic error in the measured receipt by tank 2 will equal the systematic error in the measured shipment by tank 2 , so when we sum the volume shipper-receiver differences (VSRDs), the systematic error variances for all tanks except the first and last are eliminated; and

(2) the systematic error $S$ is constant within a calibration period for a given tank, so bias corrections are feasible.

Equation (1) implies that we can write the $i^{\text {th }}$ VSRD (let $\mathrm{X}_{\mathrm{i}}=\mathrm{VSRD}_{\mathrm{i}}$ ) from tank 1 to tank 2 as

$$
X_{i}=V_{1, i}-V_{2, i}=V_{T}\left(S_{1}-S_{2}+R_{1 i}-R_{2 i}\right) \text {, }
$$


where $\mathrm{V}_{\mathrm{T}}$ is the true shipped volume. Now consider using the average of $n$ of the VSRD $\mathrm{V}_{\mathrm{i}}$ (in the case that all the $\mathrm{V}_{\mathrm{T}_{\mathrm{i}}}$ are the same):

$$
\bar{X}_{n}=V_{T}\left(S_{1}-S_{2}+\bar{R}_{1}-\bar{R}_{2}\right)
$$

to bias-correct future transfers, so

$$
\tilde{X}_{i}=X_{i}-\bar{X}_{n}
$$

will have the same random error variance as $\mathrm{VSRD}_{\mathrm{i}}$ and will have systematic error variance equal to $\mathrm{V}_{\mathrm{T}}^{2}\left(\sigma_{\mathrm{R} 1}^{2}+\sigma_{\mathrm{R} 2}^{2}\right) / \mathrm{n}$ because $R_{1}-R_{2}-\mathrm{N}\left(0, \mathrm{~V}_{\mathrm{T}}{ }^{2}\left(\sigma_{\mathrm{R} 1}^{2}+\sigma_{\mathrm{R} 2}^{2}\right) / \mathrm{n}\right)[6]$.

We can now see why the impact of bias corrections will depend on the relative sizes of $\sigma_{\mathrm{S}}$ and $\sigma_{\mathrm{R} .}$ The number of transfers $n$ that are assumed to have zero true volume loss also impacts the reduction in systematic error variance.

At this stage, it would be premature to consider more complicated error models, but there is no guarantee that our error models will be close enough to reality so that our conclusions will hold on real data. Formally, our conclusions only apply to SM data that follow our assumed error models.

\subsection{Estimated loss detection probabilities}

We expect about 15 main process tanks at RRP (the first 9 are referred to in Fig. 26). Seven of the tanks operate in batch-ship and batch-receipt mode (B/B) and the other eight tanks operate in either batch-ship and continuous-receipt or vice versa $(\mathrm{B} / \mathrm{C}$ or $\mathrm{C} / \mathrm{B})$ modes. The tanks that feed the pulsed columns ship continuously and the tanks that receive from the pulsed columns receive continuously. The same is true for the tanks that feed or receive from the evaporator. One technical issue will involve the measurement of shipments or receipts by tanks in $\mathrm{B} / \mathrm{C}$ or $\mathrm{C} / \mathrm{B}$ mode. We divide this as follows.

First, we will simplify the discussion and consider only a 3-tank problem having all B/B tanks, such as the first 3 tanks in Fig. 26, and we will only consider the ability to detect a volume loss, $\mathrm{V}_{8 \mathrm{gkg}}$, or mass loss $\mathrm{M}_{8 \mathrm{~kg}}$, that would equate to a loss of $8 \mathrm{~kg}$ of Pu. For example, if the $\mathrm{Pu}$ concentration in tank 1 were $2.5 \mathrm{~g} / \mathrm{L}$, then $\mathrm{V}_{8 \mathrm{~kg}}=8000 / 2.5=3200 \mathrm{~L}$. We will then compare the 3-tank case to the more realistic 15-tank case for detecting a loss of $8 \mathrm{~kg}$. Second, we consider only the 15-tank system with losses such as those described in section 2 (the FacSim section). A "volume loss" case means that volume was removed from the system. A "mass loss" case means that volume was conserved by replacement using lower-than-nominal-density solution, so the mass loss was detected. 
3.4.1. Three-tank system, $\mathbf{V}_{\mathbf{8 k g}}$ and $\mathbf{M}_{\mathbf{8 k g}}$ cases. Tank 1 ships to tank 2 , which ships to tank 3. In this section, we assume that software such as that described in [3] and [6] has been used to locate all shipments and receipts by all three tanks during one 200-day "year." We can then divide all tank snapshots ( $L, D, T$ readings) into two categories for a given tank: wait mode or transfer mode. Having done that, in this section, we consider four SM activities:

(1) Monitor each of the three tanks for volume loss during all wait modes. "Wait mode" means that no abrupt level changes occurred, though there could have been a slow evaporation or other event that slowly affected the level.

(2) Monitor each of the 2 shipper-receiver pairs for volume loss during all transfer modes.

(3) and (4) Same as 1 and 2, but for mass.

Note that unless we have an in-line Pu concentration measurement, we cannot completely monitor these three tanks. Because we do expect to at least have a density measurement, we can monitor the three tanks for loss of mass, as we will describe. That means that any statistically detectable diversion would have to be concealed by replacing the lost mass with proper density solution. What we accomplish is that the adversary must work hard to conceal the diversion (and would likely be discovered at the time that Pu was measured off-line because of out-ofspecification chemical species). The same type of calculations would apply if we used an in-line $\mathrm{Pu}$ concentration measurement, and in that case there would be no way for the adversary to conceal a statistically detectable diversion.

Monitoring wait mode is easier than monitoring transfer mode because of the impact of systematic errors that cancel for a given tank during a wait mode and that do not cancel during transfer modes. This means that the optimal diversion time (from the diverter's view) is during transfers. The reason is that during a wait mode, we need only check for a significant change in volume or mass. If some legitimate but long-term event like evaporation is occurring, then the volume will decrease, but the density will increase, so that the mass will remain approximately constant. (Actually, evaporation causes the mass to decrease slightly because some evaporate is removed, and this source of variability in the estimated mass loss would have to be investigated.)

As a reference case, consider a typical NRTA approach where we compare the total volume shipped by tank 1 to that shipped by tank 3 over one 200-day year of operation. Assume the following:

- input batches to tank 1 take 1 hour and occur once every 32 hours: $20,000 \mathrm{~L}$ at $2.5 \mathrm{~g} / \mathrm{L}$ (approximately 154 batch shipments per 200 day "year"), and same for batch shipments to tank 2 (154 shipments per year), and

- output batches from tank 3 occur once every 6.4 hours: $4000 \mathrm{~L}$ at $2.5 \mathrm{~g} / \mathrm{L}$ (770 shipments per year). 
Fix the overall false-alarm probability at $\alpha=0.05$ and calculate the failure-to-detect probability, $\beta$, of detecting $8 \mathrm{~kg}$ of the worst-case protracted diversion scenario (over one year) considered in [7]. To account for multiple testing, apply the Bonferroni [8] correction to the individual $\alpha$ 's $\left(\alpha_{i}=0.05 / 2^{3}\right.$ to account for separate tests for mass and volume, separate tests for wait or transfer modes, and separate tests for abrupt or protracted diversion). Though the reference case only tests for loss during transfer modes, the SM cases also test for loss during wait modes, so we elected to include a correction for testing both modes. For volume loss, $\beta=0.97$ and for mass loss, $\beta=0.99$ (so $\beta$ is much higher [i.e., worse] than the goal of $\beta=0.05$ ). To compute $\beta$, we first compute $S=$ (volume [or mass] needed) $/ \sigma$ where $\sigma$ is the measurement-error standard deviation of the total measured volume (or mass) shipped by tank 1 minus that for tank 3 . $S=0.66$ for volume, and $S=0.47$ for mass in our reference case. We then compute the $\beta$ as the probability that a $N(0,1)$ variable is less than $T-S$, where $T$ is the $1-\alpha_{i}$ quantile of the $N(0,1)$ distribution. so we see that $\beta$ will be very large, even if we do not correct for multiple testing (i.e., if we let $\left.\alpha_{i}=\alpha\right)$.

3.4.1.1. Protracted loss using SM data. As in [7] and our reference case, we consider only the worst case (unrealistic but useful to consider) protracted diversion scenario, so it can be shown that we should sum the measured volume (or mass) losses over all wait modes over all tanks (and do the same for transfer modes). Fortunately, systematic errors cancel for the wait modes (because we consider changes in the same tank). Unfortunately, systematic errors do not cancel for the transfer modes, but they do cancel for all tanks except the first and last. The biascorrected systematic errors also do not cancel for the transfer modes, but the hope is that they will be smaller (in the sense of having smaller variance) than the non-bias-corrected systematic errors. In all cases below (Table 2), we report $\beta$ for fixed $\alpha=0.05$ (so $\alpha_{i}=0.05 / 8$ to correct for testing for both wait or transfer, abrupt or protracted, and mass or volume) for detecting a loss of $8 \mathrm{~kg}$ both with and without bias corrections (BC) using $n=.2 *$ nships (nships is the number of shipments per year, which is 154 for both shipper-receiver pairs), $n=.2 *$ nships, and $n=.5 *$ nships as the number of transfers $n$ to average in Eq. (3).

In Table 2, we see the impact of the number of transfers $n$ used to estimate the bias correction: for volume loss, $\beta$ is $0.35,0.16,0.06$, respectively, as $n$ equals 0.1 to 0.2 to 0.5 times the number of shipments per year, and for mass loss, $\beta$ is $0.47,0.31$, and 0.21 , respectively. Table 2 suggests that protracted loss detection in a three-tank system with all $\mathrm{B} / \mathrm{B}$ mode tanks would greatly benefit from bias corrections available from SM data. 
Table 2. Tanks 1-3, BC Results Given for $n=0.1 * n s h i p s$, $n=0.2 *$ nships, $n=0.5 *$ nships.

\begin{tabular}{|l|c|c|}
\hline \multicolumn{1}{|c|}{ Activity } & $\beta($ no $\mathrm{BC})$ & $\beta(\mathrm{BC})$ \\
\hline 1. V loss during wait modes & 0.06 & 0.06 \\
\hline 2. V loss during transfer modes & 0.97 & $0.35,0.16,0.06$ \\
\hline 3. M loss during wait modes & 0.37 & 0.37 \\
\hline 4. M loss during transfer modes & 0.97 & $0.47,0.31,0.21$ \\
\hline
\end{tabular}

3.4.1.2. Abrupt loss using SM data. One good reason to use SM data is the greatly improved abrupt-loss detection. We will define abrupt loss to be a loss that occurs over either a single wait mode for one tank or over a single shipment from one tank to another. To correct for multiple testing, again we first use $\alpha_{i}=0.05 / 8$, and then also divide by the number of tanks and the number of wait or transfer modes as appropriate. Then, when we consider detecting the needed volume or mass to divert $8 \mathrm{~kg}$ of Pu, during either the wait or transfer modes (of the three $\mathrm{B} / \mathrm{B}$ modes tanks), we have $\alpha<0.01$ for all three tanks, which is excellent loss-detection ability. So, abrupt-loss detection is expected to be excellent without bias corrections.

3.4.2. 15-tank system, $V_{8 \mathrm{~kg}}$ and $M_{8 \mathrm{~kg}}$ cases. We now consider the 15 -tank system. As a reference case, consider a typical NRTA approach where we compare the total volume or mass shipped by tank 1 to that shipped by tank 15 over one 200-day year of operation (though it is not meaningful to compare the volume shipped by tank 1 to that shipped by tank 15 , we will do so for a reference case). Assume:

- same assumptions for tank 1 as in section 3.4.1, and

- output batches from tank 15 occur once every 120 hours: $830 \mathrm{~L}$ at $0.25 \mathrm{~kg} / \mathrm{L}$ (40 shipments per year).

Fix the false-alarm probability at $\alpha=0.05$ as we do throughout this report, and calculate the failure-to-detect probability, $\beta$, of $8 \mathrm{~kg}$ of the worst-case protracted diversion scenario (over one year) considered in [7]. For volume loss, $\beta=0.97$ and for mass loss, $\beta=0.98$ (so $\beta$ is much higher than the goal of $\beta=0.05$ ). A minor complication arises in calculating the volume needed to divert $8 \mathrm{~kg}$ because the Pu per volume differs among tanks. We convert measurement error variances in each tank to what they would be for a reference tank (tank 1), having fixed Pu concentration to circumvent this complication.

3.4.2.1. Protracted loss using SM data. Increasing from 3 to 15 tanks will increase $\beta$ for both the wait modes and the transfer modes. The increase in $\beta$ for the wait modes is simply because there are more wait modes when there are more tanks. The increase in $\beta$ for the transfer modes is also partly because there are more tanks and also because some tanks operate in $\mathrm{B} / \mathrm{C}$ or $\mathrm{C} / \mathrm{B}$ mode. 
The random error variance for a VSRD from a C/B tank like tank 14 to a B/B tank like tank 15 is larger than if tank 14 were in B/B mode, because the V shipped by tank 14 is more difficult to calculate (tank 14 is receiving while it is shipping). To model this increase, we let $\sigma_{\mathrm{R}}$ vary from $0.1 \%$ to $0.2 \%$ to $0.5 \%$ relative in Tables $3-5$ below.

Table 3. Tanks $1-15, \sigma_{R}=0.1 \%$ relative, $B C$ results for $n=0.1 * n s h i p s$, 0.2*nships, $0.5 *$ nships.

\begin{tabular}{|l|c|c|}
\hline \multicolumn{1}{|c|}{ Activity } & $\beta($ no BC) & $\beta(\mathrm{BC})$ \\
\hline 1. V loss during wait modes & 0.40 & 0.40 \\
\hline 2. V loss during transfer modes & 0.98 & $0.84,0.81,0.73$ \\
\hline 3. M loss during wait modes & 0.71 & 0.71 \\
\hline 4. M loss during transfer modes & 0.98 & $0.93,0.90,0.87$ \\
\hline
\end{tabular}

Table 4. Tanks $1-15, \sigma_{R}=0.2 \%$ relative, $B C$ results for $n=0.1 * n s h i p s$, 0.2*nships, $0.5 *$ nships.

\begin{tabular}{|l|c|c|}
\hline \multicolumn{1}{|c|}{ Activity } & $\beta($ Ho BC) & $\beta(\mathrm{BC})$ \\
\hline 1. V loss during wait modes & 0.40 & 0.40 \\
\hline 2. V loss during transfer modes & 0.98 & $0.95,0.93,0.91$ \\
\hline 3. M loss during wait modes & 0.71 & 0.71 \\
\hline 4. M loss during transfer modes & 0.99 & $0.97,0.96,0.95$ \\
\hline
\end{tabular}

Table 5. Tanks $1-15, \sigma_{R}=0.5 \%$ relative, $B C$ results for $n=0.1 * n s h i p s$, 0.2*nships, $0.5 *$ nships.

\begin{tabular}{|l|c|l|}
\hline & \multicolumn{1}{|c|}{ Activity } & \multicolumn{1}{|c|}{$\boldsymbol{\beta}(\mathrm{BC})$} \\
\hline 1. V loss during wait modes & 0.21 & 0.21 \\
\hline 2. V loss during transfer modes & 0.99 & $0.98,0.98,0.98$ \\
\hline 3. M loss during wait modes & 0.50 & 0.50 \\
\hline 4. M loss during transfer modes & 0.99 & $0.99,0.99,0.98$ \\
\hline
\end{tabular}

In Tables 3-5, we see that loss detection during the transfer modes is difficult, and we do not expect to even come close to the goal of $\beta=0.05$. In fact, we might not improve on the reference case that considered cumulative shipments by tank 1 and tank 15 (the reference case has $\beta=0.97$ for $V$ and $\beta=0.98$ for $M$ ). Both the number of tanks and the presence of $C$-mode tanks increase $\beta$, plus we have to correct for multiple testing (wait modes and transfer modes) that is not present in the reference case. Tables 3-5 suggest that protracted loss detection in a 15-tank system with some $B / C$ or $C / B$ tanks would only slightly benefit from bias corrections available from SM data. However, again we find that abrupt loss detection would be greatly improved, as we show below. 
3.4.2.2. Abrupt loss using SM data. As for the three-tank case, we define abrupt loss to be a loss that occurs over either a single wait mode for one tank or over a single shipment from one tank to another. To correct for multiple testing, again we first use $\alpha_{i}=0.05 / 8$ and then also divide $\alpha$ by the number of tanks and the number of wait or transfer modes as appropriate. Then, when we consider detecting the needed volume or mass to divert $8 \mathrm{~kg}$ of $\mathrm{Pu}$ during the wait modes (of the seven $\mathrm{B} / \mathrm{B}$ mode tanks), we have $\beta<0.01$ for all $7 \mathrm{~B} / \mathrm{B}$ mode tanks, which is excellent loss detection ability. As anticipated, loss detection during the transfer modes of tanks 1-14 is more difficult and will depend on how well the batch transfers can be measured for tanks that have a $\mathrm{C}$ modes. If we assume $\sigma_{\mathrm{R}}=\sigma_{\mathrm{S}}=0.1 \%$ then $\beta$ for tanks $1-14$ is less than 0.01 for both volume and loss. If we increase $\sigma_{R}=\sigma_{S}$ to $0.3 \%$, then $\beta$ is less than 0.01 for both volume and mass loss for all tanks except for mass loss for tank 14 , for which $\beta=0.03$. If we apply bias corrections with $n=0.2 *$ cycles, then all $\beta$ 's for all cases are less than 0.01 . Here, bias corrections are likely to improve loss detection that is already quite good.

3.4.3. 15-tank system, abrupt removal of less than $\mathbf{V}_{\mathbf{8 k g}}$ or $\mathbf{M}_{\mathbf{8 k g}}$ cases. In this section, we present results for the 15-tank system for abrupt volume removals of $\mathrm{V}_{\mathrm{R}}=0.1 \%, 0.3 \%$, $1.0 \%, 5.0 \%$, and $\mathrm{V}_{8 \mathrm{~kg}}$ (and the same for mass removal) for first the wait modes of all $\mathrm{B} / \mathrm{B}$ tanks and then the transfer modes of all tanks. For brevity, we do not consider the bias-corrected versions here. The seven $\mathrm{B} / \mathrm{B}$ tanks are tanks $1,2,3,6,11,12$, and 15 , and $\beta$ is given for these seven tanks in that order in Tables 6-10. For the 14 shipper-receiver pairs, results are presented in the natural order: the first listed is tank 1 to tank 2 , the second is tank 2 to tank 3 , and so on. Recall that the diversion scenarios generated by FacSim were abrupt diversions, so this section applies to those scenarios. In all cases, the overall $\alpha$ is fixed at 0.05 , so the individual $\alpha$ 's are adjusted using the conservative Bonferroni correction. For losses during transfer modes, we consider $\sigma_{\mathrm{R}}=\sigma_{\mathrm{S}}=0.1 \%$ and $0.3 \%$ (probably the $0.3 \%$ is extremely optimistic for $\mathrm{B} / \mathrm{C}$ or $\mathrm{C} / \mathrm{B}$ mode tanks). For losses during wait modes we consider only $\sigma_{R}=\sigma_{S}=0.1 \%$.

Table 6. Abrupt volume loss, $\sigma_{R}=\sigma_{S}=0.1 \%$ during wait modes.

\begin{tabular}{|l|l|}
\hline $\mathrm{V}_{\mathrm{R}}$ & \multicolumn{1}{|c|}{$\beta$ (rounded to nearest 0.1 ) } \\
\hline $0.1 \%$ & 1.0 for all $7 \mathrm{~B} / \mathrm{B}$ tanks \\
\hline $0.3 \%$ & 1.0 for all $7 \mathrm{~B} / \mathrm{B}$ tanks \\
\hline $1.0 \%$ & $0,0,0.4,0,0,0,0$ for the 7 tanks \\
\hline $5.0 \%$ & 0 for all $7 \mathrm{~B} / \mathrm{B}$ tanks \\
\hline $\mathrm{V}_{8 \mathrm{~kg}}$ & 0 for all $7 \mathrm{~B} / \mathrm{B}$ tanks \\
\hline
\end{tabular}


Table 7. Abrupt mass loss, $\sigma_{\mathrm{R}}=\sigma_{\mathrm{S}}=\mathbf{0 . 1 \%}$ during wait modes.

\begin{tabular}{|c|l|}
\hline $\mathrm{M}_{\mathrm{R}}$ & \multicolumn{1}{|c|}{$\beta$ (rounded to nearest 0.1) } \\
\hline $0.1 \%$ & 1.0 for all 7 B/B tanks \\
\hline $0.3 \%$ & 1.0 for all 7 B/B tanks \\
\hline $1.0 \%$ & $\begin{array}{l}0.3,0.3,0.8,0.4,0.3,0.3,0.2 \text { for the } \\
7 \text { tanks }\end{array}$ \\
\hline $5.0 \%$ & 0 for all 7 B/B tanks \\
\hline $\mathrm{V}_{8 \mathrm{gg}}$ & 0 for all 7 B/B tanks \\
\hline
\end{tabular}

Table 8. Abrupt volume loss, $\sigma_{R}=\sigma_{S}=0.1 \%$ during transfer modes.

\begin{tabular}{|c|l|}
\hline $\mathrm{V}_{\mathrm{R}}$ & \multicolumn{1}{|c|}{$\beta$ (rounded to nearest 0.1) } \\
\hline $0.1 \%$ & 1.0 for all 14 pairs of tanks \\
\hline $0.3 \%$ & 1.0 for all 14 pairs of tanks \\
\hline $1.0 \%$ & $\begin{array}{l}0.3,0.3,0.3,0.1,0.4,0.4,0,1,0.1,0.4, \\
0.4,1,0,0.2\end{array}$ \\
\hline $5.0 \%$ & 0 for all 14 pairs of tanks \\
\hline $\mathrm{V}_{8 \mathrm{~kg}}$ & 0 for all 14 pairs of tanks \\
\hline
\end{tabular}

Table 9. Abrupt mass loss, $\sigma_{\mathrm{R}}=\sigma_{\mathrm{S}}=\mathbf{0 . 1 \%}$ during transfer modes.

\begin{tabular}{|c|l|}
\hline $\mathrm{M}_{R}$ & \multicolumn{1}{|c|}{$\beta$ (rounded to nearest 0.1) } \\
\hline $0.1 \%$ & 1.0 for all 14 pairs of tanks \\
\hline $0.3 \%$ & 1.0 for all 14 pairs of tanks \\
\hline $1.0 \%$ & $\begin{array}{l}0.3,0.8,0.2,0.5,0.3,0.9,0.3,1,0.6, \\
0.3,0.4,1,0.4,0.8\end{array}$ \\
\hline $5.0 \%$ & 0 for all 14 pairs of tanks \\
\hline $\mathrm{V}_{8 \mathrm{~kg}}$ & 0 for all 14 pairs of tanks \\
\hline
\end{tabular}

Table 10. Abrupt volume loss, $\sigma_{R}=\sigma_{S}=0.3 \%$ during transfer modes.

\begin{tabular}{|c|l|}
\hline $\mathrm{V}_{\mathrm{R}}$ & \multicolumn{1}{|c|}{$\beta$ (rounded to nearest 0.1 ) } \\
\hline $0.1 \%$ & 1.0 for all 14 pairs of tanks \\
\hline $0.3 \%$ & 1.0 for all 14 pairs of tanks \\
\hline $1.0 \%$ & $0.3,0.3,0.4,1,1,1,1,1,1,1,0.4,1,1,1$ \\
\hline $5.0 \%$ & 0 except tank 8: 1, tank 12:0.5 \\
\hline $\mathrm{V}_{8 \mathrm{~kg}}$ & 0 for all 14 pairs of tanks \\
\hline
\end{tabular}


Table 11. Abrupt mass loss, $\sigma_{R}=\sigma_{s}=0.3 \%$ during transfer modes.

\begin{tabular}{|c|l|}
\hline $\mathrm{M}_{\mathrm{R}}$ & \multicolumn{1}{|c|}{$\beta$ (rounded to nearest 0.1 ) } \\
\hline $0.1 \%$ & 1.0 for all 14 pairs of tanks \\
\hline $0.3 \%$ & 1.0 for all 14 pairs of tanks \\
\hline $1.0 \%$ & $0.3,0.8,0.5,1,1,1,1,1,1,1,0.4,1,1,1$ \\
\hline $5.0 \%$ & 0 except tank $8: 1$, tank $12: 0.9$ \\
\hline $\mathrm{V}_{8 \mathrm{~kg}}$ & 0 for all 14 pairs of tanks \\
\hline
\end{tabular}

Recall that in the previous section we considered the protracted-loss cases over 1 year, so we do not present any further protracted-loss cases here.

\subsection{Analyses: summary and conclusions}

We present in Table 12 a qualitative summary of the loss detection probability $(1-\beta)$ for a system-wide (15-tank system) false-alarm probability of 0.05 for volume or mass loss sufficient to divert $8 \mathrm{~kg} \mathrm{Pu}$ (with or without bias corrections). Our results are conservative (we tend to underestimate the loss-detection probability) because of the conservative method (Bonferroni) used to correct the individual $\alpha$ 's for multiple testing.

Table 12. Loss Detection Probabilities.

\begin{tabular}{|c|c|c|c|}
\hline $\begin{array}{c}\text { Abrupt diversion } \\
\text { during wait mode }\end{array}$ & $\begin{array}{c}\text { Abrupt diversion } \\
\text { during transfer mode }\end{array}$ & $\begin{array}{c}\text { Protracted diversion } \\
\text { during wait mode }\end{array}$ & $\begin{array}{c}\text { Protracted diversion } \\
\text { during transfer mode }\end{array}$ \\
\hline Very High & Sufficiently high & Very low & Extremely low \\
\hline
\end{tabular}

Because bias corrections are feasible via SM data, this paper has considered the impact of bias corrections data both on worst-case protracted loss detection and on abrupt loss detection. For a three-tank system with all B/B mode tanks, there could be very substantial improvement in $\beta$. However, for a 15 -tank system with some $\mathrm{B} / \mathrm{C}$ or $\mathrm{C} / \mathrm{B}$ mode tanks, we expect only very modest or no improvement in $\beta$, largely because of the transfer modes and specifically due to increased error variance in estimating shipments or receipts when one of the tanks has a $\mathrm{C}$ mode. The same conclusion would hold if we also measured Pu concentration in all 15 tanks. We attempted to quantify the likely impact of bias corrections, although the actual bias corrections could perform better or worse than they did in any of the cases presented. Despite the anticipated very modest to zero improvement in the worst-case protracted-loss detection ability, we believe that SM will greatly enhance safeguards with minimal effort. For reasons mentioned elsewhere (such as the ability to validate error models and the ability to implement anomaly resolution [6], [7] ) plus the anticipated very good abrupt-loss detection illustrated in this paper, we are very strongly in favor of including 
SM in modern safeguards approaches. The implementation of intelligent software to find, classify, and interpret all key tank events is the most critical need today.

Finally, we point out that the simulated diversions with replacement (of incorrect density solution, so mass changes but volume does not) impact the diversion tank and all downstream tanks. Therefore, more comprehensive multivariate diagnostics [9] could be a worthy addition to our simple univariate and multivariate tests described here. Some of these comprehensive multivariate diagnostics have been implemented and tested for a simple three-tank system [10], and results are promising, but there is more effort involved.

\section{Summary and Conclusions}

This final report for the Simulation and Analysis of Reprocessing Plant Data task has presented considerable detail on both the simulation and the analysis of SM data. An existing simulation capability (FacSim) was extended to model solution density using empirical relations between density and constituent concentrations. Under a previous task described in Ref. 3, prototype software to find and classify all key tank events was written, which was applied to SM data that was simulated using the extended version of FacSim. Small but reasonable errors (both random and systematic) were added. Then, decision thresholds were set to monitor both for abrupt and protracted diversion for a system-wide, yearly false alarm probability of 0.05 . Basic properties of the normal (gaussian) distribution could then be used to estimate the loss detection probabilities reported $(1-\beta)$, which are expected to be good for abrupt diversion and poor for protracted diversion. Because of reasons mentioned elsewhere (such as the ability to validate error models and the ability to implement anomaly resolution [6], [7]) plus the anticipated very good abrupt-loss detection illustrated in this paper, we are very strongly in favor of including SM in modern safeguards approaches. The implementation of intelligent software to find, classify, and interpret all key tank events is the most critical need for analysis of SM data today.

\section{References}

[1] T. K. Li et al., "Near-Real-Time Accounting (NRTA) as a Safeguards Measure at the Rokkasho Reprocessing Plant," Los Alamos National Laboratory, Safeguards Science and Technology Group technical report NIS-5-96-1 (September 1996).

[2] W. S. Groenier, "Technical Manual for SEPHIS MOD4, Version 2.11," Oak Ridge National Laboratory technical report ORNL/TM-11589 (1991).

[3] Tom Burr and Larry Wangen, "Development and Evaluation of Methods for Safeguards use of Solution Monitoring Data," Los Alamos National Laboratory report LA-13185-MS (ISPO389) (September 1996). 
[4] F. Franssen, "Tank Data Acquisition and Evaluation in a Large Scale Reprocessing Plant," Proc. 17th Annual ESARDA Symposium on Safeguards and Nuclear Material Management, Aachen (Aix-la-Chapelle), Germany, 1995 (Joint Research Centre, Ispra, Italy, 1995).

[5] R. Avenhaus and J. Jaech, "On Subdividing Material Balances in Time and/or Space," Inst. Nucl. Mater. Manage. 10 3, 24-33 (1981).

[6] Tom Burr and Larry Wangen, "Enhanced Safeguards Via Solution Monitoring, Los Alamos National Laboratory report LA-13186-MS (ISPO-390) (September 1996).

[7] Tom L. Burr, C. Alton Coulter, and Lawrence E. Wangen, "Solution Monitoring: Quantitative Benefits to Safeguards," presented at the IAEA Symposium on International Safeguards, Vienna, Austria, October 13-17, 1997; Los Alamos National Laboratory document LA-UR97-3467 (1997).

[8] R. Johnson, and D. Wichern, Applied Multivariate Analysis (Prentice Hall, 1988).

[9] R. Crosier, "Multivariate Generalizations of Cumulative Sum Quality Control Schemes," Technometrics 30, 291-303 (1988).

[10] T. Burr, J. Jones, and L. Wangen, "Multivariate Diagnostics and Anomaly Detection for Nuclear Safeguards," Nucl. Mater. Manage. XXIII, 1000-1007 (1994). 
Dear Dr. Alton Coulter

Safeguards Systems Group N-4

Los Alamos National Laboratory

P.O.Box 1663

MS E541

Los Alamos

New Mexico 87545

USA

Subject : Use of RRP model for Agency study under POTAS task

Dear Dr. Coulter;

At the request of Mr. Tom Canada; Head of JNFL Project, SGOA, IAEA, I would like to inform you that JNFL provides LANL with the consent to use the RRP model already available for LANL , under the following condition;

1. The model is to be used only for assisting the IAEA to construct the safeguards approach of RRP, under POTAS task D00934.

2. The work scope to be carried out using the RRP model under the task D00934 shall obtain authorization by Mr. Tom Canada; Head of JNFL Project SGOA of IAEA.

3. The main objective to use the RRP model is assumed to assist the Agency to construct the safeguards approach of RRP, but not for R \& D type works to be published. Hence, if it happens, a written consent of JNFL and IAEA is necessary.

I wish you to continue our cooperation for improving RRP safeguards.

CC. T.Canada/IAEA

Yours sincerely;

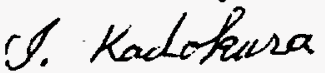

Itsuo Kadokura

Manager; Safeguards Planning Div.

Safeguards Department

JNFL

Fukokuseimei Bldg.

2-2, 2-chome, Uchisaiwai-cho

Chiyoda-ku, Tokyo 100 Japan

H.Kuroi/JNFL 